University of Rhode Island

DigitalCommons@URI

Open Access Dissertations

2017

\title{
The Influence of Interfacial Effects by PVDF on the Fluorescent Properties of Rhodamine 6G
}

\author{
Mona Abdulmuti Alhasani \\ University of Rhode Island, mona_alhasani@my.uri.edu
}

Follow this and additional works at: https://digitalcommons.uri.edu/oa_diss

\section{Recommended Citation}

Alhasani, Mona Abdulmuti, "The Influence of Interfacial Effects by PVDF on the Fluorescent Properties of Rhodamine 6G" (2017). Open Access Dissertations. Paper 612.

https://digitalcommons.uri.edu/oa_diss/612

This Dissertation is brought to you for free and open access by DigitalCommons@URI. It has been accepted for inclusion in Open Access Dissertations by an authorized administrator of DigitalCommons@URI. For more information, please contact digitalcommons-group@uri.edu. 
THE INFLUENCE OF INTERFACIAL EFFECTS BY PVDF ON THE FLUORESCENT PROPERTIES OF RHODAMINE 6G BY MONA ABDULMUTI ALHASANI

A DISSERTATION SUBMITTED IN PARTIAL FULFILLMENT OF THE REQUIREMENTS FOR THE DEGREE OF DOCTOR OF PHILOSOPHY IN CHEMISTRY

UNIVERSITY OF RHODE ISLAND 2017 
DOCTOR OF PHILOSOPHY DISSERTATION

OF

MONA ABDULMUTI ALHASANI

APPROVED:

Dissertation Committee:

Major Professor William B. Euler

Louis J. Kirschenbaum

Geoffrey D. Bothun

Nasser H. Zawia

DEAN OF THE GRADUATE SCHOOL

UNIVERSITY OF RHODE ISLAND

2017 


\begin{abstract}
In Chapter 1, Fluorescent enhancements have been achieved using a simple layered structure: fluorophore, polymer/metal ion, glass substrate. The polymer/metal ion layer apparently has a strong influence on the emission response of the fluorophore by removing the dye aggregation. This data supports that the addition of higher concentration of hydrated transition metal salt increases the production of $\beta$-phase in the Polyvinylidene Difluoride (PVDF). The absorption spectra intensity increased as the amount of $\mathrm{Zn}^{2+}$ is increased in the substrate while the Rhodamine 6G (Rh6G) thickness is kept constant. Investigation into the means of $\beta$-phase production and the influence of the interfacial region effect on the fluorescence enhancement was completed and reported in this work. The goal of this study is to understand the interfacial properties that control the nature of the fluorescent emission and determine the structure of the fluorophore on different substrates.
\end{abstract}

Chapter 2 of this dissertation shows an investigation of the role of the polymer substrate, the solvent and the reaction between $\mathrm{Rh} 6 \mathrm{G}$ and $\mathrm{Zn}^{2+}$. Studying the reaction between $\mathrm{Rh} 6 \mathrm{G}$ and $\mathrm{Zn}^{2+}$ was done on glass slides in the absence of polymer. In order to study the role of the solvent, films of PVDF doped with $\mathrm{Zn}^{2+}$ were cast from pure acetone. PVDF polymer was replaced by PMMA to evaluate the role of the polymer in the films.

The response of emission signal of TNT, as a function of Rhodamine $6 \mathrm{G}$ concentration and mol \% of $\mathrm{Zn}^{2+}$ has been studied in Chapter 3. Using a different concentration of Rhodamine $6 \mathrm{G}$ and mol $\%$ of metal ion, the signal is quenched by a 
notably large amount. Different analytes can be applied and emission signals can be collected to find a pattern that could be used to identify the explosives. 


\section{ACKNOWLEDGMENTS}

The path toward this dissertation has been long and full of obstacles and difficulties. My goal of being a PhD student over seas starts since my first year as an undergrad student at Saudi Arabia. I graduated and got a scholarship to study abroad for a master degree. I stared my master degree at 2011 having my first son at first week of classes! Two years later I walked in my graduation ceremony 9 months pregnant with my second son!! However, I had a high GPA that allowed me to extend my scholarship for a $\mathrm{PhD}$.

The completion of this dissertation is a thankful to the special people around me. I would like to take this opportunity to thank them all.

First, thanks god for blessing me with a loving and caring family, husband, kids and friends. Thanks god for giving me such a great opportunity to success in my school. Thanks for making my dream true and offering me the job that I always want. Thanks for giving me the strength to overcome all the difficulties I had for the past seven years, starting from being away from my family, friends and my great homeland. Saudi Arabia is the homeland that gives me everything. It is enough to say that it gives me the chance to be who I am today and guarantees my future.

Second, I would like to thank my amazing professor Dr. Euler the chair of the chemistry department for all the wonderful lessons that he has taught me during the last 4 years of my $\mathrm{PhD}$. Thank you for helping me making my dream a reality! He is truly the most remarkable professor I have ever had in my academic journey! And he will always be the best example for me. 
Third, I would like to thank Dr. Dhia Habboush and Dr. Eid Alkhatib from Sacred Heart University at Connecticut for their support and courage to join the Chemistry Department at URI; it is so competitive at URI especially when working with Dr. Euler. I had the pleasure to be academically supervised by Dr. Euler as I was academically supervised by Dr. Dhia Habboush.

Last, thanks to my wonderful Dad for his support, love and unlimited help. Thanks to my Mom for her unlimited love, support and for believing in me. And I would like to thank my wonderful husband Ahmed and my kids: Faris, Feras and Yousef. You are my soldiers. 


\section{PREFACE}

The following research is presented in manuscript format according to the guidelines provided by the Graduate School of the University of Rhode Island. The dissertation is separated into three chapters.

The first chapter is entitled "The influence of $\mathrm{Zn}^{2+}-$ doped PVDF on the fluorescent properties of Rhodamine $6 \mathrm{G}^{\prime \prime}$ and is in preparation for submission to the Journal of Langmuir (ACS).

The second chapter is entitled "The fluorescent spectroscopy of $\mathrm{Zn}^{2+}-$ doped PMMA of Rhodamine 6G" and is in preparation for submission to the Journal of Langmuir (ACS).

The third chapter is entitled "Detection of gas-phase explosive analytes using fluorescent spectroscopy of PVDF doped with Rhodamine 6G" and is in preparation for submission to the Journal of Langmuir (ACS). 


\section{TABLE OF CONTENTS}

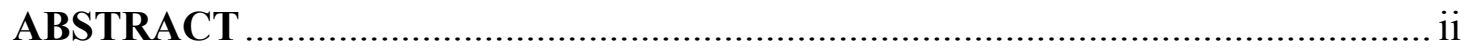

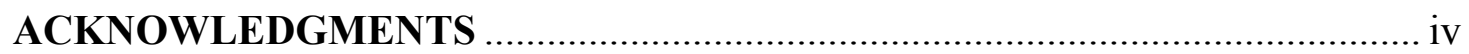

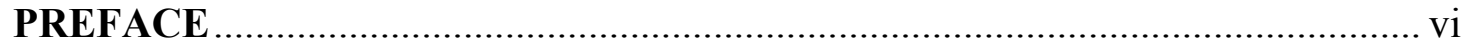

TABLE OF CONTENTS ......................................................................... vii

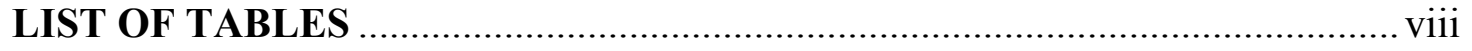

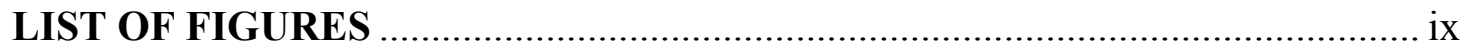

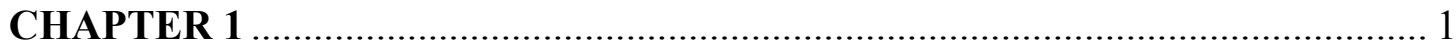

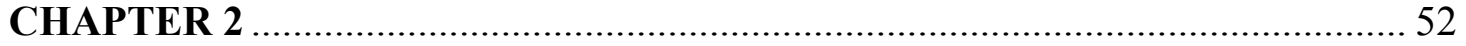

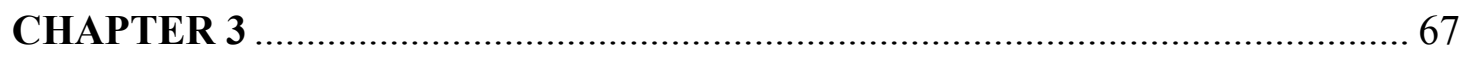

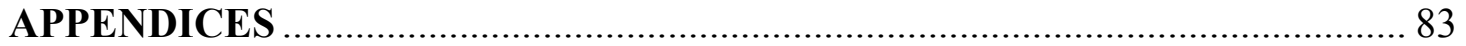




\section{LIST OF TABLES}

\section{Chapter 1:}

TABle 1. Deconvoluted Gaussian PeAKS For the absorbance SPeCtra. PeaK POSITION $\left(\Lambda_{\text {MAX }}\right)$ AND FWHM $(\Gamma)$ HAVE AN UNCERTAINTY OF \pm 2 NM. .................. 13

TABLE 2. MEASURED EXCITED STATE LIFE TIMES FOR RH6G THIN FILMS ON $\mathrm{ZN}^{2+}$-DOPED

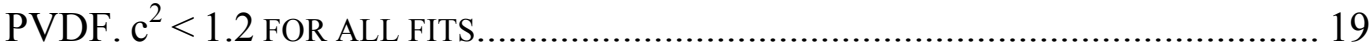

TABLE 3. SUMMARY OF EFFECTS ON RH6G BY $\mathrm{ZN}^{2+}$-DOPED PVDF. THE DATA FOR NO $\mathrm{ZN}^{2+}$ ARE TAKEN FROM REFERENCE 33

\section{Chapter 2:}

TABLE 1. THE RESPONSE OF RH6G ON DIFFERENT SUBSTRATES. 64 


\section{LIST OF FIGURES}

\section{Chapter 1:}

FIGURE 1. (A) INFRARED SPECTRA OF PVDF FILMS WITH THE INDICATED MOLE PERCENT OF ZINC (II) IN THE FINGERPRINT REGION. THE REPRESENTATIVE PHASE MARKERS ARE SHOWN AS VERTICAL LINES. (B) INFRARED SPECTRA OF PVDF FILMS WITH THE INDICATED MOLE PERCENT OF ZINC (II) IN THE CARBONYL REGION. THE VERTICAL LINES SHOW THE POSITION OF THE CARBONYL STRETCH FOR FREE ACETONE AND FREE DMF. ALL SPECTRA ARE NORMALIZED TO ACCOUNT FOR THICKNESS USING THE PEAK AT $1170 \mathrm{CM}^{-1}$ (REFERENCE 38).

FIGURE 2. (A) TGA MASS LOSS CURVES FOR DIFFERENT SAMPLES OF PVDF DOPED WITH $\mathrm{ZN}^{2+}$. (B) TOTAL MASS LOSS AS A FUNCTION OF MOLE $\% \mathrm{ZN}^{2+}$. THE SOLID LINE IS THE FIT FOR 6 DMF AND $6 \mathrm{H}_{2} \mathrm{O}$ MOLECULES PER $\mathrm{ZN}^{2+}$ AND 0.08 DMF PER $\mathrm{CH}_{2} \mathrm{CF}_{2}$ REPEAT UNIT.

FigURE 3. LEFT: AFM IMAGES OF PVDF FILMS WITH DIFFERENT LEVELS OF $\mathrm{ZN}^{2+}$ DOPED INTO THE FILM. ALL OF THE FILMS ARE $475 \pm 75$ NM THICK. RigHT: SURFACE ROUGHNESS AS A FUNCTION $\mathrm{ZN}^{2+}$ DOPING LEVEL...

FIGURE 4. ABSORBANCE SPECTRA AS A FUNCTION OF RH6G CONCENTRATION USED IN SPIN-CASTING. A: CAST ONTO PVDF/ $1 \mathrm{MOL} \% \mathrm{ZN}^{2+}$; B: CAST ONTO PVDF $/ 3 \mathrm{MOL} \%$ $\mathrm{ZN}^{2+}$; C: CAST ONTO PVDF/5 MOL $\% \mathrm{ZN}^{2+}$; D: ABSORBANCE AT $1_{\text {MAX VS. SPIN- }}$ CASTING CONCENTRATION FOR DIFFERENT $\mathrm{ZN}^{2+}$ DOPING LEVELS. NOTE THAT THE ABSORBANCE SCALES ARE THE SAME FOR A, B, AND C.

FIGURE 5. ABSORBANCE SPECTRA OF RH6G ON PVDF SURFACES WITH DIFFERENT ZN ${ }^{2+}$ DOPING LEVELS. A: $1 \times 10^{-4} \mathrm{M}$ RH6G CAST ONTO $\mathrm{ZN}^{2+}$ DOPED PVDF; B: $5 \times 10^{-4} \mathrm{M}$ RH6G CAST ONTO ZN ${ }^{2+}$ DOPED PVDF; C: $9 \times 10^{-4} \mathrm{M}$ RH6G CAST ONTO $\mathrm{ZN}^{2+}$ DOPED PVDF; D: ABSORBANCE AT $1_{\text {MAX }}$ ON $Z_{N^{2+}}$ DOPED PVDF VS. FOR DIFFERENT RH6G SPIN-CASTING CONCENTRATION. THE LINES SHOW THE LINEAR RELATIONSHIP FOR THREE OF THE CONCENTRATIONS, $1 \times 10^{-4} \mathrm{M}, 5 \times 10^{-4} \mathrm{M}$, AND $9 \times 10^{-4} \mathrm{M}$ (OTHERS ARE NOT SHOWN FOR THE SAKE OF CLARITY). NOTE THAT THE ABSORBANCE SCALES ARE THE SAME FOR A, B, AND C.

FIGURE 6. EMISSION SPECTRA AS A FUNCTION OF RH6G CONCENTRATION USED IN SPINCASTING. A: CAST ONTO PVDF/ $1 \mathrm{MOL} \% \mathrm{ZN}^{2+}$; B: CAST ONTO PVDF $/ 3 \mathrm{MOL} \%$ $\mathrm{ZN}^{2+}$; C: CAST ONTO PVDF $/ 5 \mathrm{MOL} \% \mathrm{ZN}^{2+}$; D: $1_{\mathrm{MAX}}$ VS. SPIN-CASTING CONCENTRATION FOR DIFFERENT $Z_{\mathrm{N}^{2+}}$ DOPING LEVELS. NOTE THAT THE INTENSITY SCALES ARE THE SAME FOR A, B, AND C. 14 
FIGURE 7. EMISSION SPECTRA OF RH6G ON PVDF SURFACES WITH DIFFERENT $\mathrm{ZN}^{2+}$ DOPING LEVELS. A: $1 \times 10^{-4} \mathrm{M}$ RH6G CAST ONTO $\mathrm{ZN}^{2+}$ DOPED PVDF; B: $5 \times 10^{-4} \mathrm{M}$ RH6G CAST ONTO ZN ${ }^{2+}$ DOPED PVDF; C: $9 \times 10^{-4}$ M RH6G CAST ONTO $\mathrm{ZN}^{2+}$ DOPED PVDF; D: $1_{\text {MAX }}$ ON Z $\mathrm{N}^{2+}$-DOPED PVDF VS. FOR DIFFERENT RH6G SPIN-CASTING CONCENTRATION. NOTE THAT THE INTENSITY SCALES ARE THE SAME FOR A, B, AND C.

FiguRE 8. TOTAL AREA UNDER THE EMISSION SPECTRA AS A FUNCTION OF: A [RH6G] USED IN SPIN-CASTING AND B Z $\mathrm{N}^{2+}$ DOPING LEVEL IN THE PVDF FILM.

FIGURE 9. WAVELENGTH MAXIMA FOUND FROM DECONVOLUTION OF THE EMISSION SPECTRA. ERRORS IN EACH DATA POINT ARE \pm 3 NM.

Figure 10. NoRMALIZED EXCITATION (SOLID LINES, DETECTION DONE AT $1_{\text {MAX }}$ OF THE EMISSION SPECTRUM), ABSORBANCE (DASHED LINES), AND EMISSION (DOTTED LINES) SPECTRA FOR RH6G ON ZN ${ }^{2+}$-DOPED PVDF. A: 1 MOL $\% \mathrm{ZN}^{2+}$ IN PVDF. B: $5 \mathrm{MOL} \% \mathrm{ZN}^{2+}$ IN PVDF. FOR BOTH FIGURES: BLACK LINES - [RH6G] USED IN SPINCASTING $=1 \times 10^{-4} \mathrm{M}$; RED LINES $-[$ RH6G $]$ USED IN SPIN-CASTING $=9 \times 10^{-4} \mathrm{M} \ldots 17$

FiguRE 11. EXCITED STATE DECAYS FOR RH6G ON ZN ${ }^{2+}$-DOPED PVDF. IRF $=$ INSTRUMENT RESPONSE FUNCTION.

\section{Chapter 2:}

Figure 1. AbSORBAnCE Spectra of $9 \times 10-4$ M Rh6G Doped With $\mathrm{ZN}^{2+}$ on Glass

SLIDE. 58

FIGURE 2. ABSORBANCE SPECTRA OF 1X10-4 M RH6G ON PVDF THIN FILMS CAST FROM ACETONE AS A FUNCTION OF $\mathrm{ZN}^{2+}$ MOL $\%$. 59

FIGURE 3. ABSORBANCE SPECTRA OF 9x10-4 M RH6G ON PVDF THIN FILMS CAST FROM ACETONE AS A FUNCTION OF $\mathrm{ZN}^{2+}$ MOL $\%$.

FigURE 4. POLARIZATION SPECTRA OF 9X10-4M RH6G ON PVDF DOPED WITH 5 MOL\% $\mathrm{ZN}^{2+}$ 60

FIGURE 5. ABSORBANCE SPECTRA OF 9x10-4M RH6G ON PMMA/ZN ${ }^{2+}$ THIN FILMS AS A FUNCTION OF $\mathrm{ZN}^{2+} \mathrm{MOL} \%$.

FIGURE 6. EMISSION SPECTRA OF [RH6G] ON PVDF IN ACETONE THIN FILMS AS A FUNCTION OF $\mathrm{ZN}^{2+}$ MOL $\%$..

FIGURE 7. EMISSION SPECTRA OF [RH6G] ON PMMA THIN FILMS AS A FUNCTION OF $\mathrm{ZN}^{2+} \mathrm{MOL} \%$. 


\section{Chapter 3:}

FIGURE 1. EMISSION SPECTRA OF DIFFERENT CONCENTRATIONS OF RH6G ON PVDF SURFACES WITH DIFFERENT $\mathrm{ZN}^{2+}$ DOPING LEVELS AFTER EXPOSURE TO TNT........ 75 


\title{
CHAPTER 1
}

\section{The Influence of $\mathrm{Zn}^{2+}-$ Doped PVDF on the Fluorescent Properties of Rhodamine 6G}

\author{
Mona Alhasani, ${ }^{1}$ Anju Gupta, ${ }^{2}$ and William B. Euler ${ }^{1} *$ \\ 1. Department of Chemistry, University of Rhode Island, 140 Flagg Road, Kingston, \\ RI 02881
}

2. Department of Chemical Engineering, Rochester Institute of Technology, Rochester, NY

* Corresponding Authors weuler@chm.uri.edu, phone 401-874-5090, fax 401-8745072; argche@rit.edu, phone 585-475-4093, fax 585-475-4450

The following is submitted to the Journal of Langmuir (ACS), and is presented here in manuscript format. 


\section{ABSTRACT}

The photophysical properties of rhodamine $6 \mathrm{G}(\mathrm{Rh} 6 \mathrm{G})$ deposited on the surface of $\mathrm{Zn}^{2+}$-doped polyvinylidene difluoride (PVDF) are reported. The zinc ion induces an increased amount of the ferroelectric $\beta$-phase in the PVDF. This, in turn, prevents aggregation of the Rh6G on the surface. Absorption spectra lack the low energy signature of Rh6G aggregates even for the thickest films. The absorption spectra also show an unusual increase in intensity as the amount of $\mathrm{Zn}^{2+}$ is increased in the substrate while the Rh6G thickness is kept constant. The excitation spectra match the absorption spectra. As the $\mathrm{Zn}^{2+}$ ion concentration increases in the PVDF the emission spectra maxima shift to lower energy and the intensity of the emission increases. It is proposed that exciton diffusion in the Rh6G layer accounts for the experimental observations. 


\section{INTRODUCTION}

We have been investigating surface properties of fluorescent dyes since they have been shown to be effective sensors for a number of analytes. ${ }^{1-15}$ We were able to demonstrate that the emission intensity can be significantly increased, up to three orders of magnitude, using a three layer structure where a transparent polymer was sandwiched between the fluorophore and the substrate ${ }^{11}$, but the origin of this enhancement was unclear. Fluorescent spectroscopy is often used to study surface properties. ${ }^{16-22}$ So, as a representative example we used rhodamine 6G (Rh6G), whose properties have been heavily studied, ${ }^{23-31}$ as a surface probe on a variety of substrate structures to determine the cause for the emission enhancement. When Rh6G is deposited as a single layer on a glass surface the fluorescent properties are strongly thickness dependent. ${ }^{32}$ For thin films, submonolayer thickness, the emission is dominated by monomers of Rh6G. However, as the thickness increases both excimer and exciton emission could be detected. When the film is several nm thick, representing more than three layers of $\mathrm{Rh} 6 \mathrm{G}$, the emission intensity is significantly quenched and arises primarily from excitons. When a layer of poly(vinylidene difluoride) (PVDF) is placed between the glass substrate and the Rh6G surface the overall emission is enhanced. ${ }^{11,33}$ Three components contributed to the enhancement. First, the PVDF surface was rough, leading to deposition of more fluorophores per unit area on the polymer. Second, the polymer layer allowed internal reflection of the incident light beam, which led to increased absorption and subsequent emission. Finally, the polarity of the polymer may have contributed to the increased emission. However, structurally the $\mathrm{Rh} 6 \mathrm{G}$ followed the same pattern as on glass: at low 
thicknesses monomers were dominant and at high thicknesses aggregation determined the photophysical properties.

PVDF is an interesting polymer because it can be found primarily in three different phases: the nonpolar $\alpha$-phase, the polar $\gamma$-phase, and the ferroelectric $\beta$ phase. ${ }^{34-43}$ Casting films of PVDF into uniform phases is challenging - normally a mixture of $\alpha$ - and $\gamma$-phases is found from typical spin-casting conditions. ${ }^{38} \mathrm{We}$ recently reported that doping PVDF with a transition metal salt could induce $\beta$-phase formation. ${ }^{44}$ In that work $\mathrm{Co}^{2+}$ was used as the doping agent and we showed that the complexation of the metal ion to the F atoms in the PVDF induced a cooperative effect to increase the amount of $\beta$-phase. Thin films of the $\mathrm{Co}^{2+}$-doped PVDF behaved as relaxor ferroelectrics, indicating that the electrical properties of the films were controlled by the $\beta$-phase composition.

In this work we use $\mathrm{Zn}^{2+}$ as a dopant to control the phase behavior of PVDF in order to study the effects on Rh6G at the polymer/fluorophore interface. We chose zinc(II) over cobalt(II) to remove the complications of the d-d transitions in the visible absorption spectrum and to eliminate any effects that might be induced by the magnetic moment of $\mathrm{Co}^{2+}$. As expected, the zinc ion induced $\beta$-phase formation. However, the presence of the metal ion also completely suppressed aggregation of Rh6G on the polymer surface, even at a doping level as low as $1 \mathrm{~mol} \% \mathrm{Zn}^{2+}$. 


\section{EXPERIMENTAL}

Polyvinylidene fluoride with $\mathrm{M}_{\mathrm{w}}=534,000 \mathrm{~g} / \mathrm{mol}$ was purchased from Sigma Aldrich. Zinc (II) nitrate hexahydrate, spectral grade acetone, and $\mathrm{N}, \mathrm{N}-$ dimethylformamide (DMF) solvent were purchased from Fisher Scientific. Glass slides were used as a substrate for film formation. The glass slides were cut into dimensions of $3.75 \mathrm{~cm} \times 1.75 \mathrm{~cm}$. The slides were placed in a container of $95 \%$ ethanol (EtOH) and sonicated for 15 minutes. Then they were rinsed three times and placed in distilled water and sonicated for an additional 15 minutes, then dried with $\mathrm{N}_{2}$ gas. ${ }^{13}$ A solvent system of $90 / 10 \mathrm{v} / \mathrm{v}$ of acetone to DMF was used to dissolve the PVDF at a $3 \% \mathrm{w} / \mathrm{v}$ ratio. ${ }^{6,14}$ The polymer solution was placed in a Branson 3510 ultrasonic cleaning device for 3 hours at $40{ }^{\circ} \mathrm{C}$ to ensure that all of the polymer dissolved. Specified mole percentages of zinc(II) determined the mass of $\mathrm{Zn}\left(\mathrm{NO}_{3}\right)_{2} \cdot 6 \mathrm{H}_{2} \mathrm{O}$ that was added to the polymer solution after the sonication process, using a mechanical swirling technique to dissolve the salt followed by an additional 2 to 3 minutes sonication time until the salt was fully dissolved in solution. Nine different $\mathrm{Zn}^{2+}$ mole percentages ranging from 1 to $5 \mathrm{~mol} \%$ were prepared. A series of Rh6G concentrations ranging from $1 \times 10^{-4} \mathrm{M}$ to $9 \times 10^{-4} \mathrm{M}$ were used.

A Laurell Technologies WS-400B-6NPP/LITE spin-coater was used to prepare films on the glass slides. $300 \mu \mathrm{L}$ of polymer solution was placed on the substrate and then spun at $1200 \mathrm{rpm}$ for $45 \mathrm{~s}$ with an acceleration of $1080 \mathrm{~s}^{-2}$. After spin casting of polymer solutions was complete, the samples were then placed in an oven set to $60{ }^{\circ} \mathrm{C}$ for 2 min to dry. The Rh6G solutions were also spun-cast in a similar manner. A 
volume of $50 \mu \mathrm{L}$ was placed on the polymer surface and spun at $1200 \mathrm{rpm}$ for $45 \mathrm{~s}$ with an acceleration of $1080 \mathrm{~s}^{-2}$.

A Perkin-Elmer Lambda 1050 spectrometer was employed for obtaining UVVis spectra. Before the absorbance spectrums were collected, a blank of a clean glass slide was taken. A Horiba (JobinYvon) Fluorolog spectrometer was used for steadystate fluorescence measurements and data were collected at 60 -degree angle relative to the excitation beam. The emission wavelength range was from 520 to $800 \mathrm{~nm}$ with a slit width of $3.0 \mathrm{~nm}$. The excitation wavelength range was from $300 \mathrm{~nm}$ to $800 \mathrm{~nm}$ with a slit width of $2.0 \mathrm{~nm}$ to correspond with the absorbance spectrum. A PerkinElmer Spectrum 100 FTIR spectrometer was used for infrared measurements in ATR mode. Samples were scanned from 650 to $4000 \mathrm{~cm}^{-1}$ at a resolution of $1 \mathrm{~cm}^{-1}$. A Filmetrics F40 microscope was used to measure the film thickness. TGA measurements were done on a TA Instruments Q50 between 25 and $400{ }^{\circ} \mathrm{C}$ with a heating rate of $10{ }^{\circ} \mathrm{C} / \mathrm{min}$. An Agilent Technologies 5500 AFM was used to collect images of the surface using tapping mode. 


\section{RESULTS AND DISCUSSION}

In this study, spin casting is used to deposit the polymer onto the glass substrate and potentially three phases may exist in the thin films of PVDF. FTIR spectroscopy can easily identify the different phases of PVDF, ${ }^{38}$ and Figure 1 shows the changes in the spectra as $\mathrm{Zn}^{2+}$ is added to the PVDF film. The $\alpha$-phase can be identified by a peak at $764 \mathrm{~cm}^{-1}$, the $\beta$-phase by peaks at $840 \mathrm{~cm}^{-1}$ and $1275 \mathrm{~cm}^{-1}$, and the- $\gamma$ phase by peaks at $840 \mathrm{~cm}^{-1}$ and $1233 \mathrm{~cm}^{-1}$. The FTIR spectroscopy showed an increase in the amount of $\beta$-phase and g-phase present and a decrease in the amount of $\alpha$-phase in the thin films as the amount of $\mathrm{Zn}^{2+}$ is increased, as shown in Fig. 1A. In the carbonyl region there is a strong feature that is not associated with PVDF, as shown in Fig. 1B. The increasing intensity around $1650 \mathrm{~cm}^{-1}$ is due to the presence of coordinated DMF, which increases as the amount of $\mathrm{Zn}^{2+}$ increases. However, even in the absence of $\mathrm{Zn}^{2+}$ a small amount of DMF is retained by the PVDF, as indicated by the solid black line in Fig. 1B. Casting films from pure acetone with no DMF removes the carbonyl peaks but there also is a much smaller amount of $\beta$-phase formed. Deconvolution of the spectra in the carbonyl region shows three peaks $(\mathrm{N}=9$ spectra, uncertainties are one standard deviation): $1673( \pm 3) \mathrm{cm}^{-1}$ (occluded DMF), $1653( \pm 6)$ $\mathrm{cm}^{-1}$ (coordinated DMF), and $1642( \pm 4) \mathrm{cm}^{-1}$ (coordinated DMF). The ratio of the areas of the $1653 \mathrm{~cm}^{-1}$ to the $1642 \mathrm{~cm}^{-1}$ peaks is about 2, suggesting 6-coordinate, tetragonally distorted $\left[\mathrm{Zn}(\mathrm{DMF})_{6}\right]^{2+}$. 

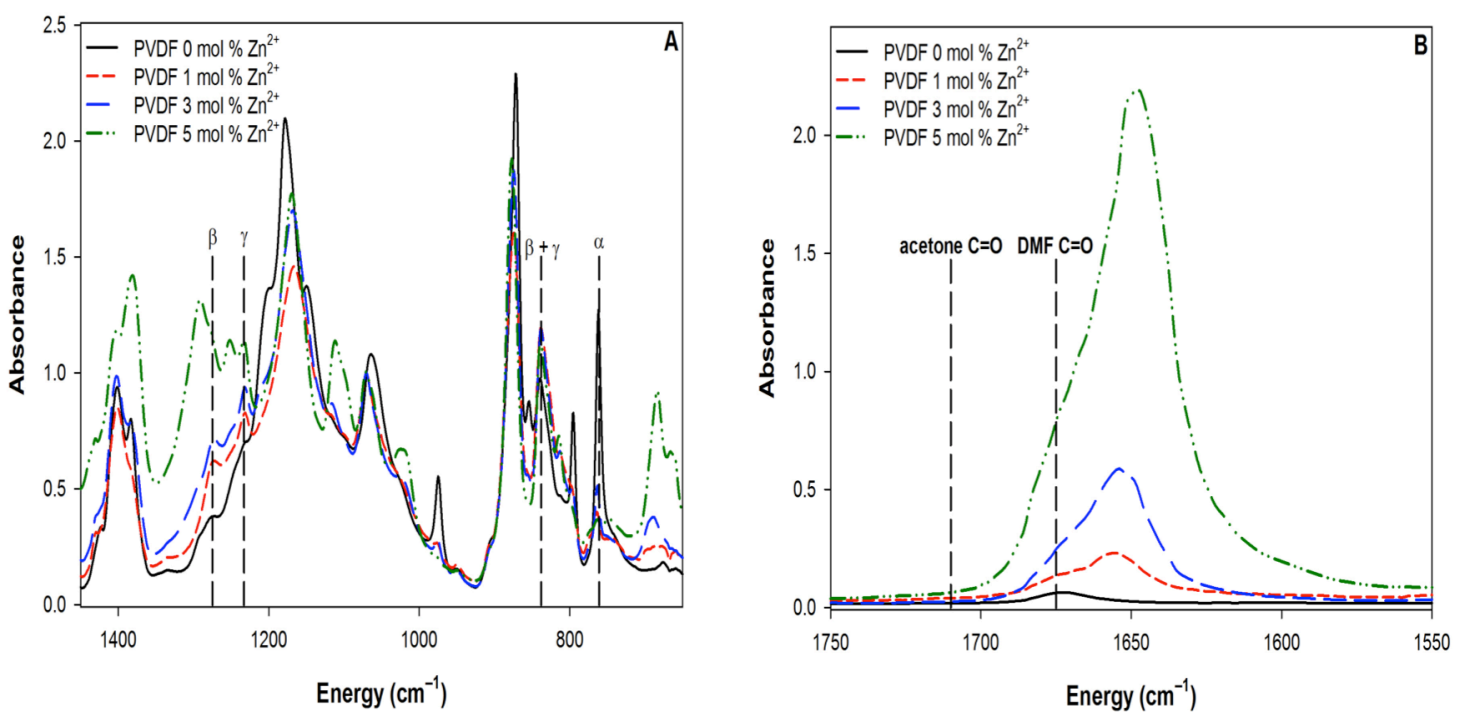

Figure 1. (A) Infrared spectra of PVDF films with the indicated mole percent of zinc (II) in the fingerprint region. The representative phase markers are shown as vertical lines. (B) Infrared spectra of PVDF films with the indicated mole percent of zinc (II) in the carbonyl region. The vertical lines show the position of the carbonyl stretch for free acetone and free DMF. All spectra are normalized to account for thickness using the peak at $1170 \mathrm{~cm}^{-1}$ (reference 38 ).

Thermal Gravimetric Analysis (TGA) was used to help confirm the role of the DMF identified from the FTIR spectroscopy. Figure 2A shows that as the amount of zinc ion is increased there is an increasing mass loss between room temperature and $\sim 230{ }^{\circ} \mathrm{C}$. The broad feature from room temperature to $\sim 180{ }^{\circ} \mathrm{C}$ is assigned to loss of $\mathrm{H}_{2} \mathrm{O}$. The wide temperature range suggests that the water is not coordinated to the metal ion but is trapped in the polymer during spin-casting. The sharper mass loss centered at $210{ }^{\circ} \mathrm{C}$ is assigned to loss of DMF that is coordinated to the $\mathrm{Zn}^{2+}$ ion. The small features above $300{ }^{\circ} \mathrm{C}$ are assigned to decomposition of nitrate ion. Figure $2 \mathrm{~B}$ shows the quantitative fit of the mass loss data. The fit arises from assigning 6 coordinated DMF molecules to each $\mathrm{Zn}^{2+}$ ion, another 0.08 DMF molecules for each repeat unit in the PVDF, and $6 \mathrm{H}_{2} \mathrm{O}$ molecules per $\mathrm{Zn}^{2+}$. This is consistent with the FTIR results, which show a small amount of DMF in the polymer even in the absence 
of zinc(II) ion. The water stoichiometry matches what was used in the sample preparation, the hexahydrate of zinc nitrate.
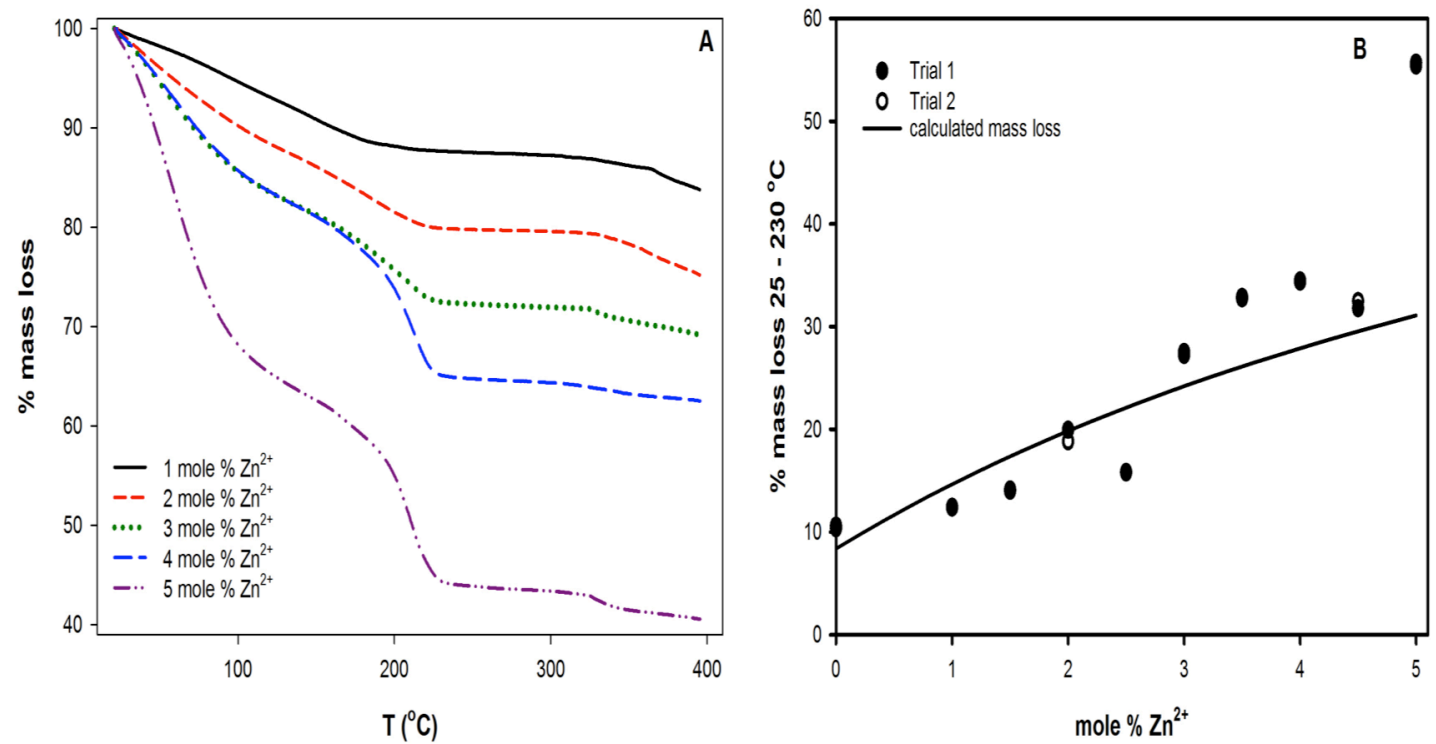

Figure 2. (A) TGA mass loss curves for different samples of PVDF doped with $\mathrm{Zn}^{2+}$. (B) Total mass loss as a function of mole $\% \mathrm{Zn}^{2+}$. The solid line is the fit for $6 \mathrm{DMF}$ and $6 \mathrm{H}_{2} \mathrm{O}$ molecules per $\mathrm{Zn}^{2+}$ and $0.08 \mathrm{DMF}$ per $\mathrm{CH}_{2} \mathrm{CF}_{2}$ repeat unit.

AFM was used to determine the surface characteristics of the different PVDF films. Films were cast under similar conditions with varying amounts of zinc(II) ion to give films of similar thickness, $475 \pm 75 \mathrm{~nm}$. Figure 3 shows the AFM images of these films. The presence of $\mathrm{Zn}^{2+}$ has little effect on the surface properties. All of the films show similarly sized globules and the surface roughness is similar for all films, between 200 and $300 \mathrm{~nm}$, independent of the amount of the metal ion. 

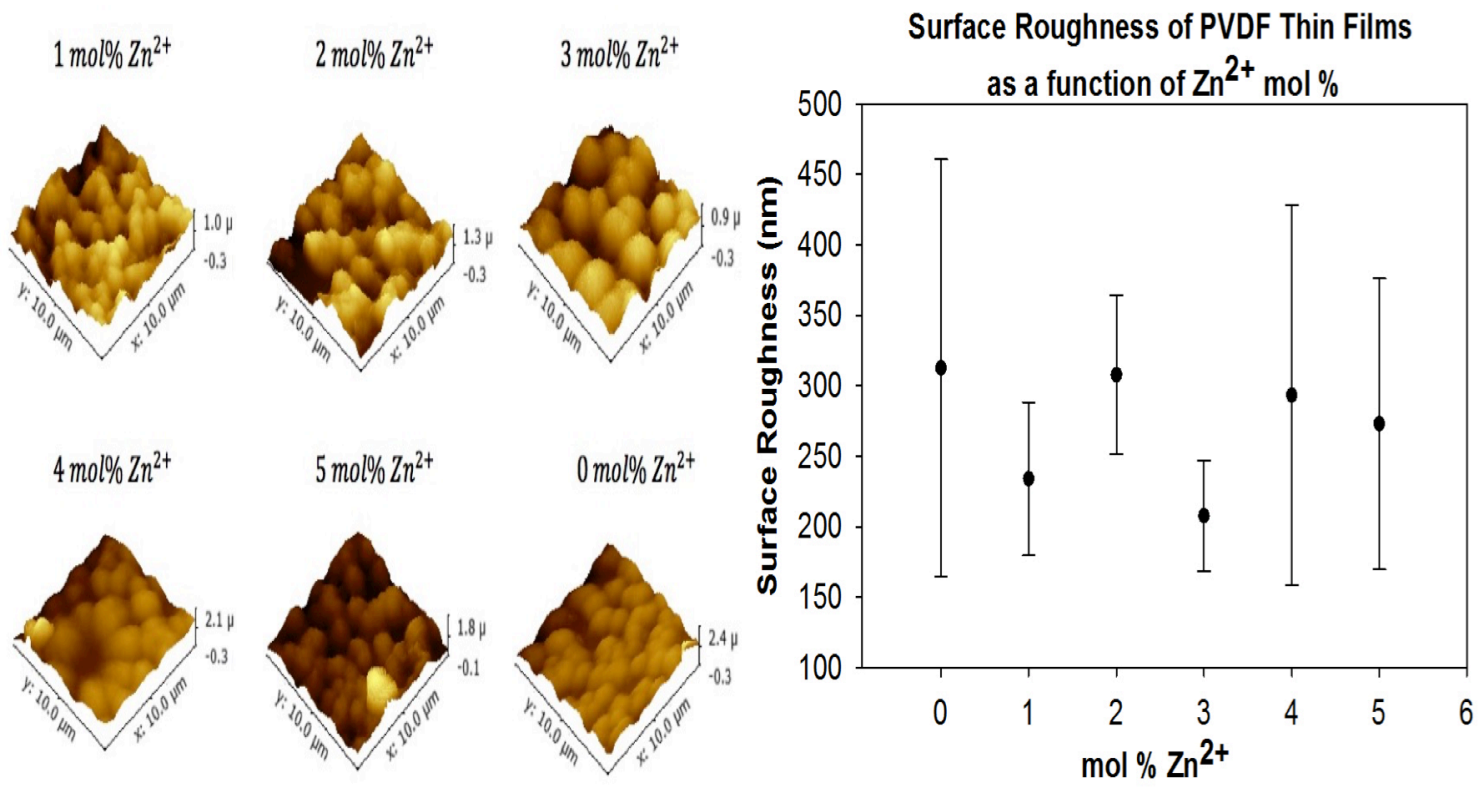

Figure 3. Left: AFM images of PVDF films with different levels of $\mathrm{Zn}^{2+}$ doped into the film. All of the films are $475 \pm 75 \mathrm{~nm}$ thick. Right: Surface roughness as a function $\mathrm{Zn}^{2+}$ doping level.

Figure 4 shows the absorbance spectra of Rh6G on $\mathrm{Zn}^{2+}$-doped PVDF thin films as a function of the concentration of Rh6G used in spin-casting. As the concentration of Rh6G cast onto a PVDF film increases the absorbance increases, as expected as the film gets thicker, shown in Fig. 4D. The spectra shown in Fig. 4A, 4B, and $4 \mathrm{C}$ are all cast with the same concentrations of $\mathrm{Rh} 6 \mathrm{G}$, so presumably are of comparable thicknesses, but as the $\mathrm{Zn}^{2+}$ level in the film increases the absorbance also increases. Since the $\mathrm{Zn}^{2+}$ ion affects the absorbance intensity the nominal thickness of the $\mathrm{Rh} 6 \mathrm{G}$ cannot be determined from the absorbance spectra, as was done previously. ${ }^{32,33}$ Directly measuring the thickness of the Rh6G film by other methods also proved unsuccessful because the surface roughness of the polymer film interferes. As the films get thicker there is no shift in wavelength maximum, in contrast to what 
is observed for Rh6G cast onto glass substrates ${ }^{32}$ and undoped PVDF thin films. ${ }^{33}$ In the absence of $\mathrm{Zn}^{2+}$ the $\mathrm{Rh} 6 \mathrm{G}$ spectra show a gradual shift in $\lambda_{\max }$ from $\sim 520 \mathrm{~nm}$ to $\sim 560 \mathrm{~nm}$ as the $\mathrm{Rh} 6 \mathrm{G}$ gets thicker, which is attributed to aggregation of the dye molecules on the surface. ${ }^{23-33}$ In the presence of the $\mathrm{Zn}^{2+}$, all the spectra have maximum wavelengths $\sim 520 \mathrm{~nm}$. There also is a shoulder at $\sim 495 \mathrm{~nm}$, which is assigned to an exciton. ${ }^{32,33}$ Interaction of the $\mathrm{Zn}^{2+}$ ions with the $\mathrm{Rh} 6 \mathrm{G}$ on the surface is preventing aggregation.
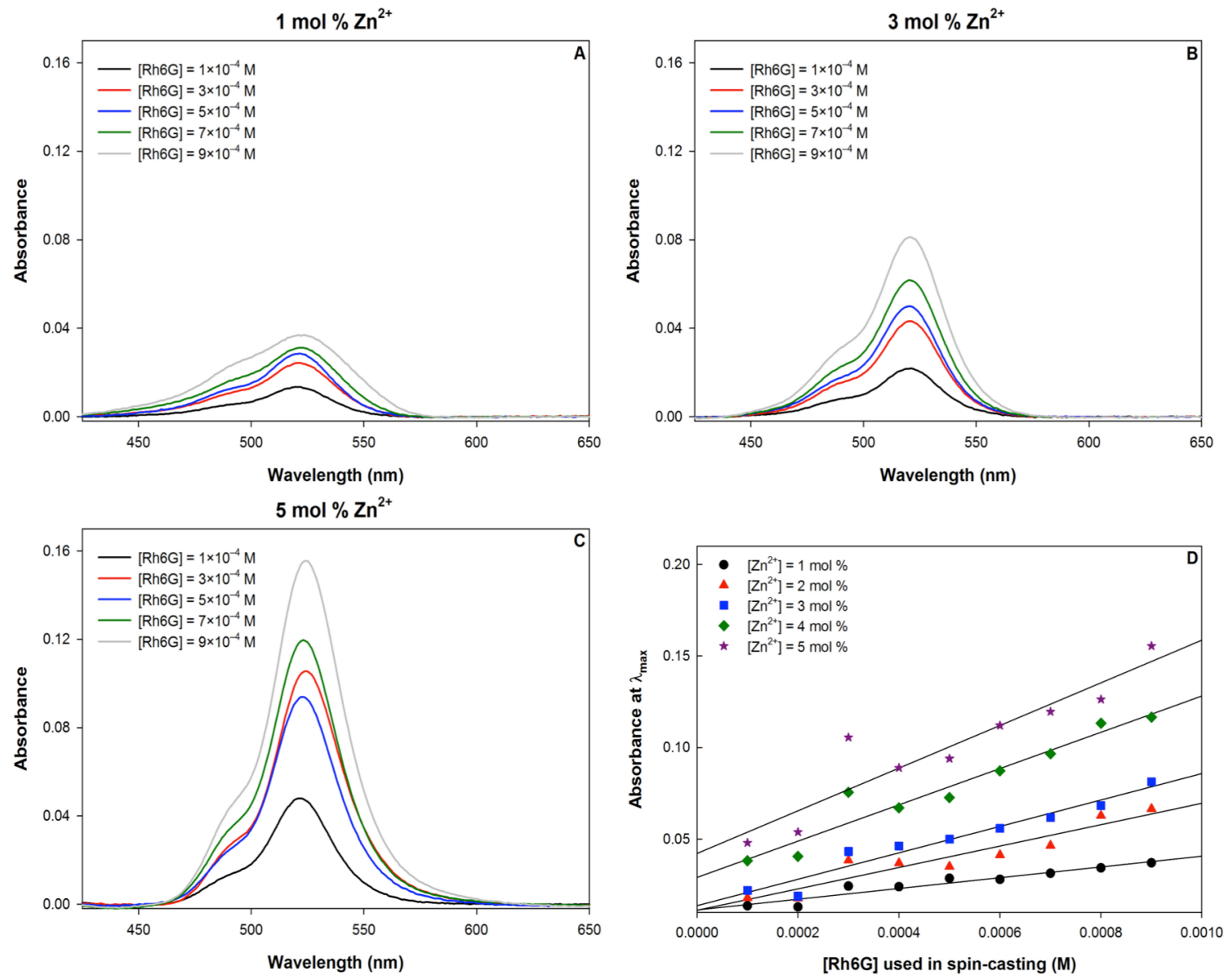

Figure 4. Absorbance spectra as a function of Rh6G concentration used in spincasting. A: cast onto PVDF/ $1 \mathrm{~mol} \% \mathrm{Zn}^{2+}$; B: cast onto PVDF $/ 3 \mathrm{~mol} \% \mathrm{Zn}^{2+}$; C: cast onto $\mathrm{PVDF} / 5 \mathrm{~mol} \% \mathrm{Zn}^{2+}$; $\mathrm{D}$ : absorbance at $1_{\max }$ vs. spin-casting concentration for different $\mathrm{Zn}^{2+}$ doping levels. Note that the absorbance scales are the same for $\mathrm{A}, \mathrm{B}$, and $\mathrm{C}$. 

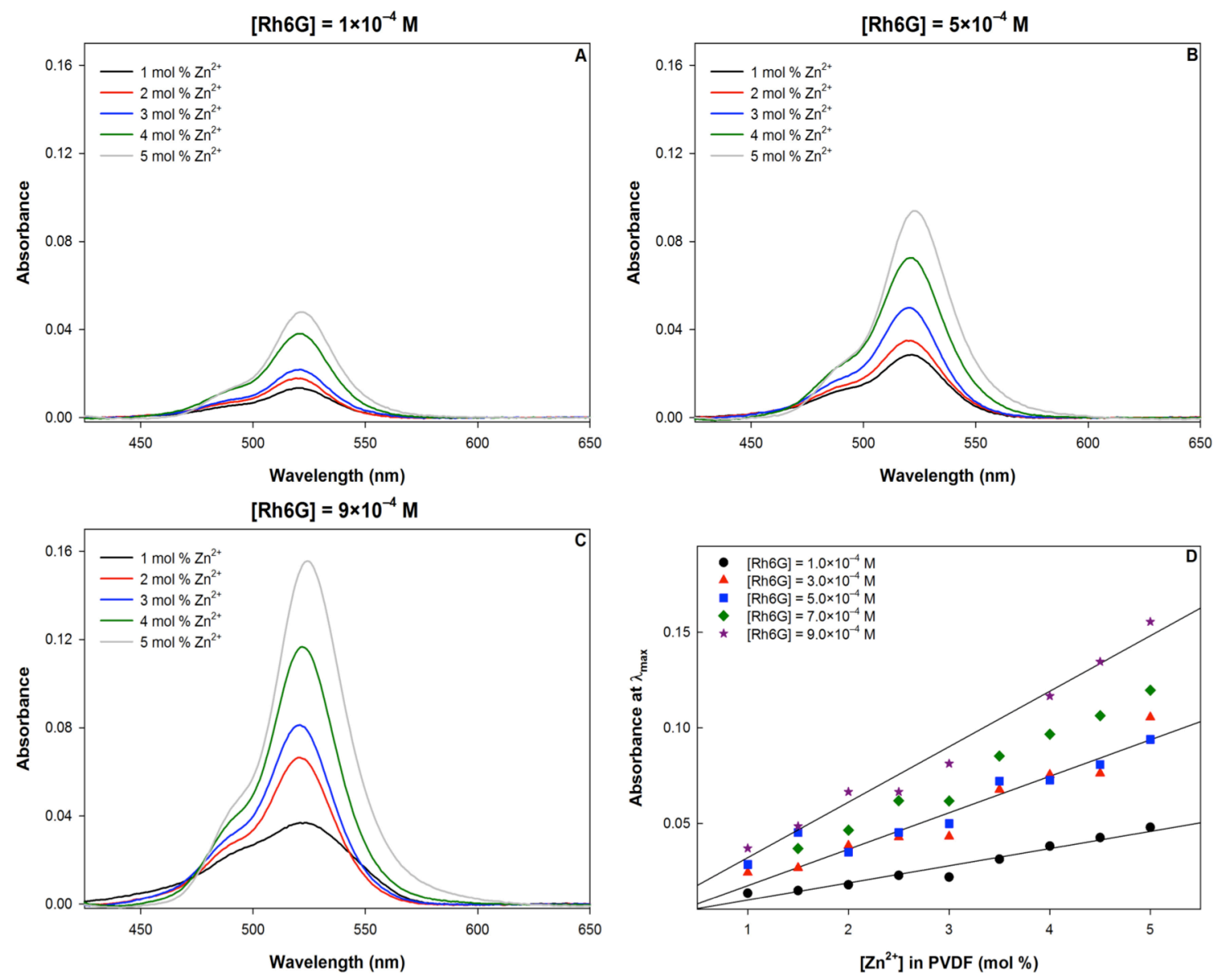

Figure 5. Absorbance spectra of Rh6G on PVDF surfaces with different $\mathrm{Zn}^{2+}$ doping levels. A: $1 \times 10^{-4}$ M Rh6G cast onto $\mathrm{Zn}^{2+}$ doped PVDF; B: $5 \times 10^{-4} \mathrm{M}$ Rh6G cast onto $\mathrm{Zn}^{2+}$ doped PVDF; C: $9 \times 10^{-4} \mathrm{M}$ Rh6G cast onto $\mathrm{Zn}^{2+}$ doped PVDF; D: absorbance at $1_{\max }$ on $\mathrm{Zn}^{2+}$ doped PVDF vs. for different Rh6G spin-casting concentration. The lines show the linear relationship for three of the concentrations, $1 \times 10^{-4} \mathrm{M}, 5 \times 10^{-4} \mathrm{M}$, and $9 \times 10^{-4} \mathrm{M}$ (others are not shown for the sake of clarity). Note that the absorbance scales are the same for A, B, and C.

The $\mathrm{Zn}^{2+}$ in the PVDF film also affects the spectral intensity, as shown in Figure 5. As the $\mathrm{Zn}^{2+}$ concentration increases in the film the intensity of the absorption increases even though the surface coverage of the Rh6G should be constant. This indicates that the zinc ion is interacting with surface Rh6G in such a fashion to affect the absorbance intensity but not the absorption energy. 
All of the absorbance line shapes are similar. All of the spectra were fit to three peaks and these results are given in Table 1. The peak maxima are similar to those reported previously. ${ }^{32,33}$ The peak at $521 \mathrm{~nm}$ is assigned to the monomer absorption while the features at $496 \mathrm{~nm}$ and $534 \mathrm{~nm}$ are assigned to an exciton pair with an oblique geometry. Even for the thickest films there is no evidence of a peak in the 560 $\mathrm{nm}$ region, which would be indicative of aggregation of the $\mathrm{Rh} 6 \mathrm{G}$ on the surface.

Table 1. Deconvoluted Gaussian peaks for the absorbance spectra. Peak position $\left(\lambda_{\max }\right)$ and FWHM $(\Gamma)$ have an uncertainty of $\pm 2 \mathrm{~nm}$.

\begin{tabular}{|c|c|c|}
\hline Peak & $\lambda_{\max }(\mathrm{nm})$ & $\Gamma(\mathrm{nm})$ \\
\hline 1 & 496 & 25 \\
\hline 2 & 521 & 15 \\
\hline 3 & 534 & 22 \\
\hline
\end{tabular}

Figures 6 and 7 shows the emission spectra for Rh6G coated on PVDF with various levels of $\mathrm{Zn}^{2+}$ doping. Unlike the absorption spectra, there are significant changes in the emission spectra as the coating conditions change. All of the emission spectra are broad. As the amount of Rh6G used in spin-casting or as the amount of $\mathrm{Zn}^{2+}$ doped into the PVDF increases, the peak maxima shift to lower energy. Concurrent with this shift in the maxima is the unveiling of a second peak at high energy, but lower intensity. The magnitudes of the changes are greater for higher $\mathrm{Zn}^{2+}$ concentrations or thicker Rh6G coatings. At the lowest concentration of Rh6G used in spin-casting $\left(1 \times 10^{-4} \mathrm{M}\right)$ the emission maximum increases from $562 \mathrm{~nm}$ at $1 \mathrm{~mol} \%$ $\mathrm{Zn}^{2+}$ to $578 \mathrm{~nm}$ for $5 \mathrm{~mol} \% \mathrm{Zn}^{2+}$, a shift of $16 \mathrm{~nm}$. In contrast, the corresponding emission maxima for $9 \times 10^{-4} \mathrm{M}$ are $575 \mathrm{~nm}$ to $618 \mathrm{~nm}$, an increase of $43 \mathrm{~nm}$. Likewise, at constant $\mathrm{Zn}^{2+}$ doping the changes are $13 \mathrm{~nm}$ at $1 \mathrm{~mol} \%$ and $40 \mathrm{~nm}$ for 5 
mol\%. The changes in the emission spectra are similar for either more $\mathrm{Zn}^{2+}$ or more Rh6G and the two structural changes amplify the emission changes.

Similar behavior is observed for the total emission intensity, as shown in Figure 8. As more Rh6G is added to the surface the total emission intensity increases, which makes sense since there is more fluorophore present on the surface. However, as the amount of $\mathrm{Zn}^{2+}$ doped into the PVDF increases, the total emission intensity also increases, even at a constant amount of Rh6G.
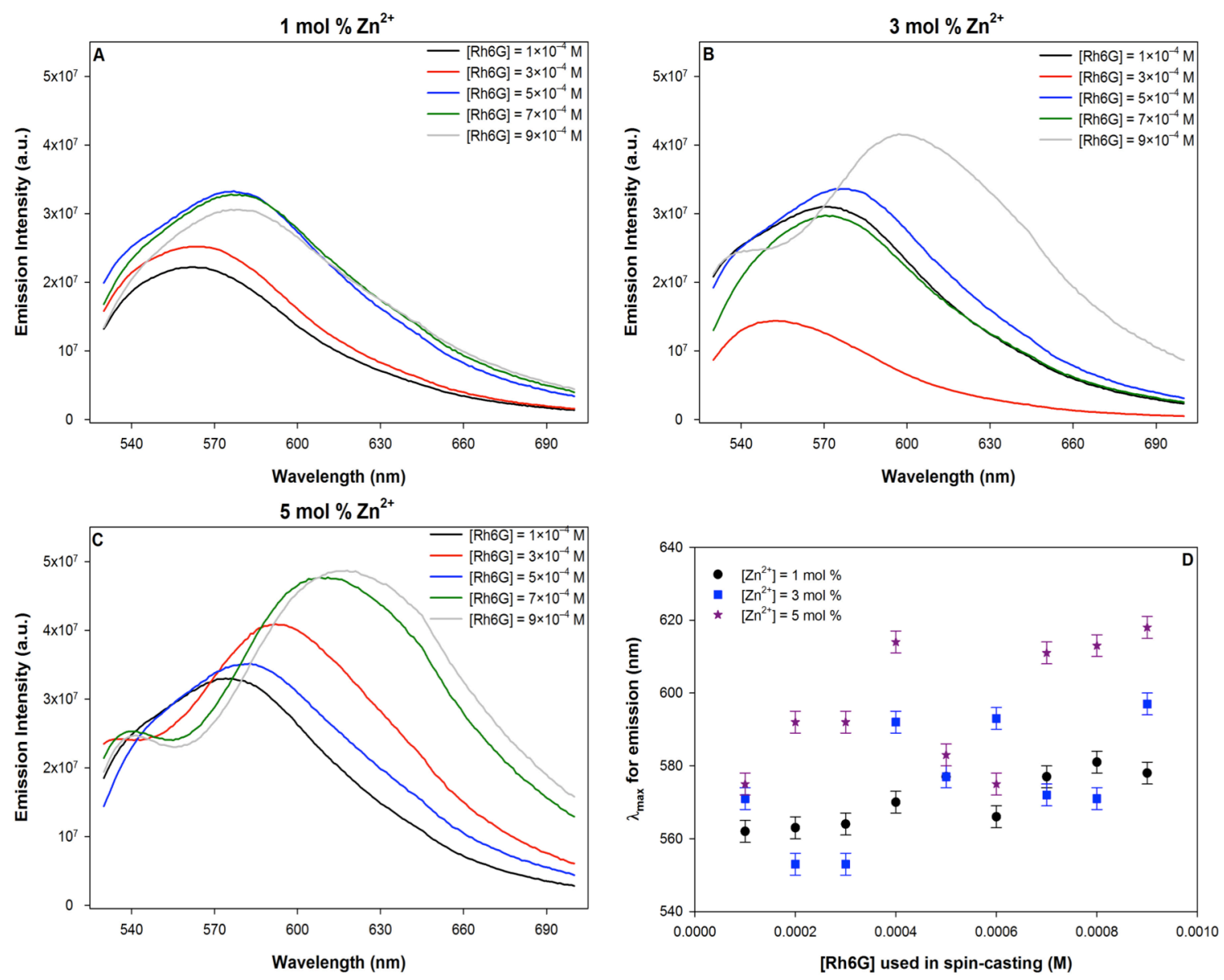

Figure 6. Emission spectra as a function of Rh6G concentration used in spin-casting. A: cast onto PVDF/1 mol \% $\mathrm{Zn}^{2+}$; $\mathrm{B}$ : cast onto $\mathrm{PVDF} / 3 \mathrm{~mol} \% \mathrm{Zn}^{2+}$; C: cast onto $\mathrm{PVDF} / 5 \mathrm{~mol} \% \mathrm{Zn}^{2+}$; D: $1_{\max }$ vs. spin-casting concentration for different $\mathrm{Zn}^{2+}$ doping levels. Note that the intensity scales are the same for A, B, and C. 

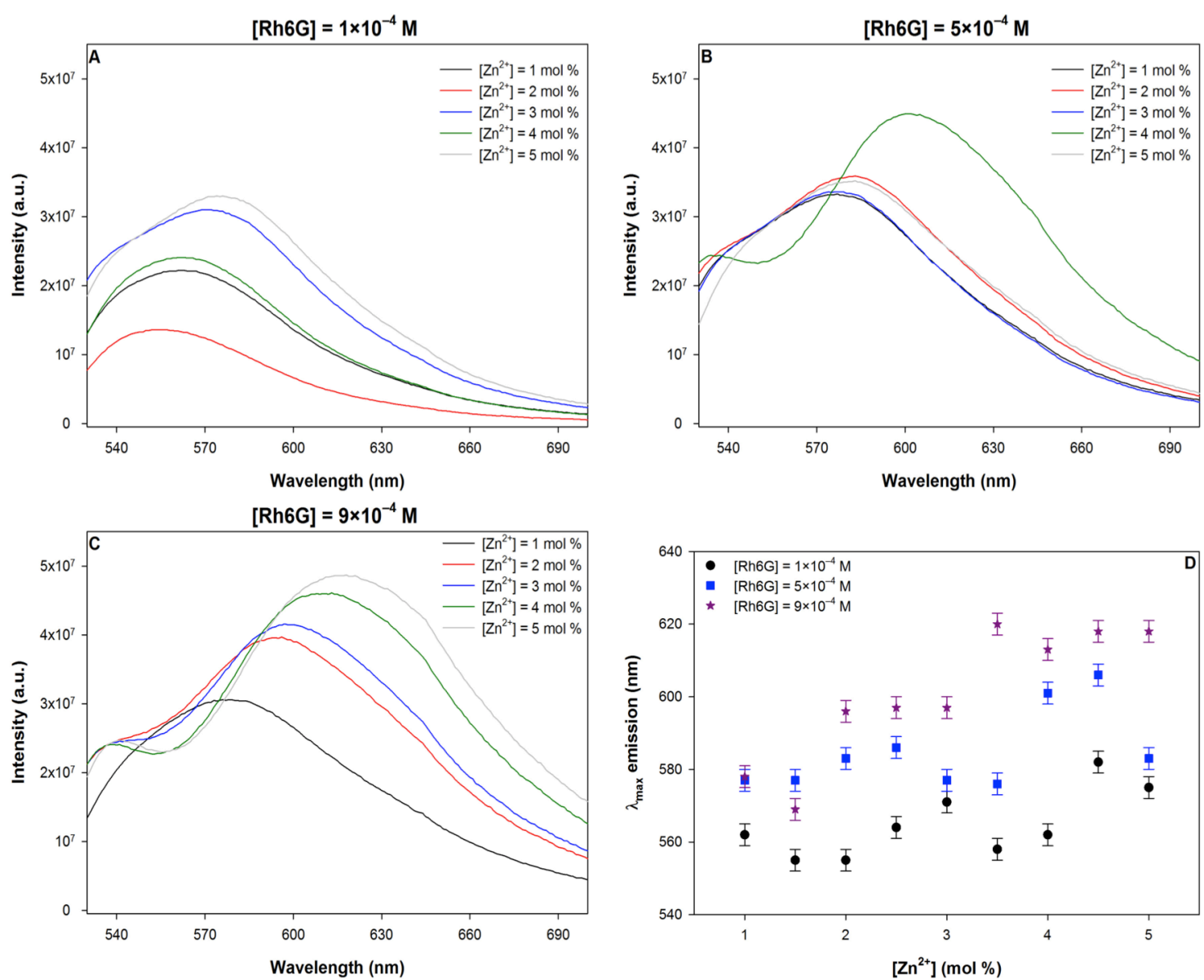

Figure 7. Emission spectra of Rh6G on PVDF surfaces with different $\mathrm{Zn}^{2+}$ doping levels. A: $1 \times 10^{-4} \mathrm{M}$ Rh6G cast onto $\mathrm{Zn}^{2+}$ doped PVDF; B: $5 \times 10^{-4} \mathrm{M}$ Rh6G cast onto $\mathrm{Zn}^{2+}$ doped PVDF; C: $9 \times 10^{-4} \mathrm{M}$ Rh6G cast onto $\mathrm{Zn}^{2+}$ doped PVDF; D: $1_{\max }$ on $\mathrm{Zn}^{2+}$ doped PVDF vs. for different Rh6G spin-casting concentration. Note that the intensity scales are the same for $\mathrm{A}, \mathrm{B}$, and $\mathrm{C}$.
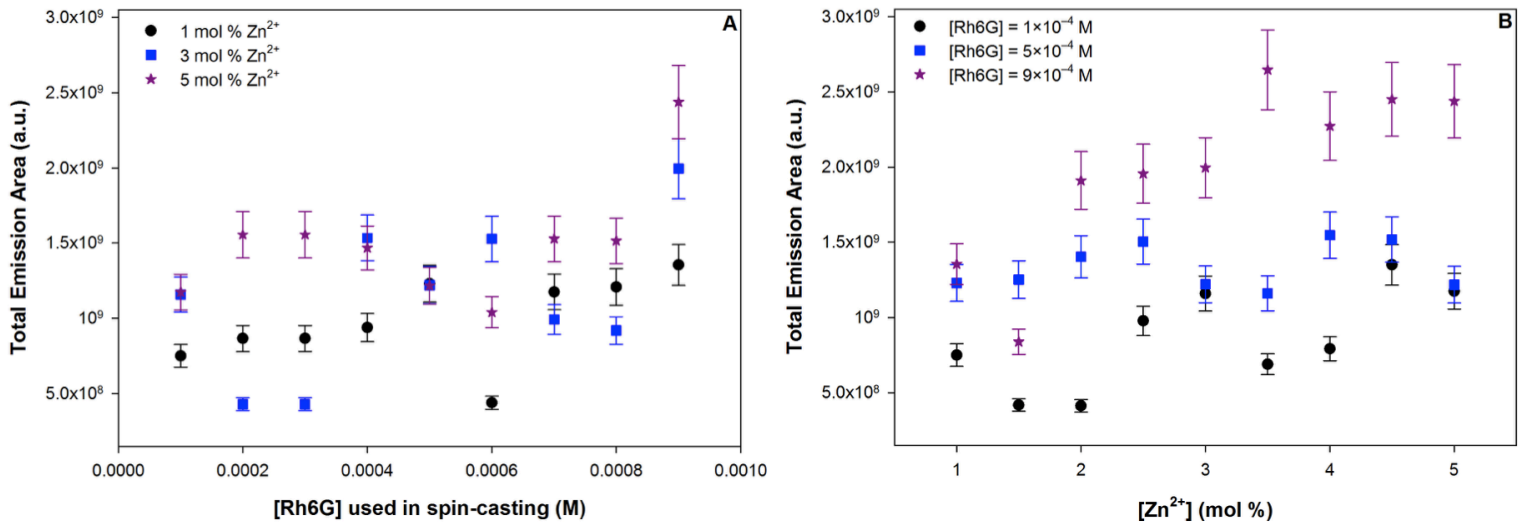

Figure 8. Total area under the emission spectra as a function of: $A$ [Rh6G] used in spin-casting and $\mathrm{B} \mathrm{Zn}^{2+}$ doping level in the PVDF film. 
Each of the emission spectra can be deconvoluted into three peaks. As shown in Figure 9, the highest energy peak is found at $536 \mathrm{~nm}$ and is fairly constant $( \pm 4 \mathrm{~nm})$ from low to high $\mathrm{Zn}^{2+}$ levels or from low to high Rh6G levels. At low Rh6G coverage, the other two peaks $(\sim 560 \mathrm{~nm}$ and $\sim 600 \mathrm{~nm})$ are only slightly affected by the concentration of the $\mathrm{Zn}^{2+}$ in the underlying polymer layer. However, as the coverage of the Rh6G increases, the influence of the $\mathrm{Zn}^{2+}$ also increases so that at the highest Rh6G thickness the peaks shift from $\sim 570 \mathrm{~nm}$ to $\sim 615 \mathrm{~nm}\left(\omega_{2}\right)$ and $\sim 605 \mathrm{~nm}$ to $\sim 675$ $\mathrm{nm}\left(\omega_{3}\right)$.

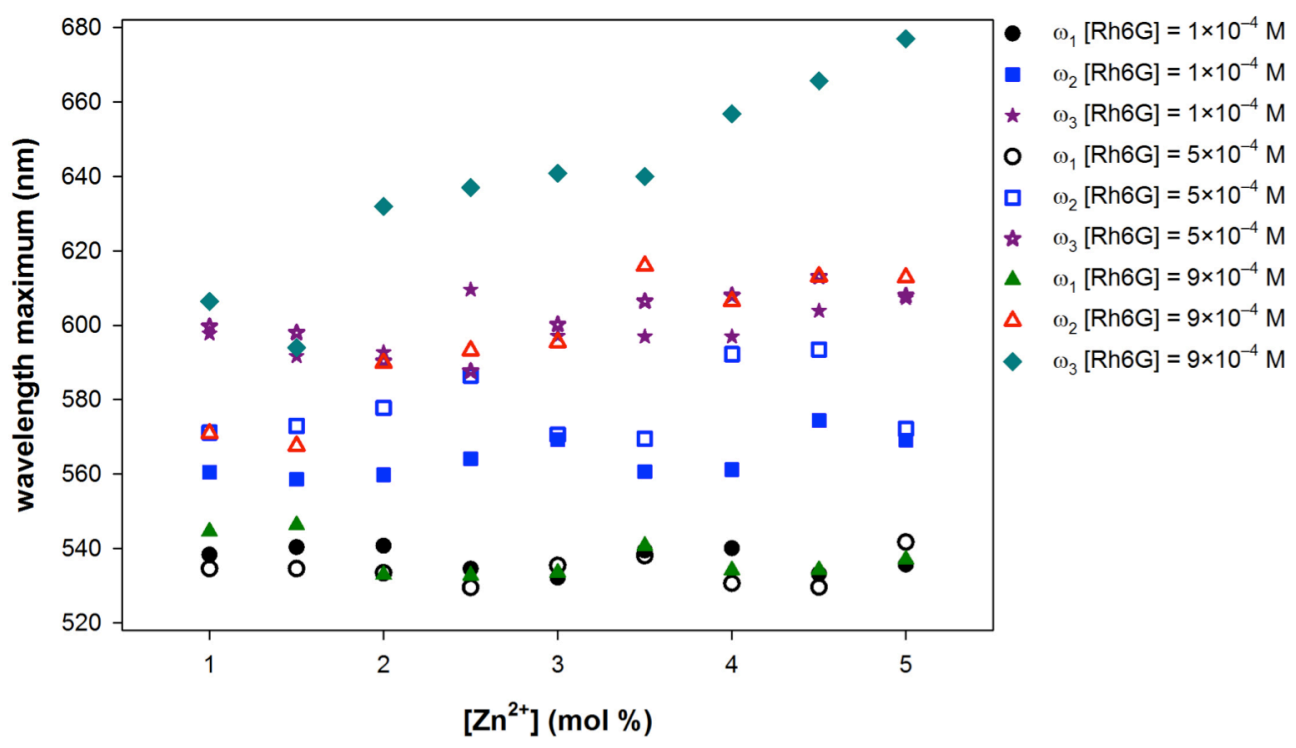

Figure 9. Wavelength maxima found from deconvolution of the emission spectra. Errors in each data point are $\pm 3 \mathrm{~nm}$.

Figure 10 shows the normalized excitation, absorbance, and emission spectra for selected samples of Rh6G spin-cast onto $\mathrm{Zn}^{2+}$-doped PVDF. In all cases the excitation spectra and the absorption spectra match reasonably well. Even in the cases where the emission maximum is at the lowest energy, the excitation spectra do not show peaks in the low energy region. This requires that the low energy emission 
observed in the $625-650 \mathrm{~nm}$ region must arise from excitation from the higher energy monomer or exciton states.
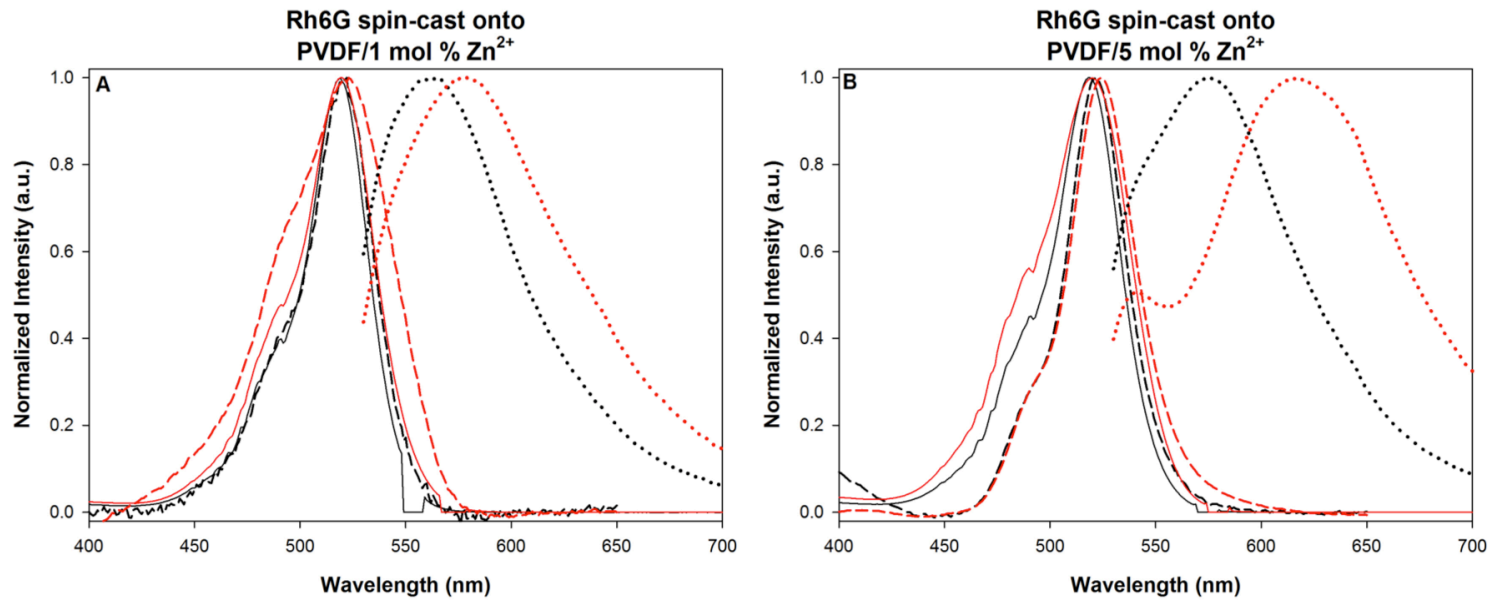

Figure 10. Normalized excitation (solid lines, detection done at $1_{\max }$ of the emission spectrum), absorbance (dashed lines), and emission (dotted lines) spectra for Rh6G on $\mathrm{Zn}^{2+}$-doped PVDF. A: $1 \mathrm{~mol} \% \mathrm{Zn}^{2+}$ in PVDF. B: $5 \mathrm{~mol} \% \mathrm{Zn}^{2+}$ in PVDF. For both figures: black lines $-[\mathrm{Rh} 6 \mathrm{G}]$ used in spin-casting $=1 \times 10^{-4} \mathrm{M}$; red lines $-[\mathrm{Rh} 6 \mathrm{G}]$ used in spin-casting $=9 \times 10^{-4} \mathrm{M}$.

Figure 11 shows the decay curves for different samples. Other than baseline differences, all of the decay curves overlap. This demonstrates that the lifetimes of Rh6G are not significantly affected by the film thickness or the nature of the underlying substrate. The decay curves are best fit using three exponential functions giving three lifetime parameters. The shortest lifetime was always less than $0.5 \mathrm{~ns}$ and is assigned to scattering from the film. The other two lifetimes are associated with the $\mathrm{Rh} 6 \mathrm{G}$ and are given in Table 2. The shorter lifetime is $\tau_{1}=2.4 \pm 0.4 \mathrm{~ns}$ is assigned to relaxation from the Rh6G isolated monomer. In dilute DMF solution the lifetime of Rh6G is $4.2 \mathrm{~ns},{ }^{10}$ which indicates that in the thin film the underlying substrate provides more nonradiative pathways than in solvent. The longer lifetime, $\tau_{2}=$ $10.4 \pm 1.6 \mathrm{~ns}$, is assigned to de-excitation from aggregated areas of the thin film. Since 
there is no direct absorption measured for aggregates, the longer lived excited states must arise from energy transfer or charge transfer from the monomer or exciton. Another source of this longer lifetime could be exciton diffusion within the Rh6G thin film.

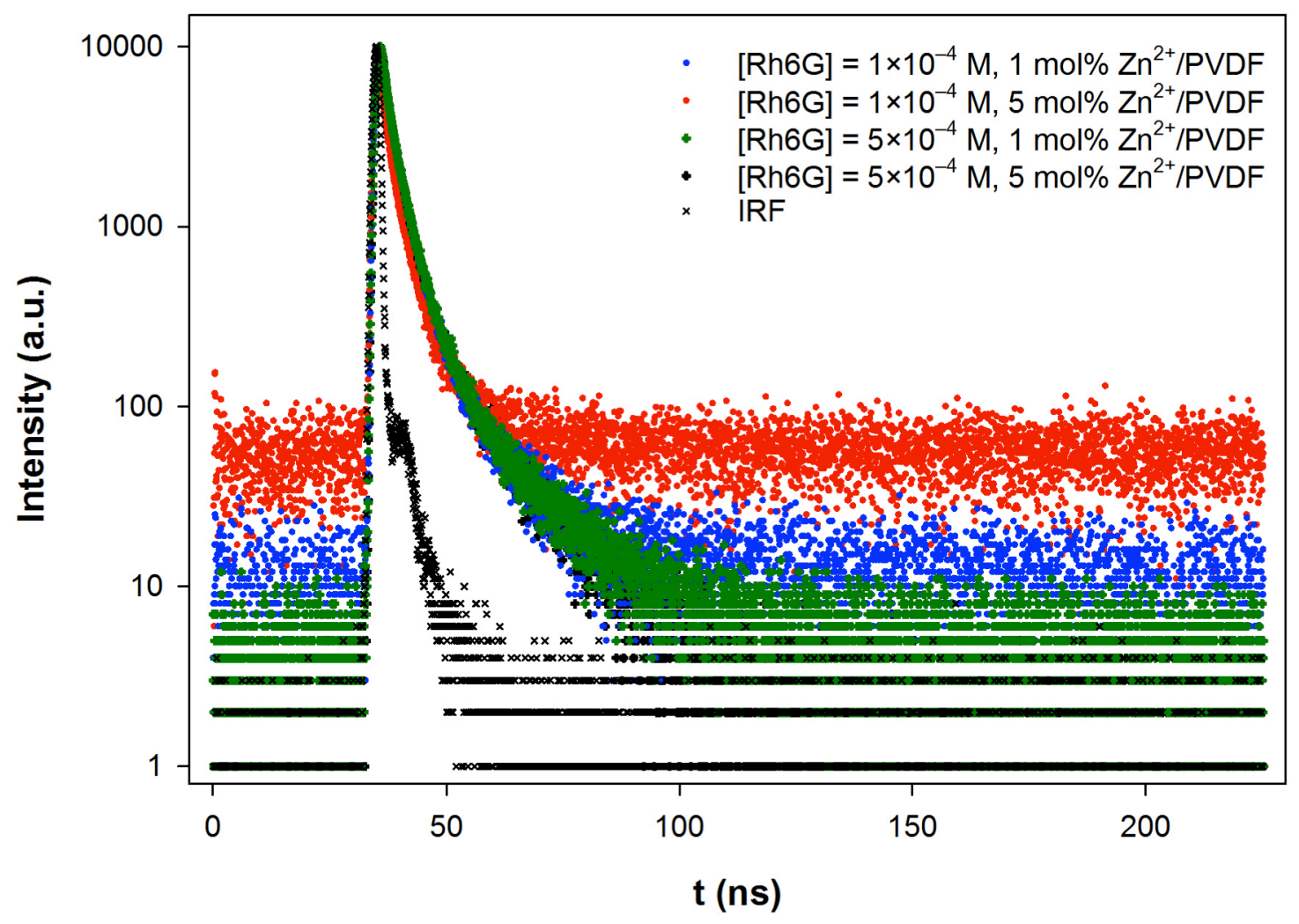

Figure 11. Excited state decays for $\mathrm{Rh} 6 \mathrm{G}$ on $\mathrm{Zn}^{2+}$-doped $\mathrm{PVDF}$. IRF $=$ instrument response function. 
Table 2. Measured excited state life times for Rh6G thin films on $\mathrm{Zn}^{2+}$-doped PVDF. $\mathrm{c}^{2}<1.2$ for all fits.

\begin{tabular}{|c|c|c|}
\hline & $\tau_{1}(\mathrm{~ns})$ & $\tau_{2}(\mathrm{~ns})$ \\
\hline $1 \mathrm{~mol} \% \mathrm{Zn}^{2+}, 1 \times 10^{-4} \mathrm{M}$ Rh6G & 2.6 & 11.7 \\
\hline $1 \mathrm{~mol} \% \mathrm{Zn}^{2+}, 5 \times 10^{-4} \mathrm{M}$ Rh6G & 2.5 & 12.7 \\
\hline $1 \mathrm{~mol} \% \mathrm{Zn}^{2+}, 9 \times 10^{-4} \mathrm{M}$ Rh6G & 2.4 & 10.0 \\
\hline $3 \mathrm{~mol} \% \mathrm{Zn}^{2+}, 1 \times 10^{-4} \mathrm{M}$ Rh6G & 3.1 & 11.8 \\
\hline $3 \mathrm{~mol} \% \mathrm{Zn}^{2+}, 5 \times 10^{-4} \mathrm{M}$ Rh6G & 2.1 & 8.3 \\
\hline $3 \mathrm{~mol} \% \mathrm{Zn}^{2+}, 9 \times 10^{-4} \mathrm{M}$ Rh6G & 1.9 & 8.4 \\
\hline $5 \mathrm{~mol} \% \mathrm{Zn}^{2+}, 1 \times 10^{-4} \mathrm{M}$ Rh6G & 2.1 & 8.2 \\
\hline $5 \mathrm{~mol} \% \mathrm{Zn}^{2+}, 5 \times 10^{-4} \mathrm{M}$ Rh6G & 2.2 & 11.8 \\
\hline $5 \mathrm{~mol} \% \mathrm{Zn}^{2+}, 9 \times 10^{-4} \mathrm{M}$ Rh6G & 2.8 & 10.8 \\
\hline Average & 2.4 & 10.4 \\
\hline Standard deviation & 0.4 & 1.6 \\
\hline
\end{tabular}

As summarized in Table 3, the presence of the $\mathrm{Zn}^{2+}$ ion in the PVDF film has a profound effect on the photophysics of Rh6G. In the absorption spectra, the intensity of absorption increases for both increased Rh6G thickness and increased $\mathrm{Zn}^{2+}$. However, absorption associated with aggregation is completely suppressed. An electric field created by the $\mathrm{Zn}^{2+}$ could increase the transition moment of the monomer but why this would eliminate aggregate absorption is not known. In the emission spectra the $\mathrm{Zn}^{2+}$ again has important effects. As $\mathrm{Rh} 6 \mathrm{G}$ or $\mathrm{Zn}^{2+}$ increases, the spectral intensity increases and the maxima shift to lower energy. 
Table 3. Summary of effects on $\mathrm{Rh} 6 \mathrm{G}$ by $\mathrm{Zn}^{2+}$-doped PVDF. The data for no $\mathrm{Zn}^{2+}$ are taken from reference 33 .

\begin{tabular}{|c|c|c|}
\hline & No $\mathbf{Z n}^{2+}$ & With $\mathbf{Z n}^{2+}$ \\
\hline \multicolumn{3}{|l|}{ Absorption spectra } \\
\hline Rh6G thickness increases & $\begin{array}{c}\text { Increases nonlinearly } \\
\text { New peak grows in at low } \\
\text { energy }\end{array}$ & $\begin{array}{l}\text { Increases linearly } \\
\text { No new peaks }\end{array}$ \\
\hline $\begin{array}{c}\mathrm{Zn}^{2+} \text { concentration } \\
\text { increases }\end{array}$ & & $\begin{array}{l}\text { Increases linearly } \\
\text { No new peaks }\end{array}$ \\
\hline \multicolumn{3}{|l|}{ Excitation spectra } \\
\hline Rh6G thickness increases & $\begin{array}{c}\text { Matches high energy absorption } \\
\text { peaks }\end{array}$ & $\begin{array}{c}\text { Matches entire } \\
\text { absorption spectrum }\end{array}$ \\
\hline $\begin{array}{l}\mathrm{Zn}^{2+} \text { concentration } \\
\text { increases }\end{array}$ & & $\begin{array}{c}\text { Matches entire } \\
\text { absorption spectrum }\end{array}$ \\
\hline \multicolumn{3}{|l|}{ Emission spectra } \\
\hline Rh6G thickness increases & $\begin{array}{l}\omega_{1}, \omega_{2}, \text { and } \omega_{3} \text { stay constant } \\
\omega_{4} \text { grows in at lower energy }\end{array}$ & $\begin{array}{c}\omega_{1} \text { stays constant } \\
\omega_{2} \text { and } \omega_{3} \text { shift to } \\
\text { lower energy }\end{array}$ \\
\hline $\begin{array}{l}\mathrm{Zn}^{2+} \text { concentration } \\
\text { increases }\end{array}$ & & $\begin{array}{c}\omega_{1} \text { stays constant } \\
\omega_{2} \text { and } \omega_{3} \text { shift to } \\
\text { lower energy }\end{array}$ \\
\hline \multicolumn{3}{|l|}{ Excited state lifetimes } \\
\hline Rh6G thickness increases & $\tau_{1}$ and $\tau_{2}$ decrease & $\begin{array}{c}\tau_{1} \text { and } \tau_{2} \text { remain } \\
\text { constant }\end{array}$ \\
\hline $\begin{array}{l}\mathrm{Zn}^{2+} \text { concentration } \\
\text { increases }\end{array}$ & & $\begin{array}{c}\tau_{1} \text { and } \tau_{2} \text { remain } \\
\text { constant }\end{array}$ \\
\hline
\end{tabular}

A tentative explanation is as follows. At low Rh6G surface coverages, the Rh6G molecules are mostly well separated from each other but with some Rh6G molecules having close nearest neighbors. Absorption of light is mainly from monomers but with some exciton states available. The orientation of the nearest neighbor Rh6G molecules is in an oblique geometry so that absorption to both of the high and low energy exciton states is allowed. The ionic component and ferroelectric structural components in the underlying thin film create an electric field in the Rh6G layer that increases the transition moment of the $\mathrm{Rh} 6 \mathrm{G}$ as the amount of $\mathrm{Zn}^{2+}$ ion increases. Likewise, at low Rh6G, the underlying substrate only affects the emission 
intensity. At high Rh6G coverage, the absorption increases because of the increased material but no absorption associated with aggregation is observed. However, in the emission spectra thick Rh6G films are more complicated. As the $\mathrm{Zn}^{2+}$ ion increases, there is a significant shift of the emission maxima to lower energy, revealing a high energy emission not previously observed. Three of the emission features can be assigned to the monomer excited state and the two exciton excited states. The lowest energy emission must be either from aggregates populated by energy transfer or excitons that diffuse into the Rh6G thin film. Since the overlap of the absorption spectra and the emission spectra is quite small (see Fig. 10), exciton diffusion seems more likely, which is also consistent with the measured lifetimes.

\section{CONCLUSION}

Inclusion of $\mathrm{Zn}^{2+}$ ions into a PVDF film has significant effects both on the polymer film and at the interface between the PVDF and Rh6G. The zinc ion coordinates some of the DMF solvent, apparently generating a tetragonally distorted octahedral $\left[\mathrm{Zn}(\mathrm{DMF})_{6}\right]^{2+}$ complex. One outcome of this is that the PVDF layer has a larger component of the ferroelectric $\beta$-phase. The presence of the zinc complex, even at low doping levels, also has a profound impact on the photophysics of Rh6G on the surface. Most notably, aggregation of the Rh6G is suppressed, as evidenced by the absence of a low energy absorption peak for any Rh6G coverage level. Emission occurs from three Rh6G states, one of which is the first excited state of an isolated Rh6G molecule. At coverages of one or two monolayers there is also emission from exciton states. For the thickest Rh6G films, the emission intensity is highest, again implying the absence of aggregates. The presence of the $\mathrm{Zn}^{2+}$ complex in the PVDF 
appears to be promoting exciton diffusion, which modestly lengthens the lifetime of the excited state and lowers the emission energy but without significant quenching. 


\section{NOTES}

The authors declare no competing financial interest.

\section{ACKNOWLEDGMENT}

This material is based upon work supported by the U.S. Department of Homeland Security, Science and Technology Directorate, Office of University Programs, under Grant Award 2013-ST-061-ED0001. The views and conclusions contained in this document are those of the authors and should not be interpreted as necessarily representing the official policies, either expressed or implied, of the U.S. Department of Homeland Security. We also thank Matthew Conrad for assisting in the TGA experiments. 


\section{Supplementary Material}

\section{S1: Graphic.}

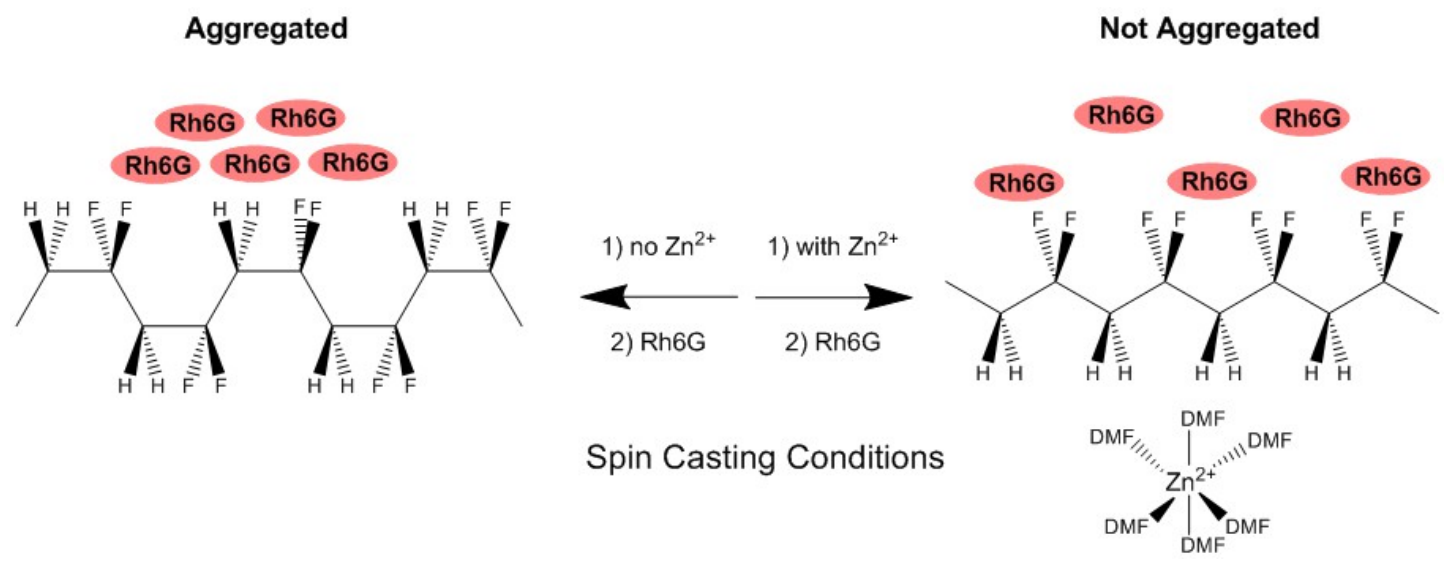


S2: Absorbance Spectrum of Rhodamine 6G on Glass Substrate.

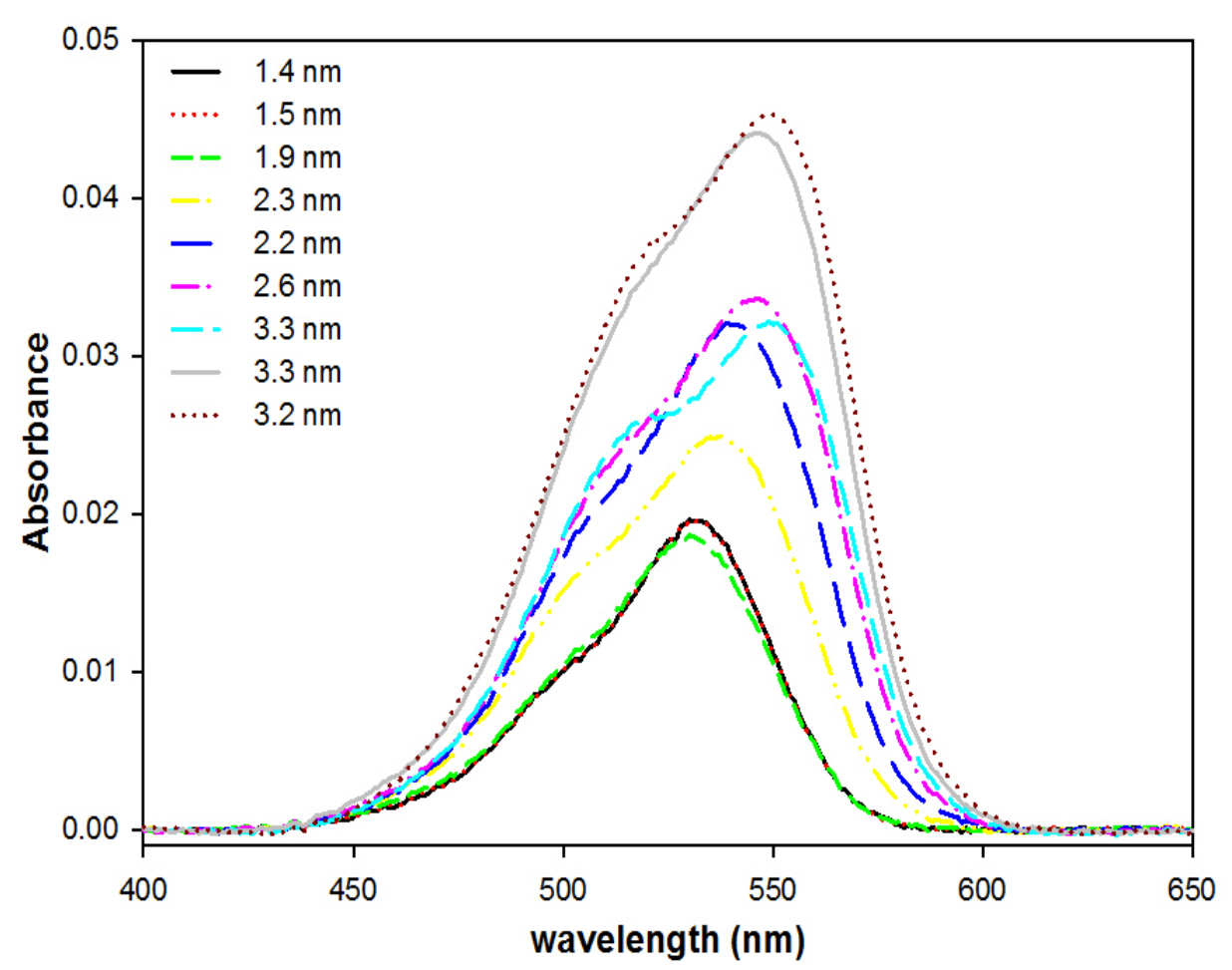

S3: Heat Effect of Different Concentration of Rhodamine 6G on Glass Substrate.

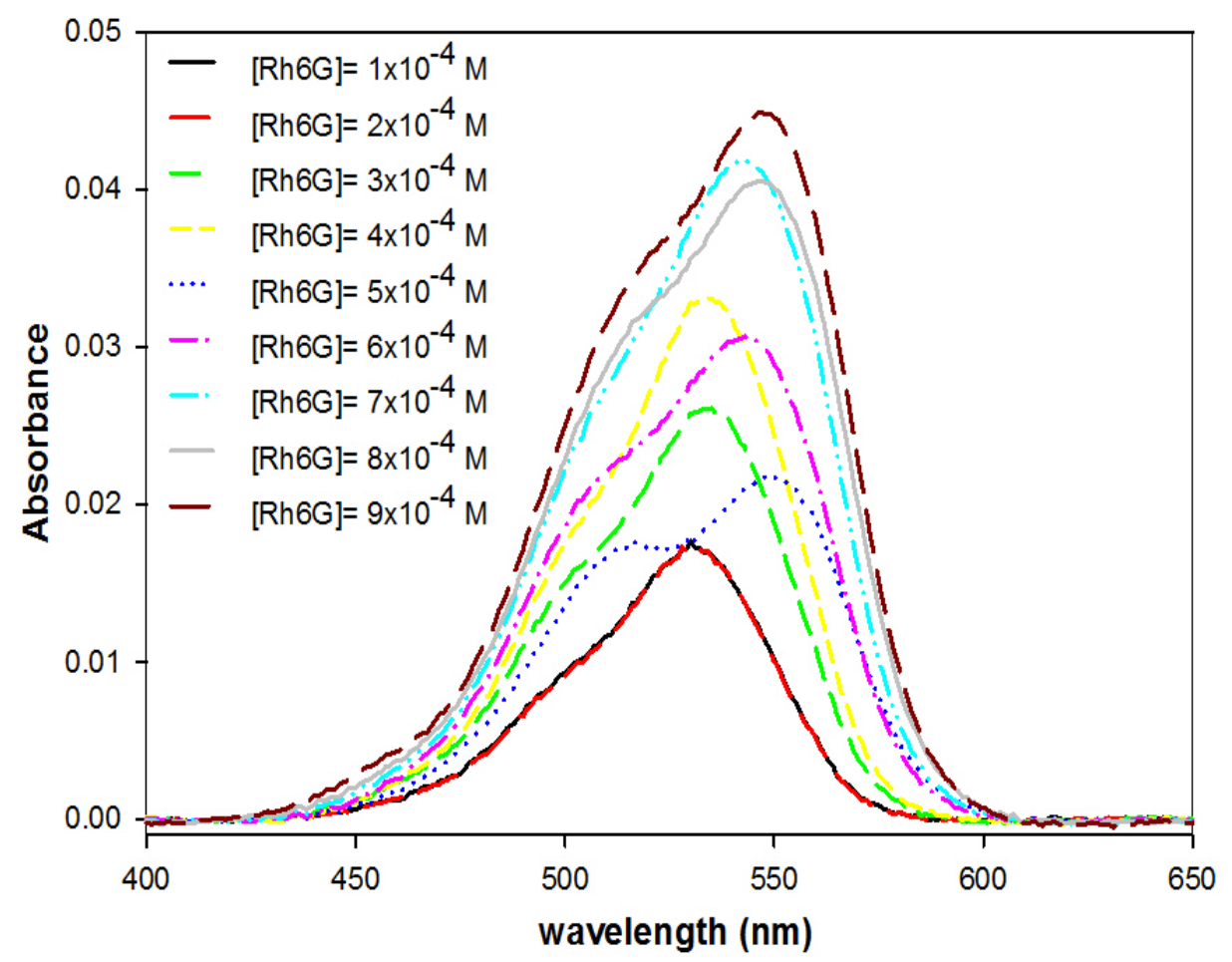


S4: Absorbance Spectrum of $1 \times 10^{-4}$ M Rh6G Deposition Solution Doped PVDF $/ \mathrm{Zn}^{2+}$ Substrate.

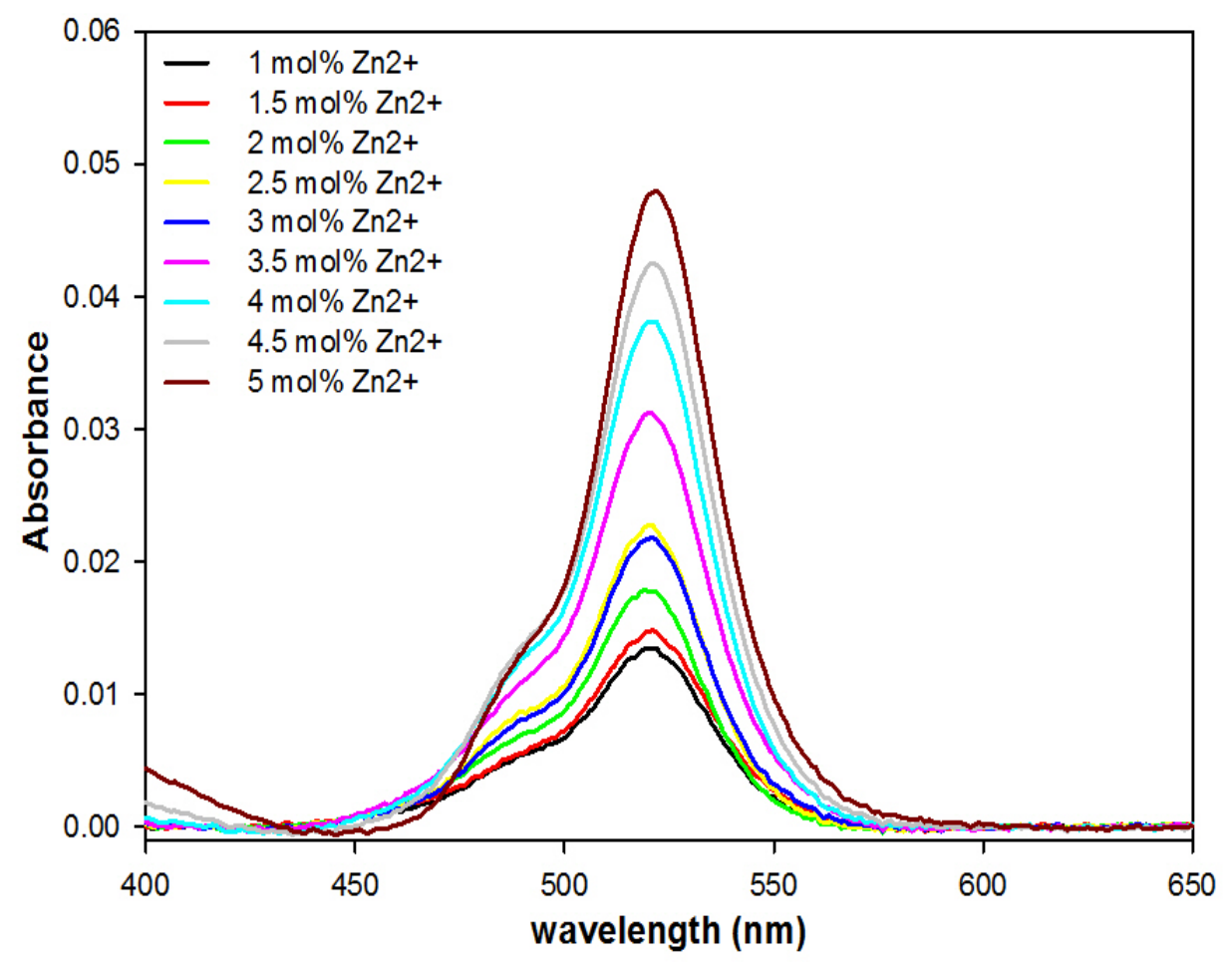

S5: Absorbance Spectrum of $2 \times 10^{-4}$ M Rh6G Deposition Solution Doped PVDF $/ \mathrm{Zn}^{2+}$ Substrate.

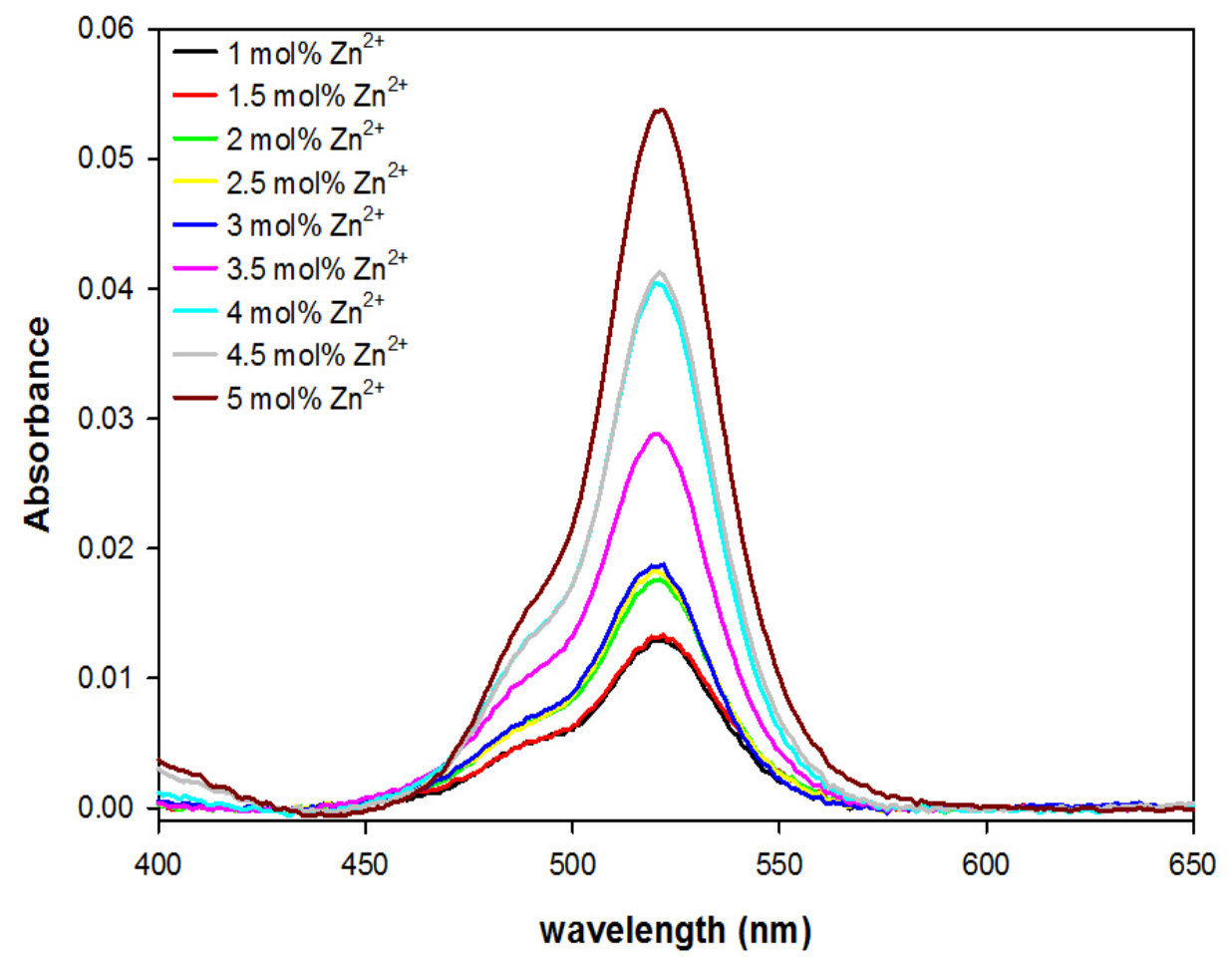


S6: Absorbance Spectrum of $3 \times 10^{-4}$ M Rh6G Deposition Solution Doped PVDF $/ \mathbf{Z n}^{2+}$ Substrate.

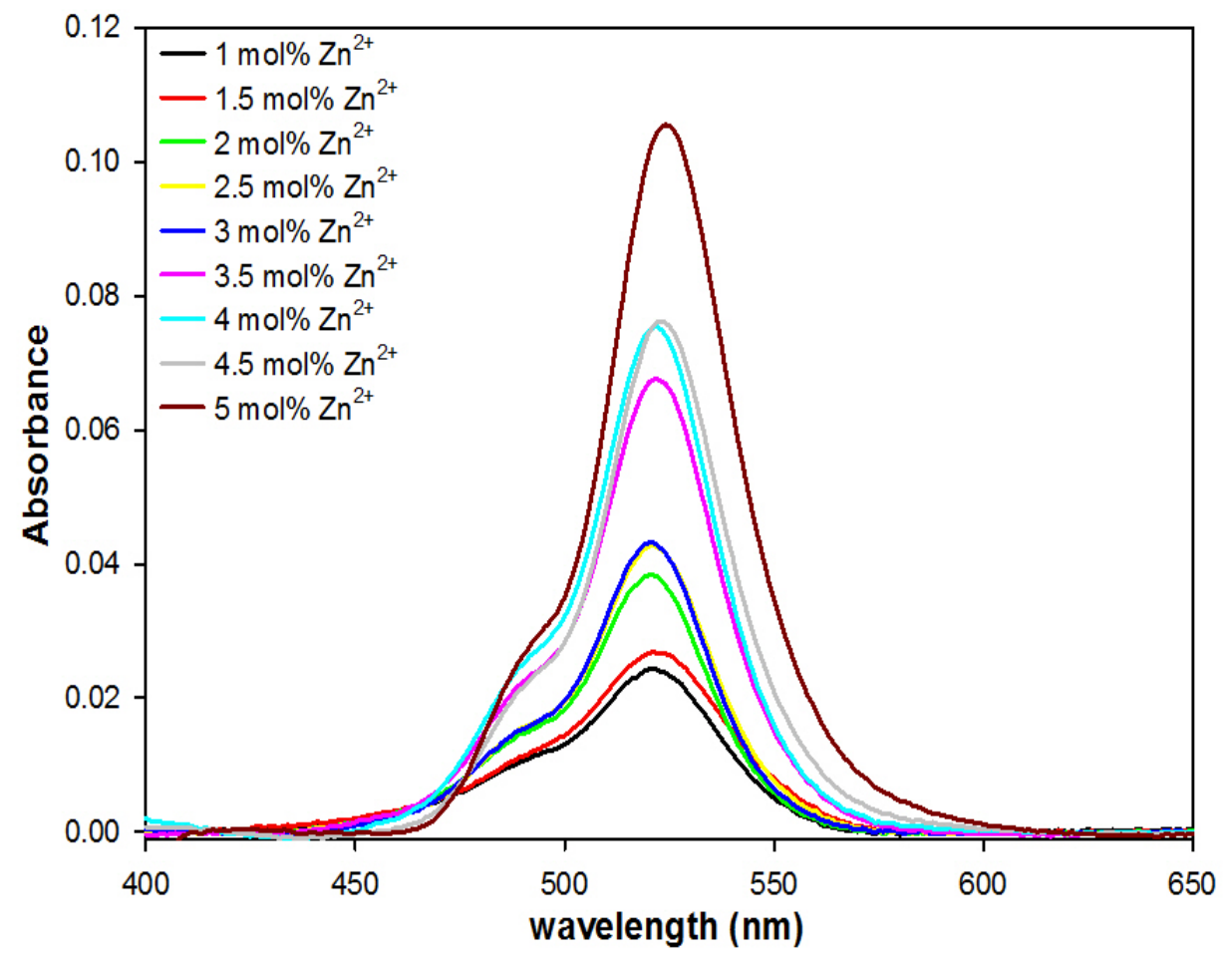

S7: Absorbance Spectrum of $4 \times 10^{-4}$ M Rh6G Deposition Solution Doped PVDF $/ Z^{2+}$ Substrate.

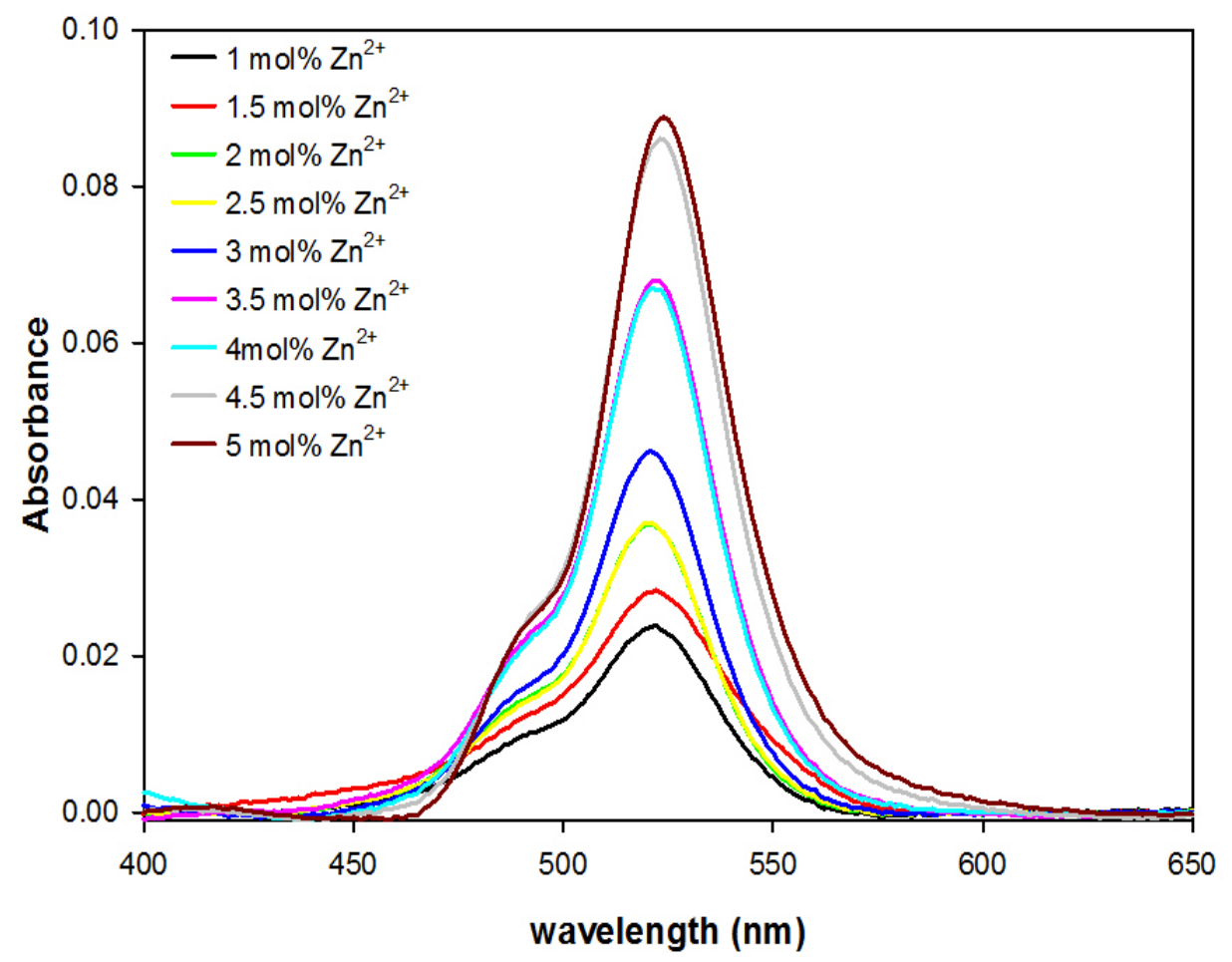


S8: Absorbance Spectrum of $5 \times 10^{-4}$ M Rh6G Deposition Solution Doped PVDF $/ \mathbf{Z n}^{2+}$ Substrate.

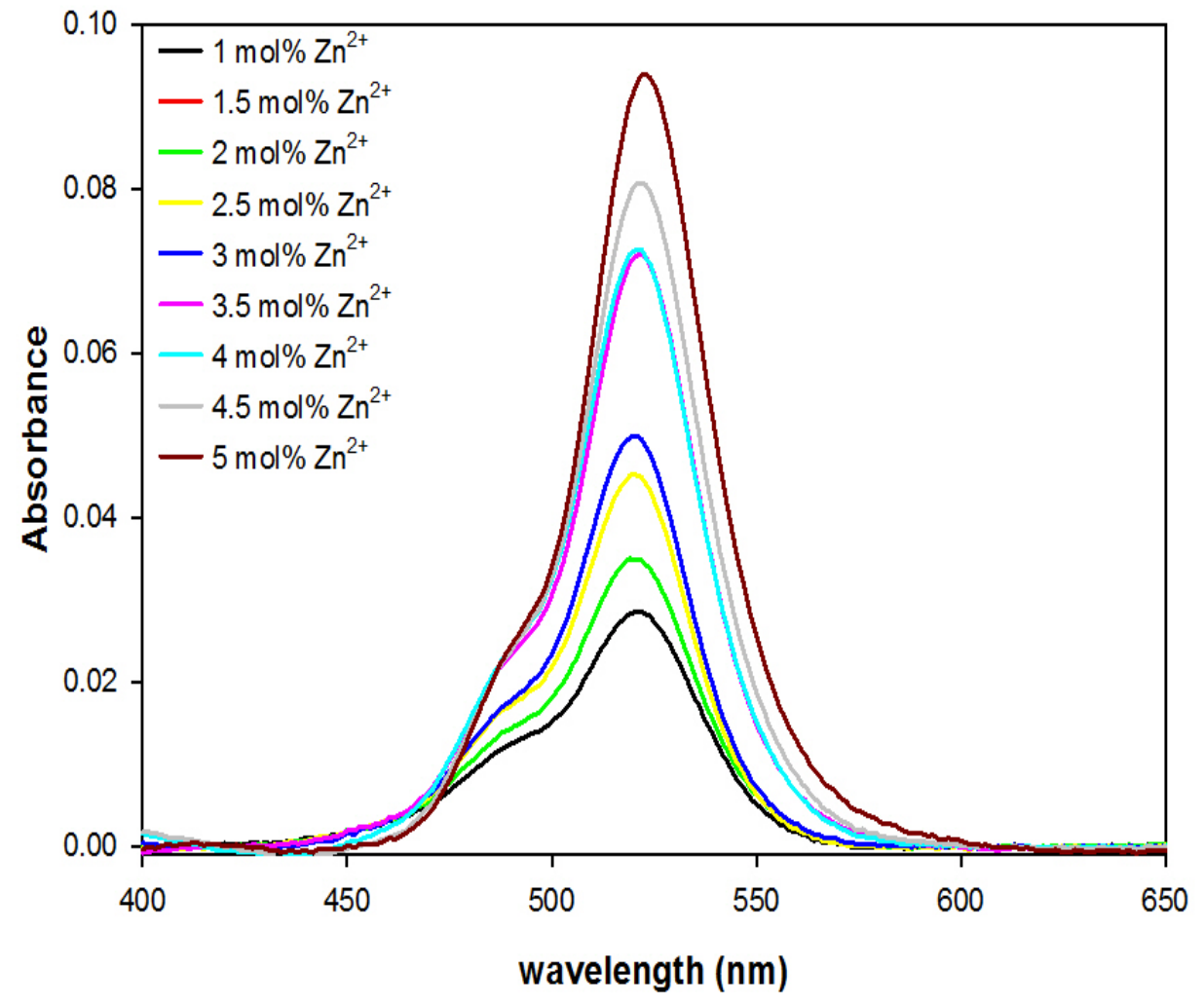

S9: Absorbance Spectrum of $6 \times 10^{-4}$ M Rh6G Deposition Solution Doped PVDF $/ \mathbf{Z n}^{2+}$ Substrate.

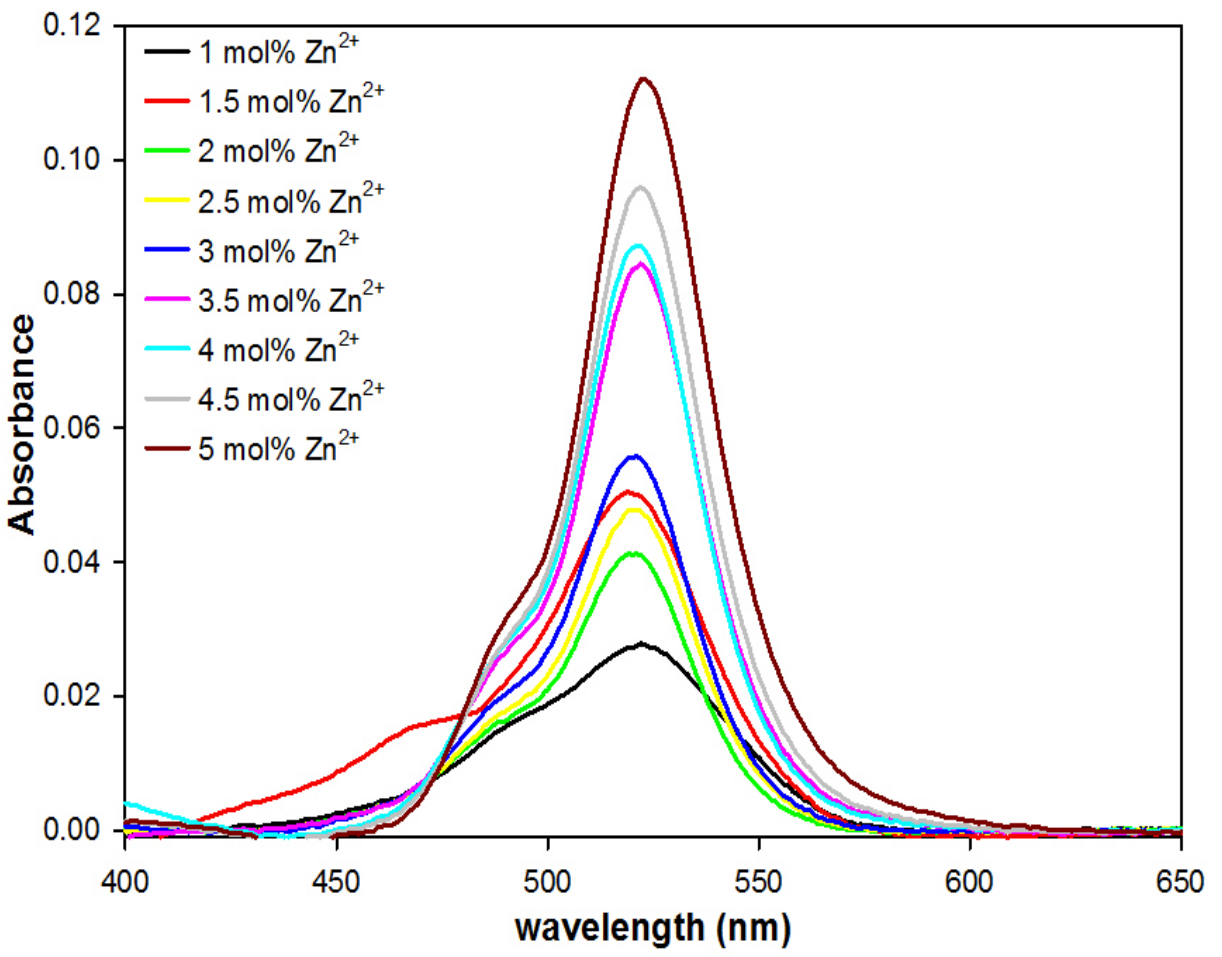


S10: Absorbance Spectrum of $7 \times 10^{-4}$ M Rh6G Deposition Solution Doped PVDF $/ \mathbf{Z n}^{2+}$ Substrate.

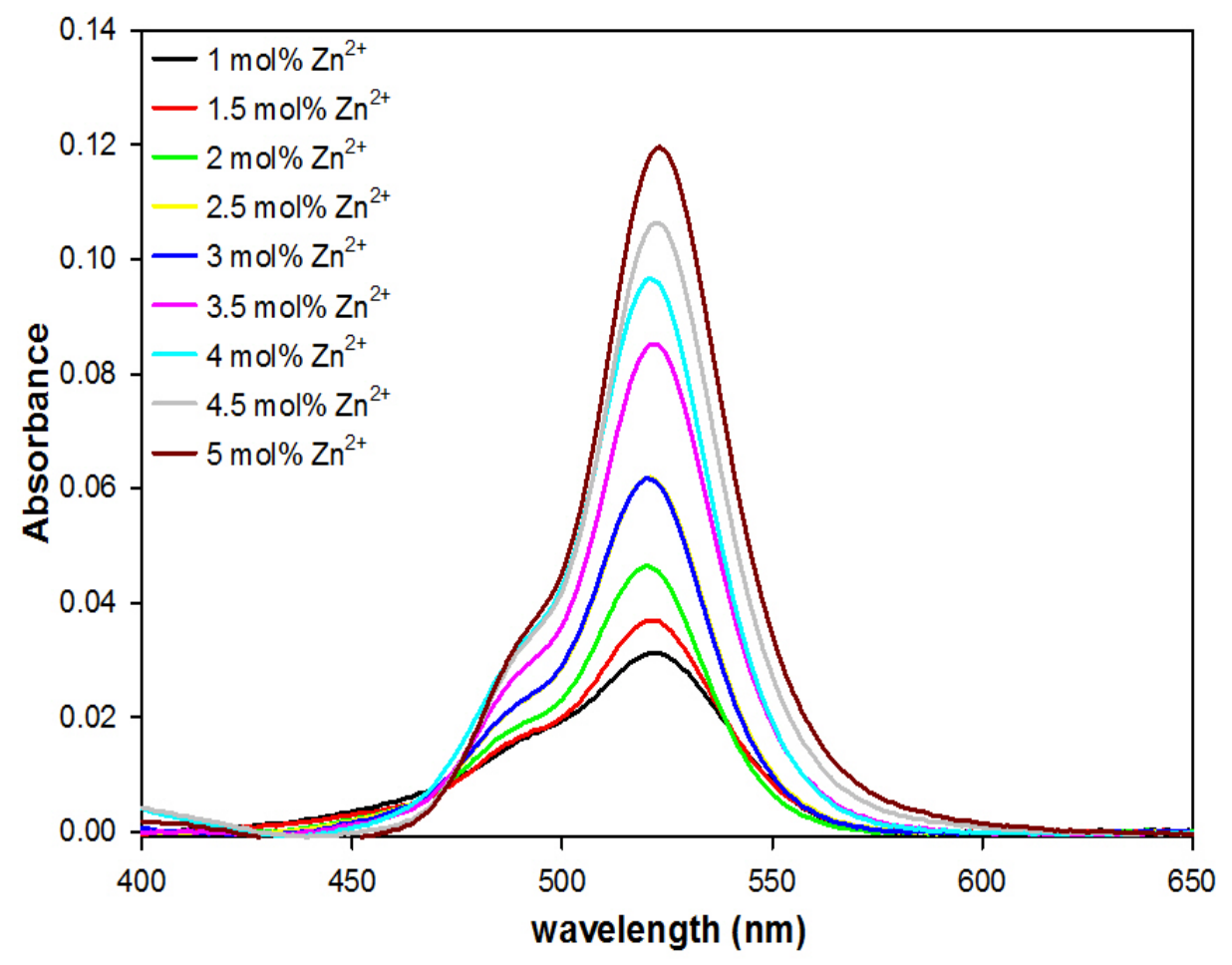

S11: Absorbance Spectrum of 8x10 ${ }^{-4}$ M Rh6G Deposition Solution Doped PVDF $/ Z^{2+}$ Substrate.

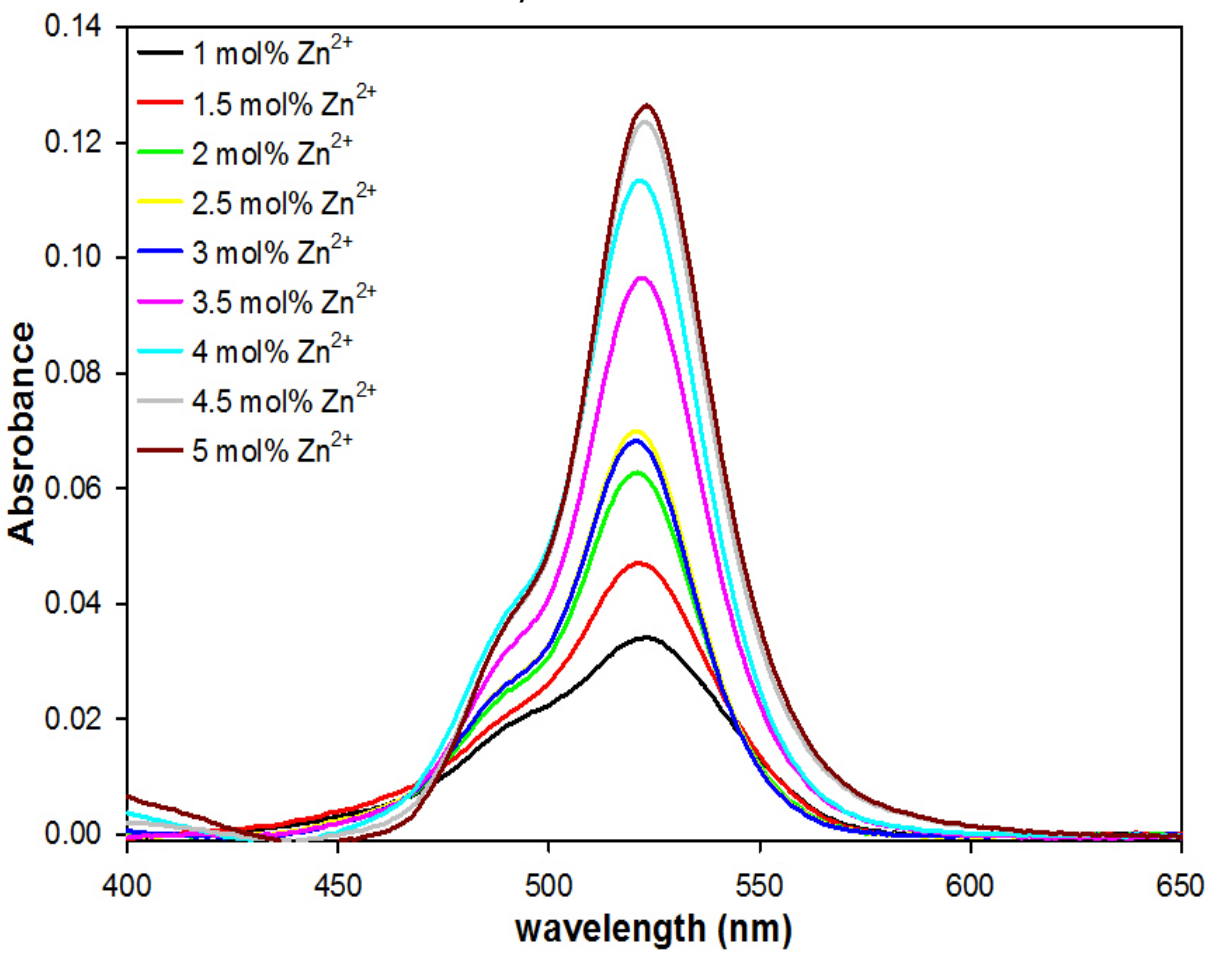


S12: Absorbance Spectrum of $9 \times 10^{-4}$ M Rh6G Deposition Solution Doped PVDF $/ \mathbf{Z n}^{2+}$ Substrate.

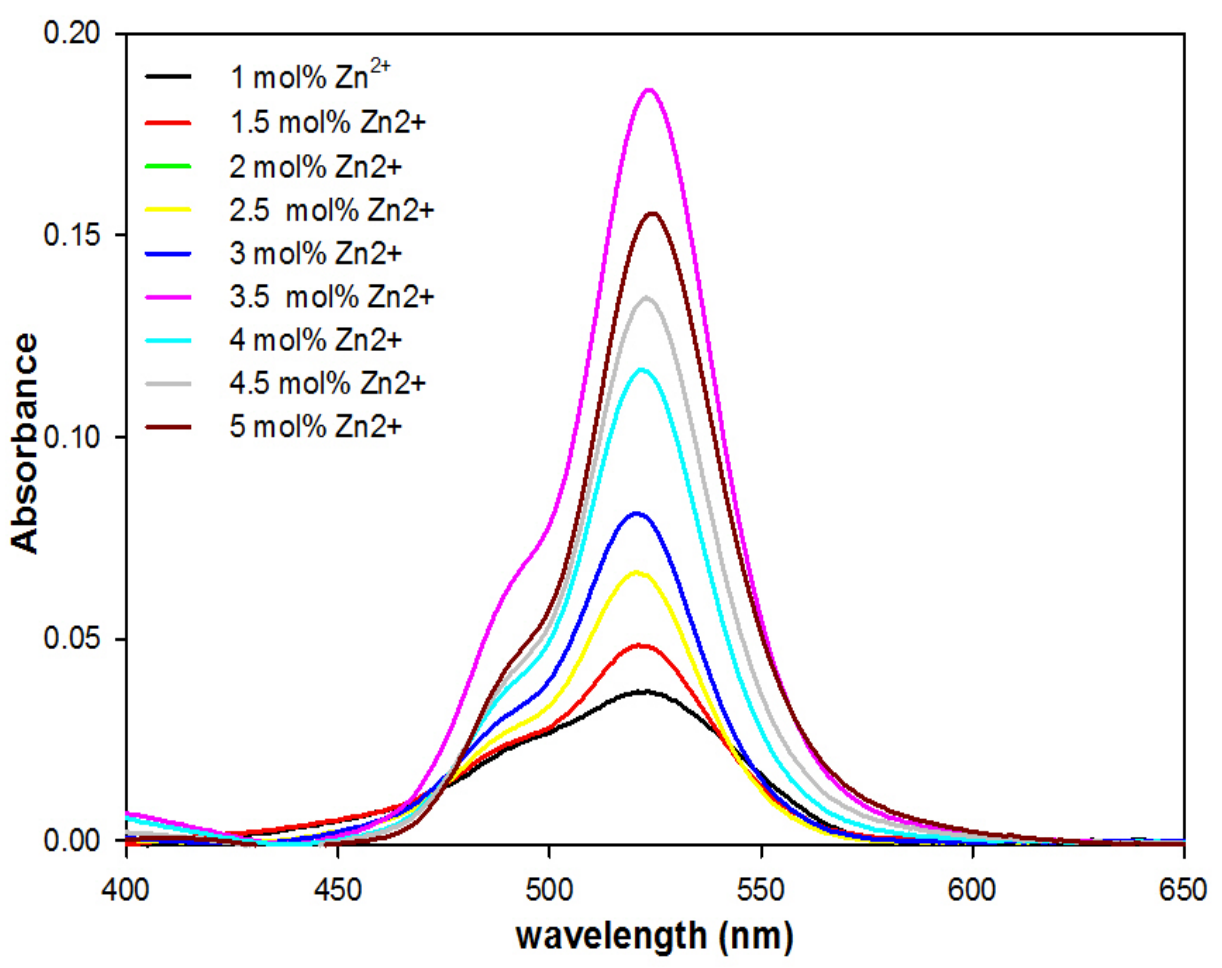

S13: Emission Spectrum of Rhodamine 6G on Glass Substrate.

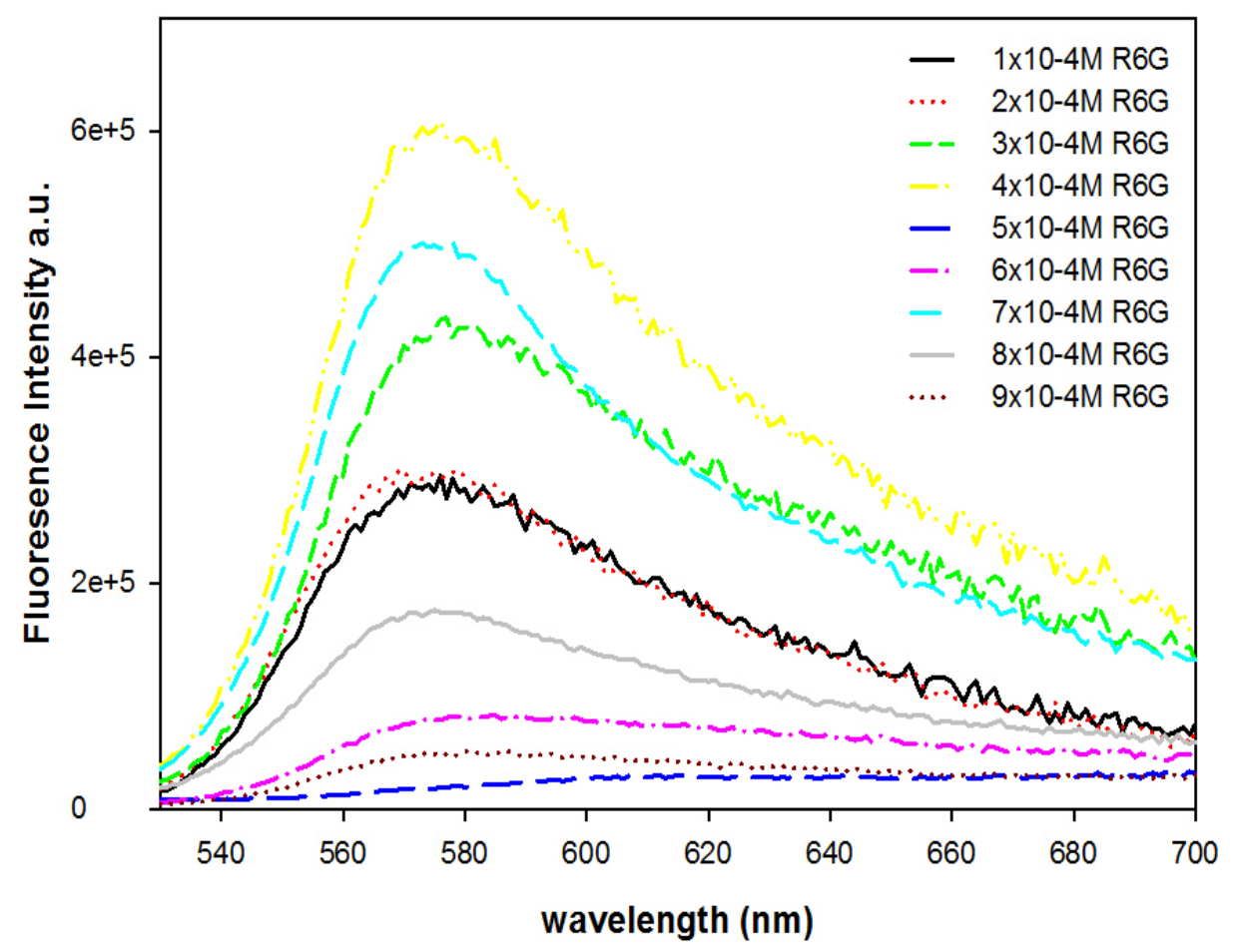


S14: Emission Spectrum of $1 \times 10^{-4}$ M Rh6G Deposition Solution Doped PVDF $/ \mathrm{Zn}^{2+}$ Substrate.

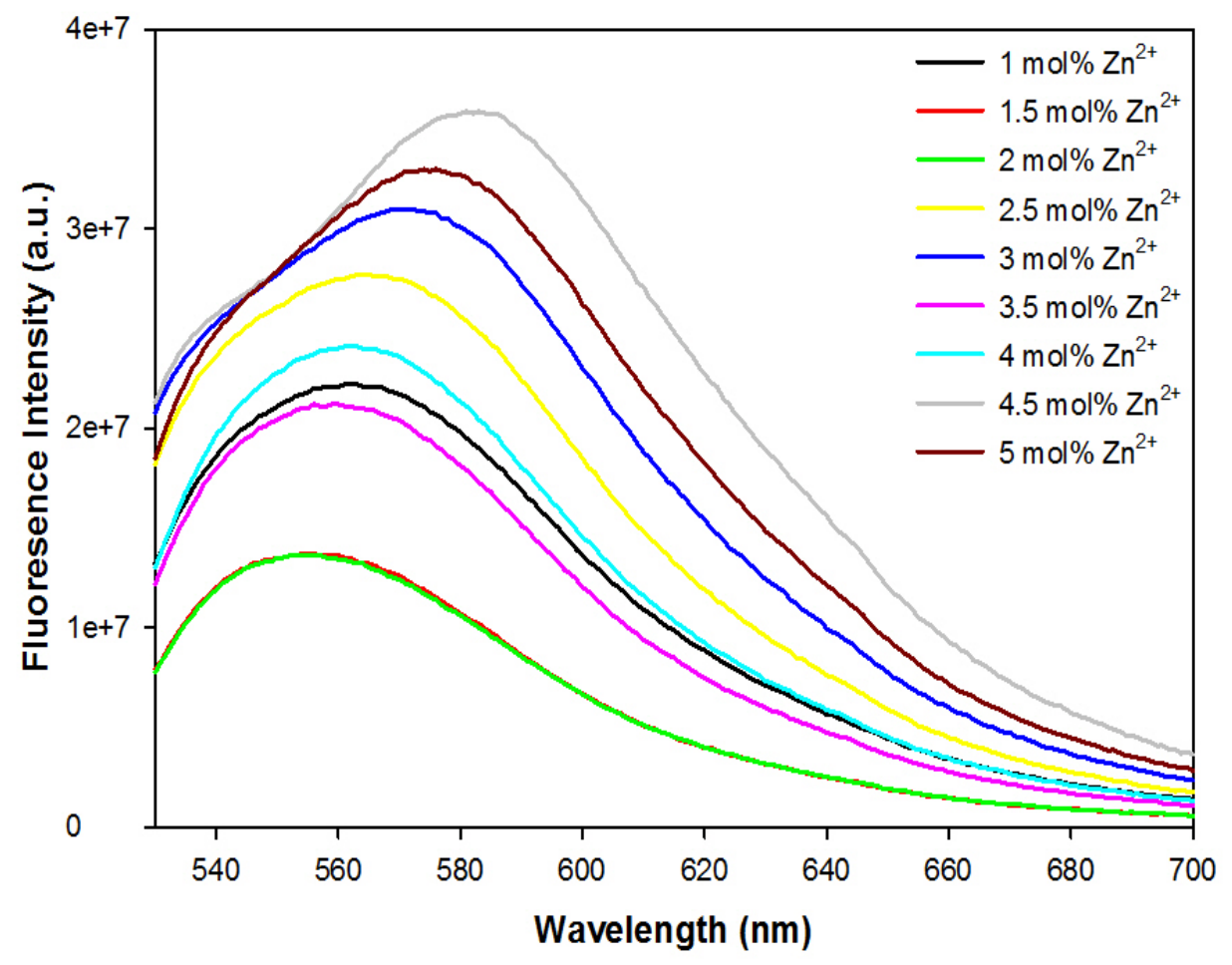

S15: Emission Spectrum of $2 \times 10^{-4}$ M Rh6G Deposition Solution Doped PVDF $/ \mathrm{Zn}^{2+}$ Substrate.

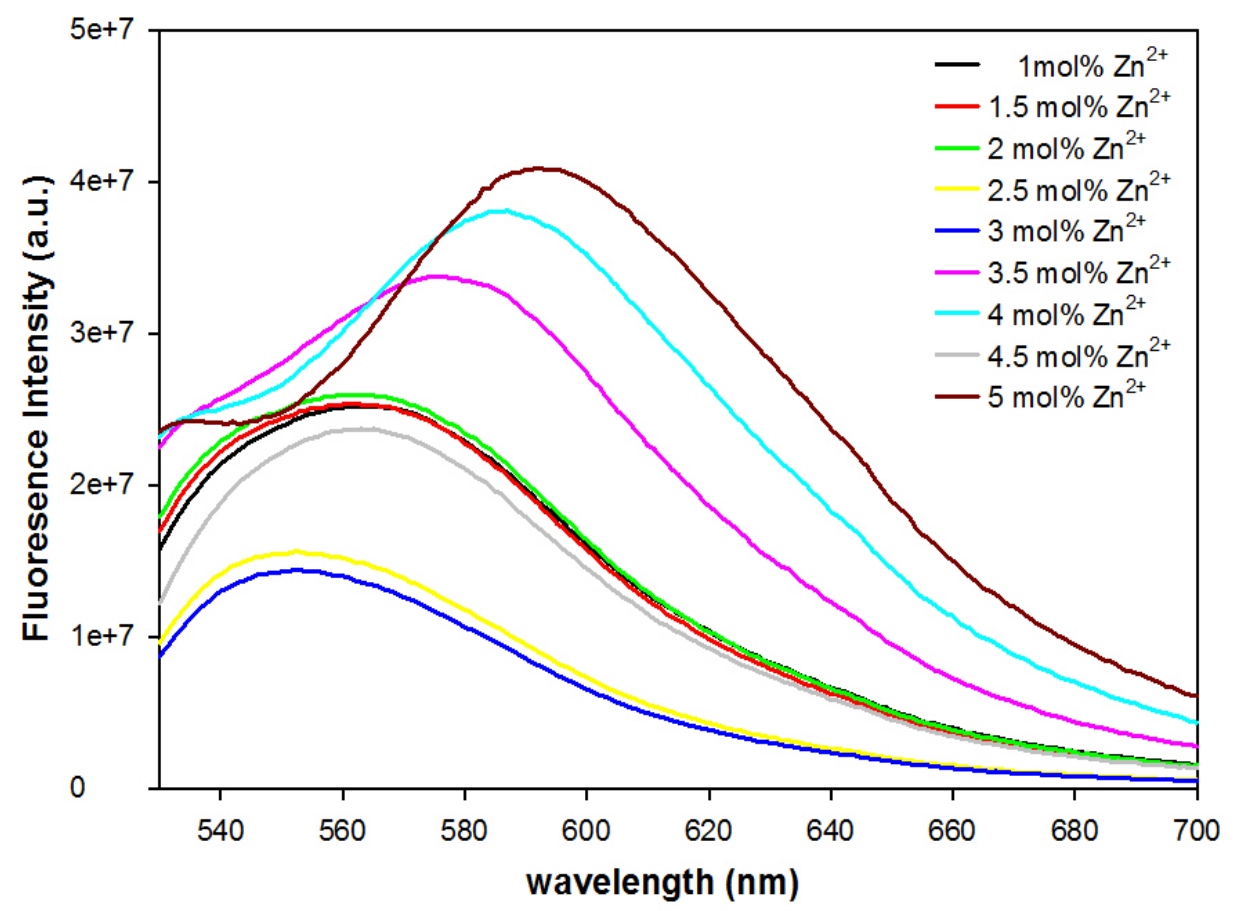


S16: Emission Spectrum of $3 \times 10^{-4}$ M Rh6G Deposition Solution Doped PVDF $/ \mathrm{Zn}^{2+}$ Substrate.

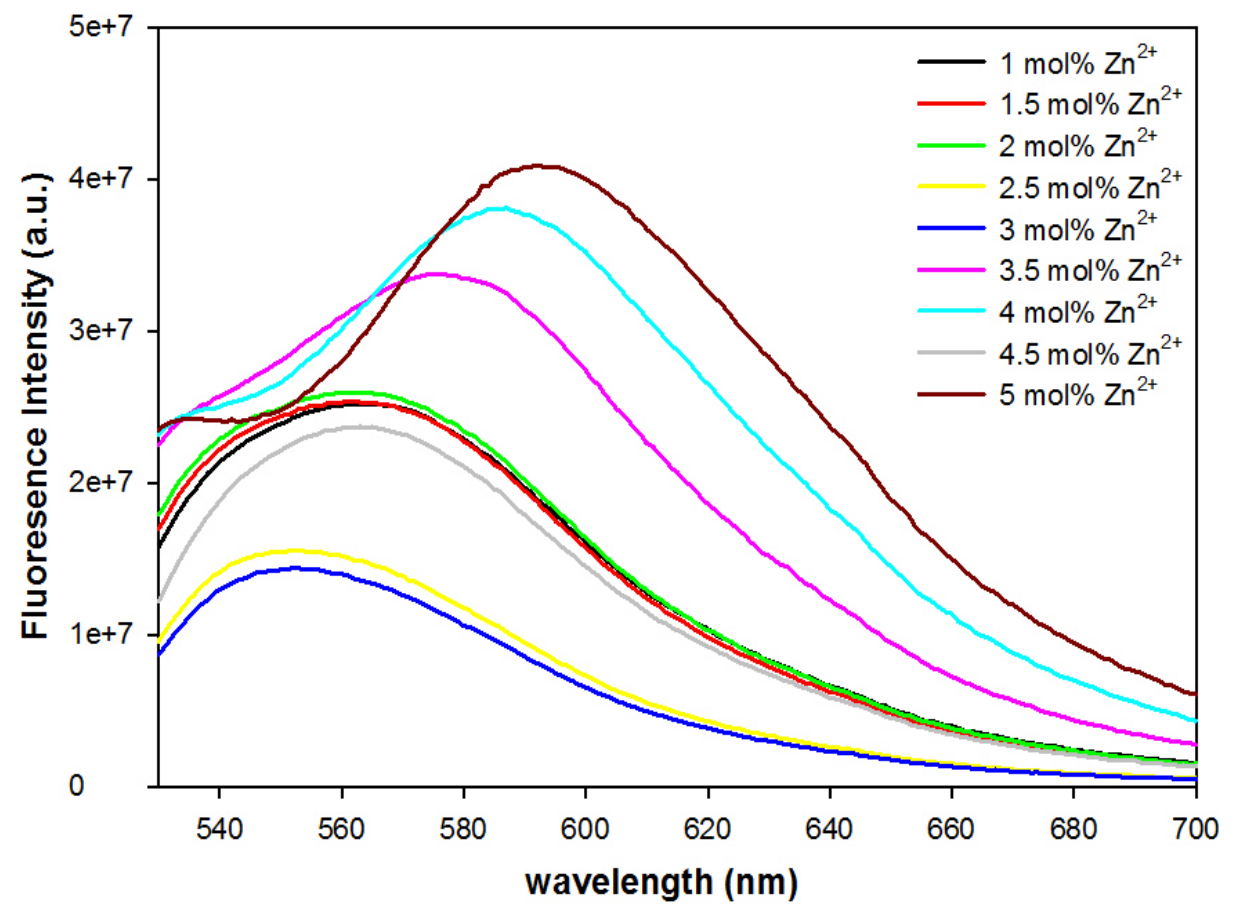

S17: Emission Spectrum of $4 \times 10^{-4}$ M Rh6G Deposition Solution Doped PVDF $/ Z^{2+}$ Substrate.

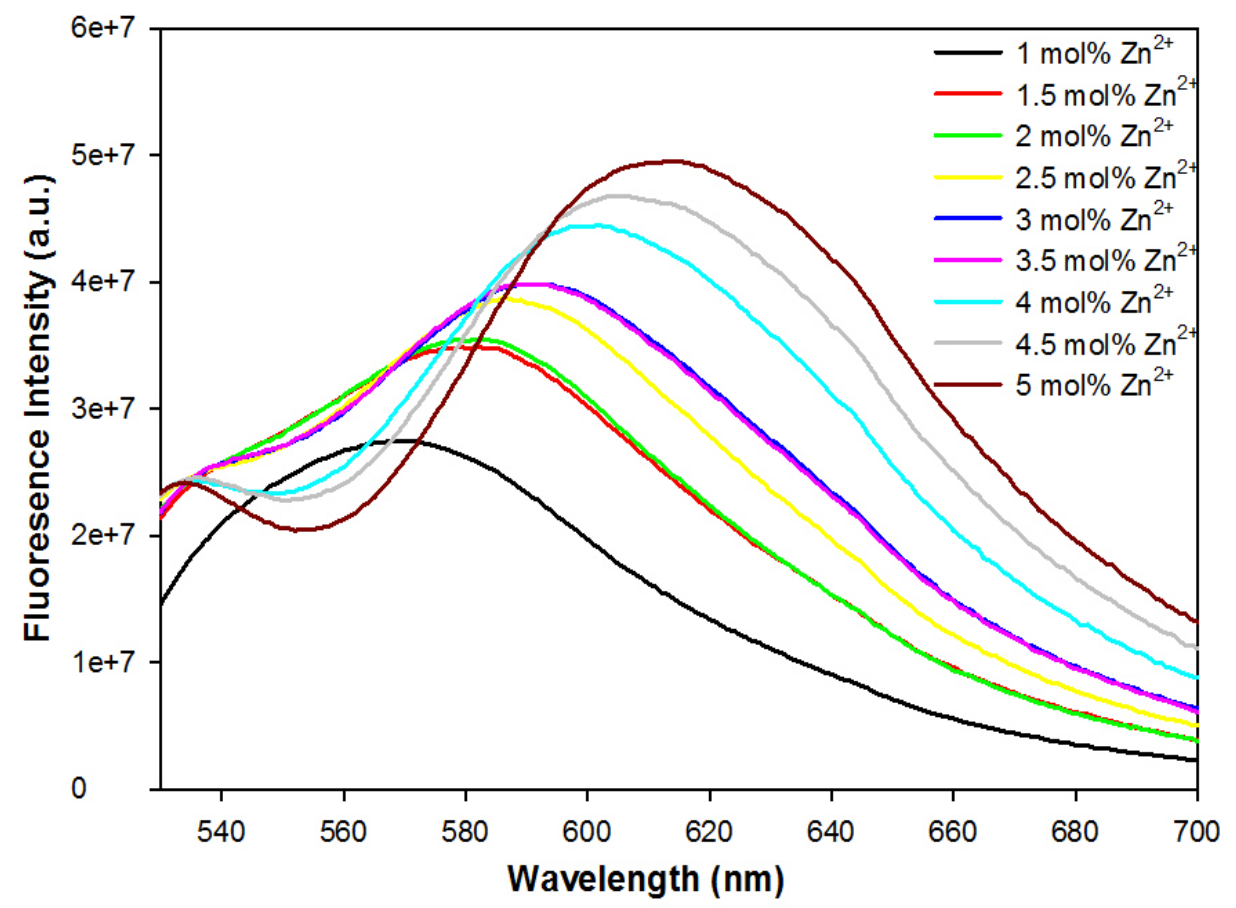


S18: Emission Spectrum of $5 \times 10^{-4}$ M Rh6G Deposition Solution Doped PVDF $/ \mathbf{Z n}^{2+}$ Substrate.

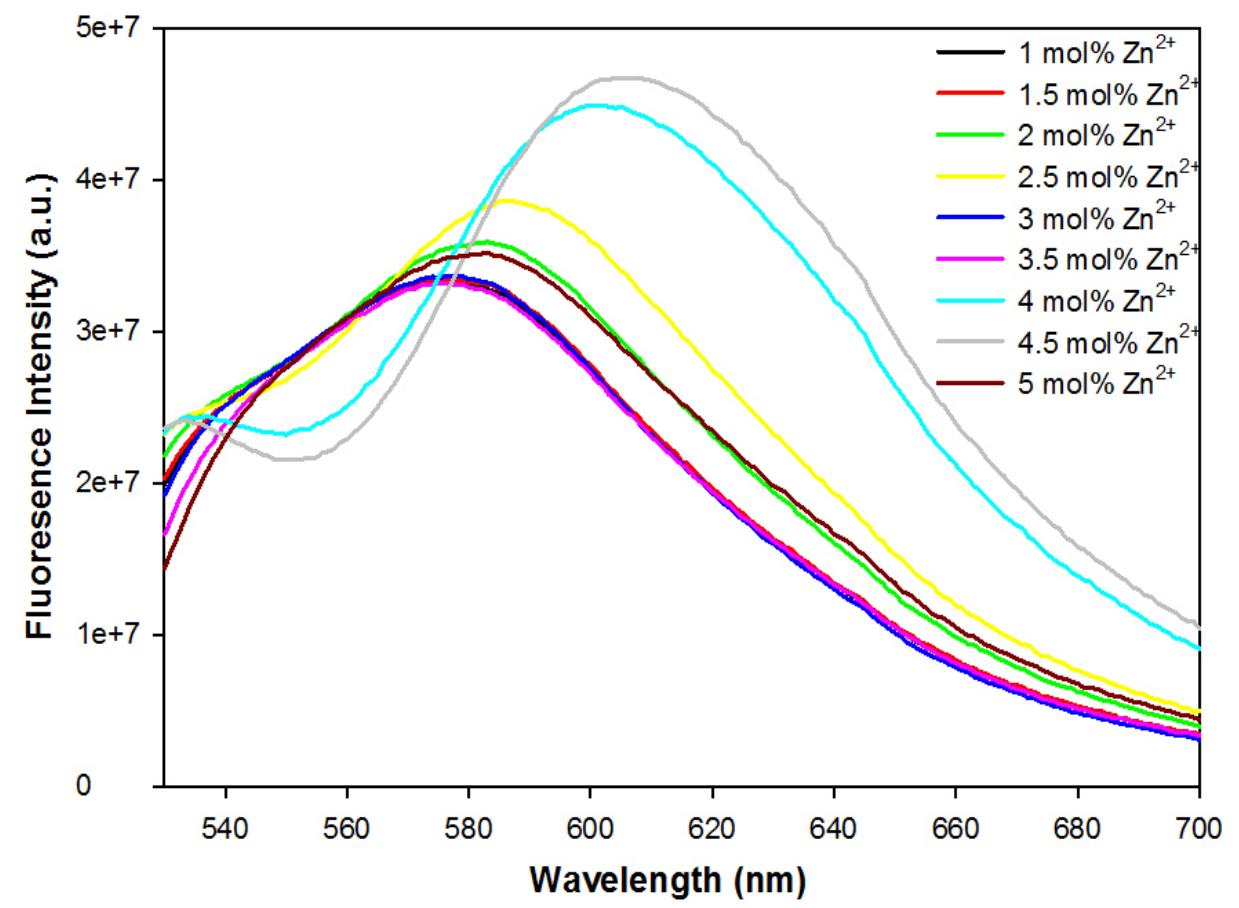

S19: Emission Spectrum of $6 \times 10^{-4}$ M Rh6G Deposition Solution Doped PVDF $/ Z^{2+}$ Substrate.

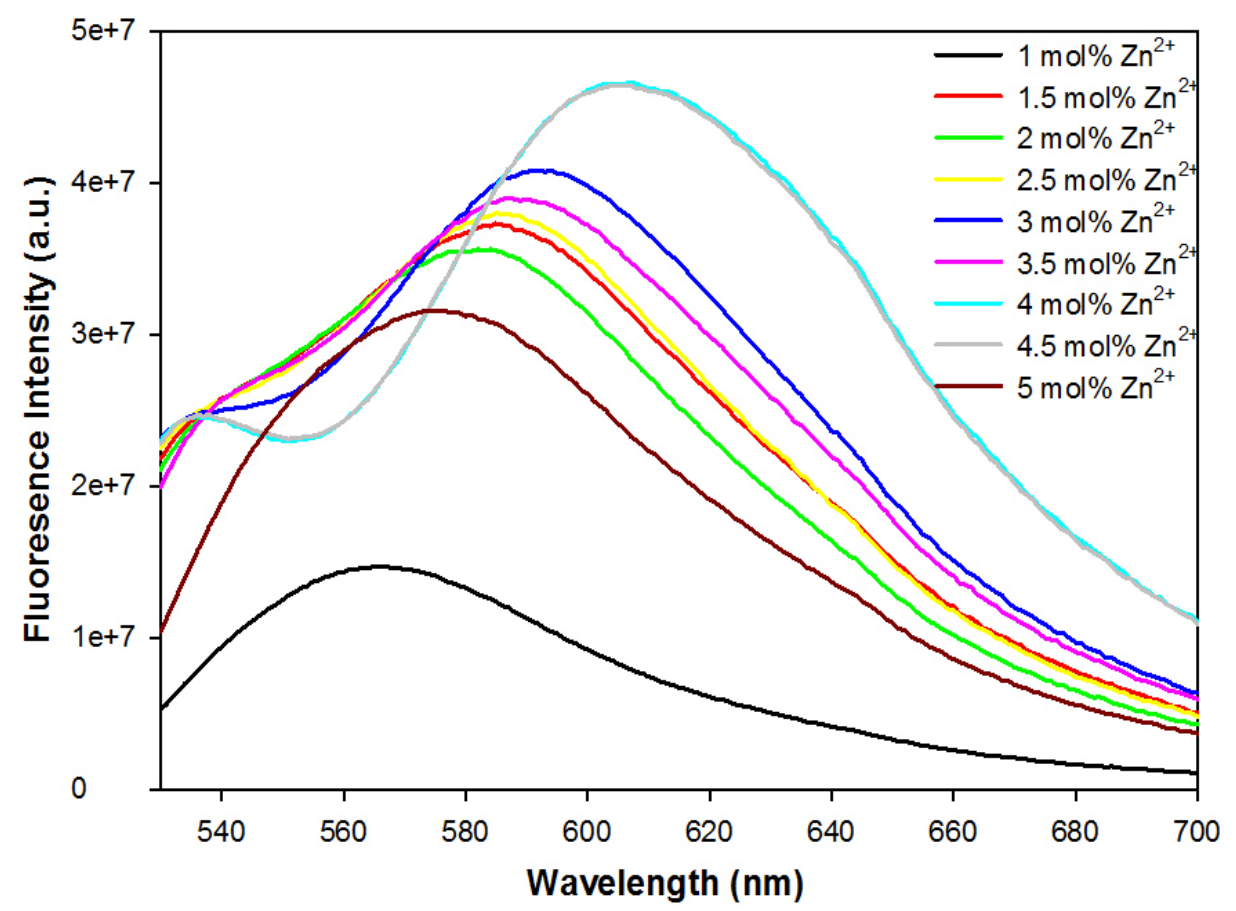


S20: Emission Spectrum of $7 \times 10^{-4}$ M Rh6G Deposition Solution Doped PVDF $/ \mathrm{Zn}^{2+}$ Substrate.

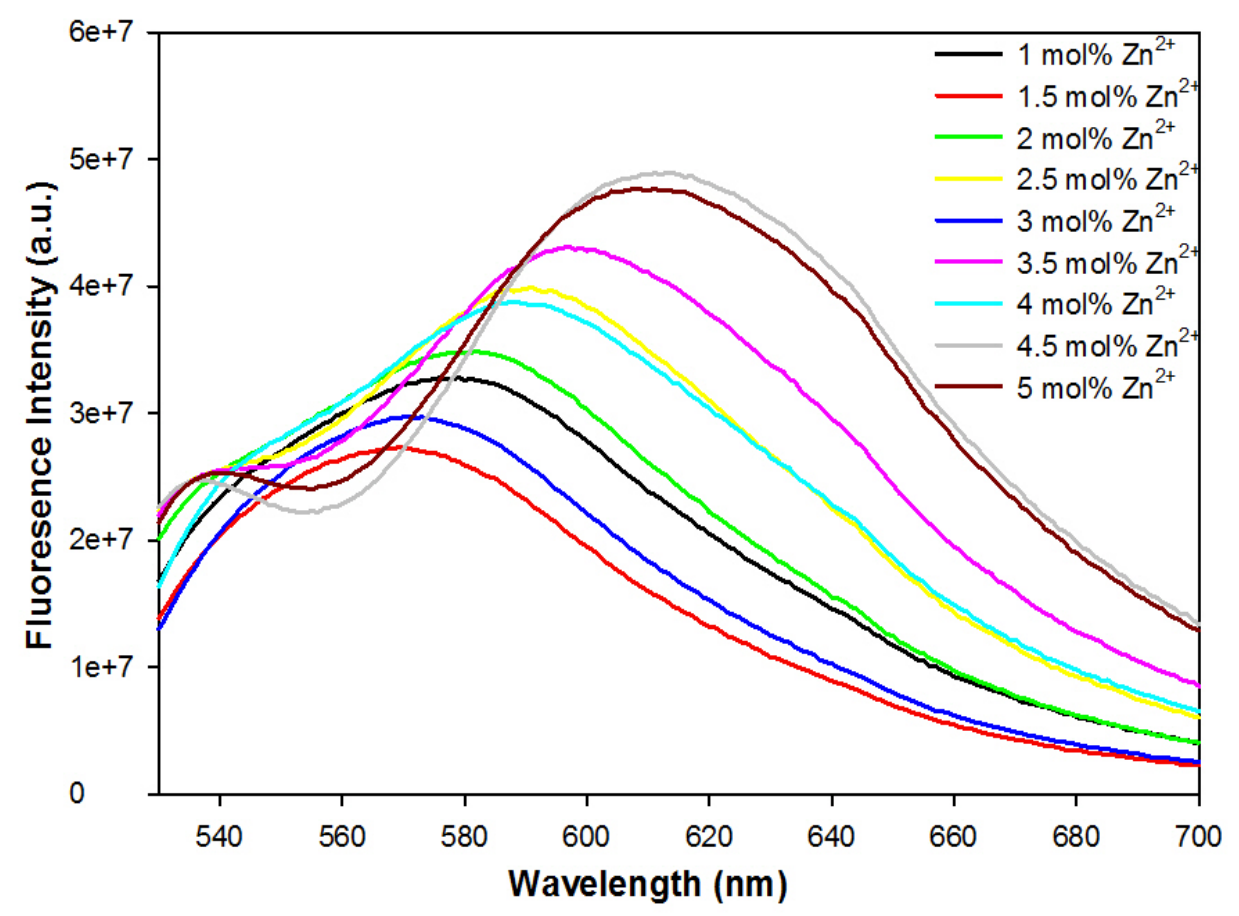

S21: Emission Spectrum of $8 \times 10^{-4}$ M Rh6G Deposition Solution Doped PVDF $/ Z^{2+}$ Substrate.

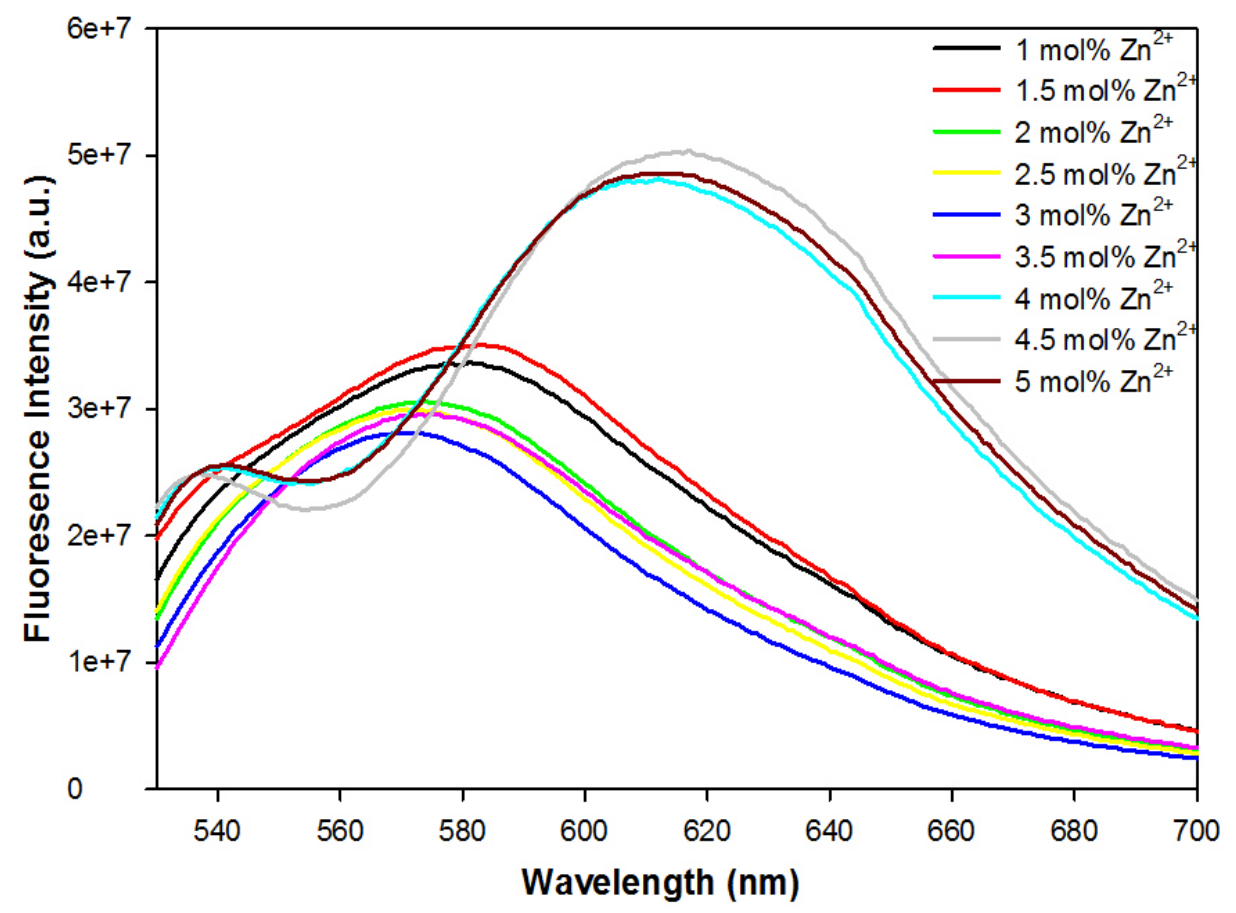


S22: Emission Spectrum of $9 \times 10^{-4}$ M Rh6G Deposition Solution Doped PVDF $/ \mathrm{Zn}^{2+}$ Substrate.

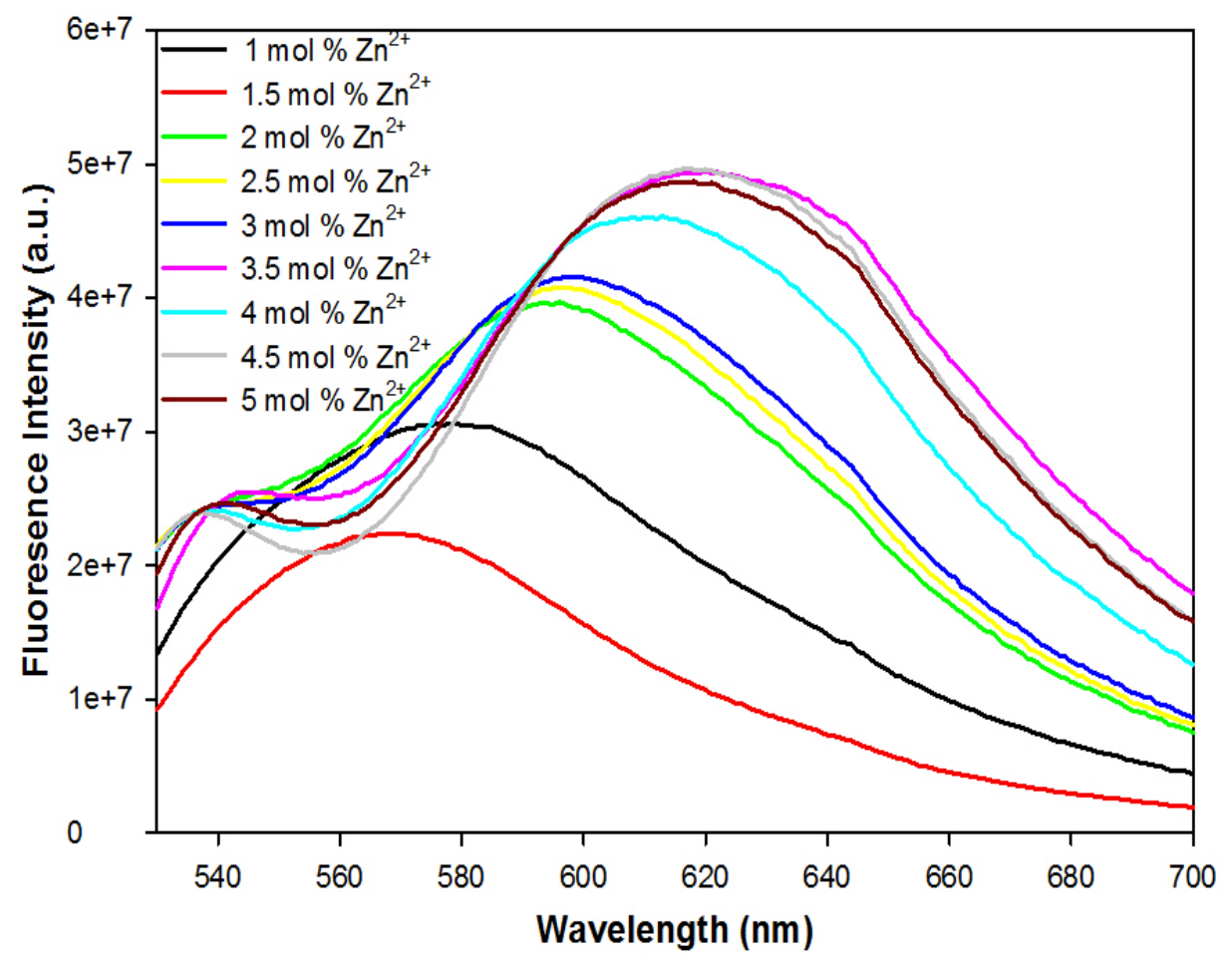

S23: 3D Plot of Absorbance Spectrum of [Rh6G] Doped PVDF/Zn ${ }^{2+}$ Substrate.

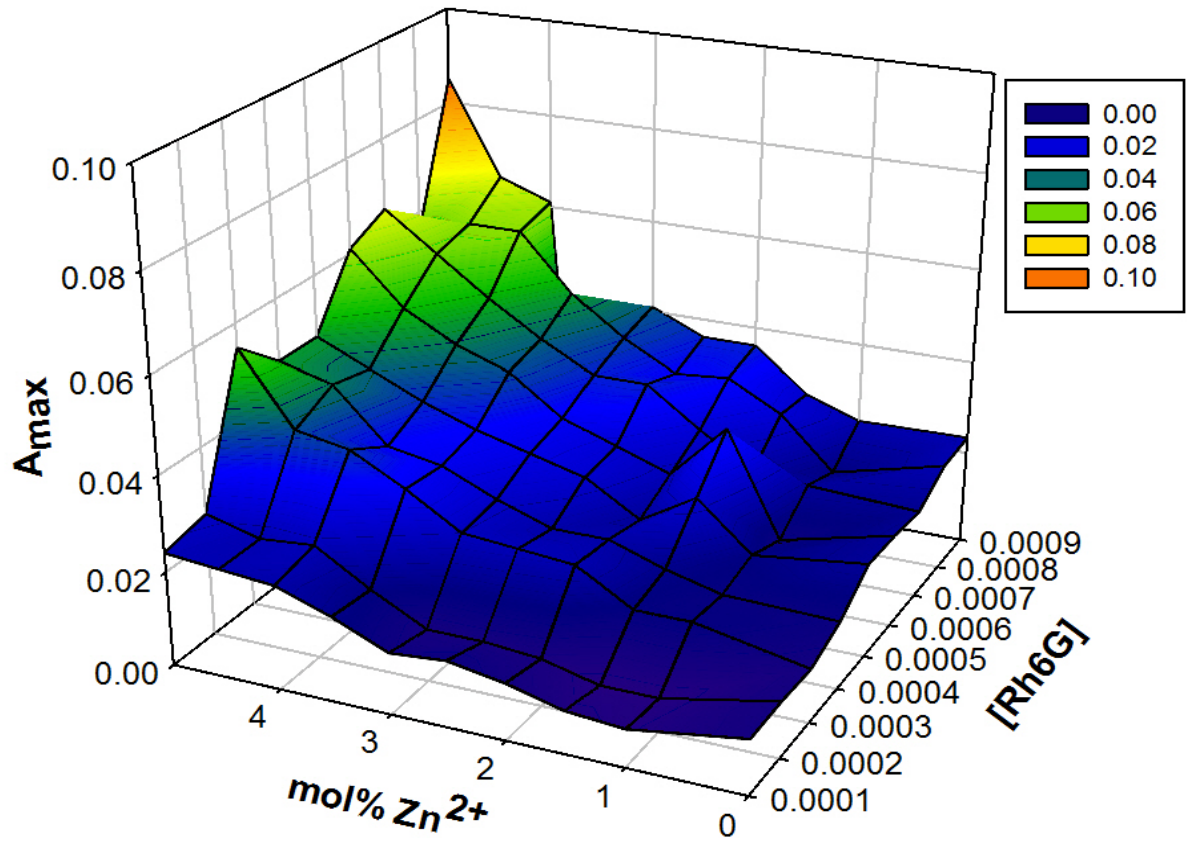




\begin{tabular}{|c|c|c|c|c|c|c|c|c|c|c|}
\hline & $\begin{array}{l}\text { ण } \\
\text { ơ }\end{array}$ & $\begin{array}{l}\text { ूू } \\
\text { ूุ }\end{array}$ & $\begin{array}{l}\text { \%̆ } \\
\text { مุ }\end{array}$ & 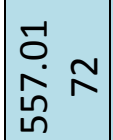 & 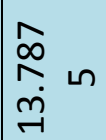 & 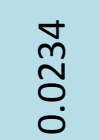 & مَّ & 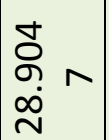 & $\begin{array}{l}0 \\
6 \\
\text { no } \\
0 \\
0\end{array}$ & $\begin{array}{l}\text { フ̊ } \\
8 \\
0\end{array}$ \\
\hline & $\begin{array}{l}0 \\
\qquad \\
\mathbb{\infty} \\
\infty\end{array}$ & $\begin{array}{l}\stackrel{\infty}{\circ} \\
\text { ọ }\end{array}$ & જิ & $\begin{array}{l}\dot{v} \\
\text { ஸ் } \\
\text { ஸn }\end{array}$ & 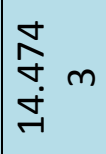 & $\begin{array}{l}\stackrel{\infty}{N} \\
\stackrel{0}{0} \\
0\end{array}$ & 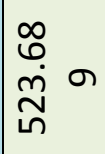 & $\begin{array}{l}\stackrel{\infty}{N} \\
\underset{\infty}{\sim} \\
\stackrel{N}{N}\end{array}$ & 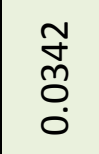 & 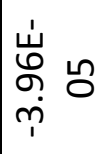 \\
\hline & 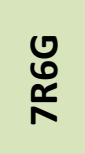 & $\begin{array}{l}\stackrel{\infty}{\%} \\
\text { ọ }\end{array}$ & $\begin{array}{l}\text { \%̆ } \\
\text { ू̆ } \\
0\end{array}$ & 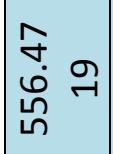 & $\begin{array}{l}\text { ๙ે } \\
\text { ભે }\end{array}$ & 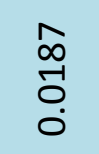 & $\begin{array}{l}m \\
\text { r్ } \\
\stackrel{\text { N }}{n}\end{array}$ & $\begin{array}{l}8 \\
\text { ọ } \\
\text { N }\end{array}$ & $\begin{array}{l}\text { ஸึ } \\
\text { ○ิ } \\
0\end{array}$ & $\begin{array}{l}-1 \\
8 \\
8 \\
0\end{array}$ \\
\hline & $\begin{array}{l}0 \\
0 \\
0 \\
0\end{array}$ & $\begin{array}{l}\infty \\
\text { \%̆ } \\
\text { مุ }\end{array}$ & $\begin{array}{l}\text { ஜू } \\
\text { ू̆ }\end{array}$ & $\begin{array}{ll}\infty & \\
\infty & \circ \\
\text { N่ } & \infty \\
\text { เn } & \end{array}$ & $\begin{array}{l}\mathcal{O} \\
\infty \\
\infty \\
\dot{J}\end{array}$ & \begin{tabular}{l}
0 \\
\multirow{-}{\circ}{} \\
0 \\
0
\end{tabular} & $\begin{array}{l}\stackrel{+}{r} \\
\stackrel{\text { N }}{n}\end{array}$ & $\underset{\substack{N \\
N}}{\infty}$ & 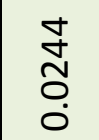 & 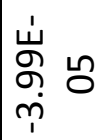 \\
\hline & 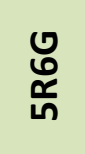 & $\begin{array}{l}\stackrel{\infty}{\circ ̆ ~} \\
\text { ŏ }\end{array}$ & $\begin{array}{l}\text { ğ } \\
\text { ू̆ } \\
0\end{array}$ & 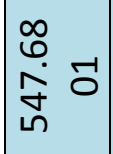 & 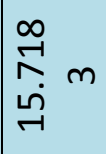 & $\begin{array}{l}\text { बे } \\
\text { ने } \\
\text { ㅇ. }\end{array}$ & 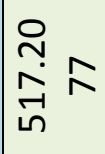 & $\begin{array}{l}\dot{0} \\
\stackrel{\infty}{+} \\
\dot{v}\end{array}$ & $\begin{array}{l}0 \\
\stackrel{\Xi}{~} \\
0 \\
0\end{array}$ & ڤ \\
\hline$\left|\begin{array}{l|}n \\
\tilde{n} \\
\frac{0}{0} \\
c \\
0\end{array}\right|$ & 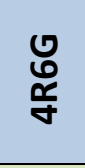 & कू & $\begin{array}{l}\text { ஜू } \\
\text { ू̆ } \\
\text { ó }\end{array}$ & 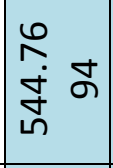 & $\underset{m}{\text { m }} m$ & $\begin{array}{l}\stackrel{m}{\stackrel{2}{-}} \\
\stackrel{0}{0}\end{array}$ & $\begin{array}{l}\text { ㄱ } \\
\text { ผี่ } \\
\text { ஸ่ }\end{array}$ & 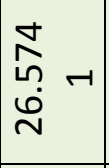 & $\begin{array}{l}\stackrel{-1}{N} \\
\stackrel{-}{0} \\
0\end{array}$ & 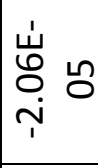 \\
\hline 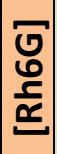 & 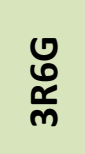 & ஸू & $\begin{array}{l}\text { รू. } \\
\text { Оุ }\end{array}$ & $\begin{array}{l}\stackrel{0}{m} \\
\text { in } \\
m \\
\text { ஸn }\end{array}$ & $\stackrel{\stackrel{n}{N}}{\stackrel{\sim}{r}}+$ & $\begin{array}{l}\stackrel{m}{7} \\
\stackrel{-}{\circ} \\
\stackrel{0}{0}\end{array}$ & $\stackrel{N}{\stackrel{\infty}{0}} \underset{\sim}{\sim} \stackrel{\infty}{\sim}$ & $\begin{array}{l}\widetilde{O} \\
\text { m } \\
\stackrel{\omega}{N}\end{array}$ & ণ্' & 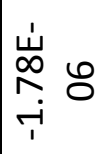 \\
\hline & 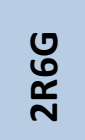 & $\begin{array}{l}\text { \%̆ } \\
\text { ুু }\end{array}$ & 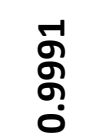 & 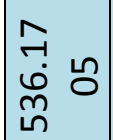 & \begin{tabular}{ll}
$\stackrel{0}{8}$ & \\
\multirow{8}{*}{} & 0 \\
$\dot{J}$ &
\end{tabular} & $\begin{array}{l}\infty \\
\stackrel{\infty}{-} \\
\stackrel{\sigma}{0} \\
\stackrel{0}{0}\end{array}$ & 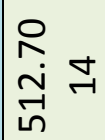 & 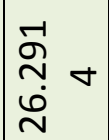 & \begin{tabular}{l}
\multirow{2}{0}{} \\
$\stackrel{1}{0}$ \\
$\stackrel{0}{0}$
\end{tabular} & 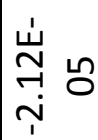 \\
\hline & 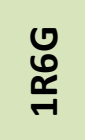 & $\begin{array}{l}\text { \%̆ } \\
\text { مุ }\end{array}$ & $\begin{array}{l}\text { ने } \\
\text { જ̆ } \\
\text { مٌ }\end{array}$ & 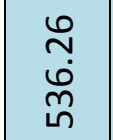 & $\underset{\stackrel{r}{N}}{\underset{j}{\sim}} \sigma$ & $\begin{array}{l}0 \\
ન \\
\stackrel{-}{0} \\
0\end{array}$ & 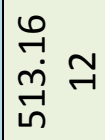 & $\begin{array}{l}\text { m} \\
m \\
\dot{m} \\
\stackrel{v}{*}\end{array}$ & $\begin{array}{l}0 \\
0 \\
0 \\
0 \\
0\end{array}$ & 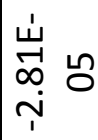 \\
\hline & $\frac{\overline{0}}{\frac{\pi}{\frac{\pi}{2}}} y$ & $\simeq$ & $\begin{array}{l}\grave{ত} \\
\stackrel{\text { n}}{ }\end{array}$ & 3 & 드 & 가 & $\stackrel{N}{3}$ & ح & స్ & 인 \\
\hline & 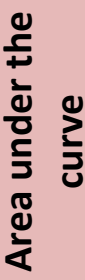 & 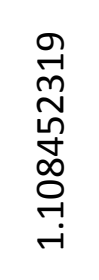 & 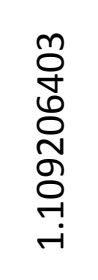 & 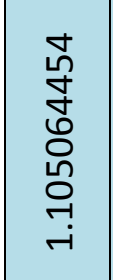 & 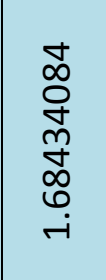 & 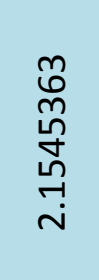 & 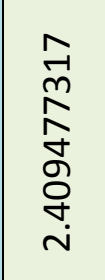 & 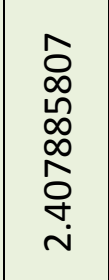 & 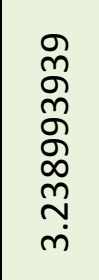 & 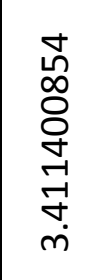 \\
\hline & & 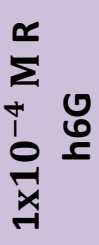 & 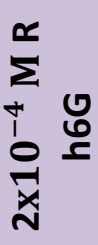 & $\mid \begin{array}{ll}\simeq & \\
\Sigma & \\
1 & 0 \\
1 & 0 \\
0 & c \\
\vec{x} & \\
m & \end{array}$ & 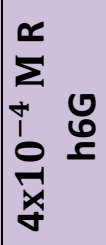 & 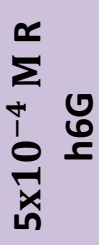 & 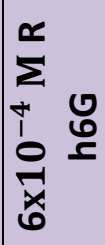 & 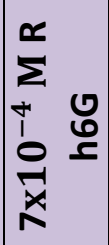 & 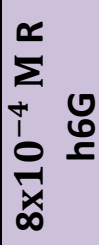 & 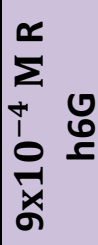 \\
\hline
\end{tabular}




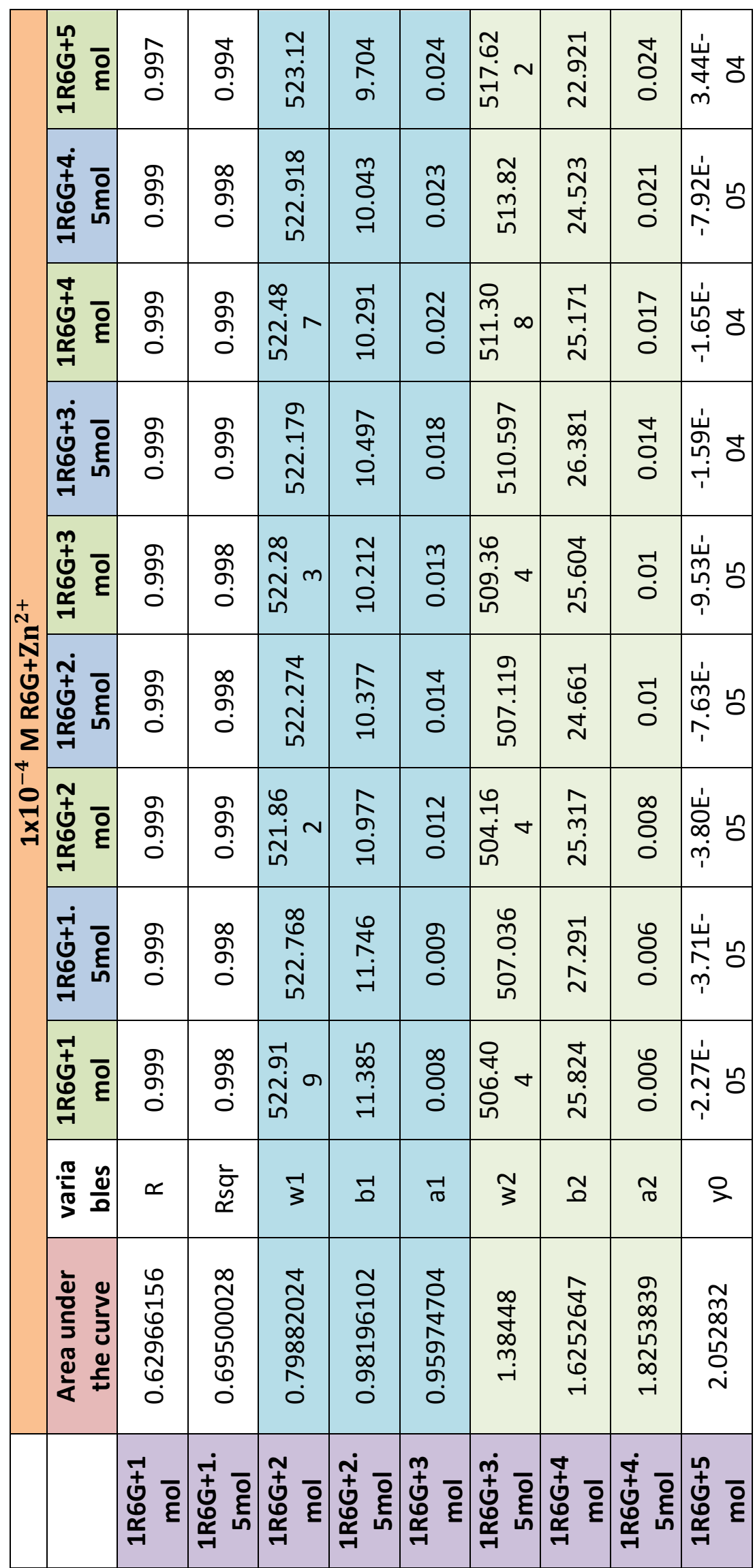




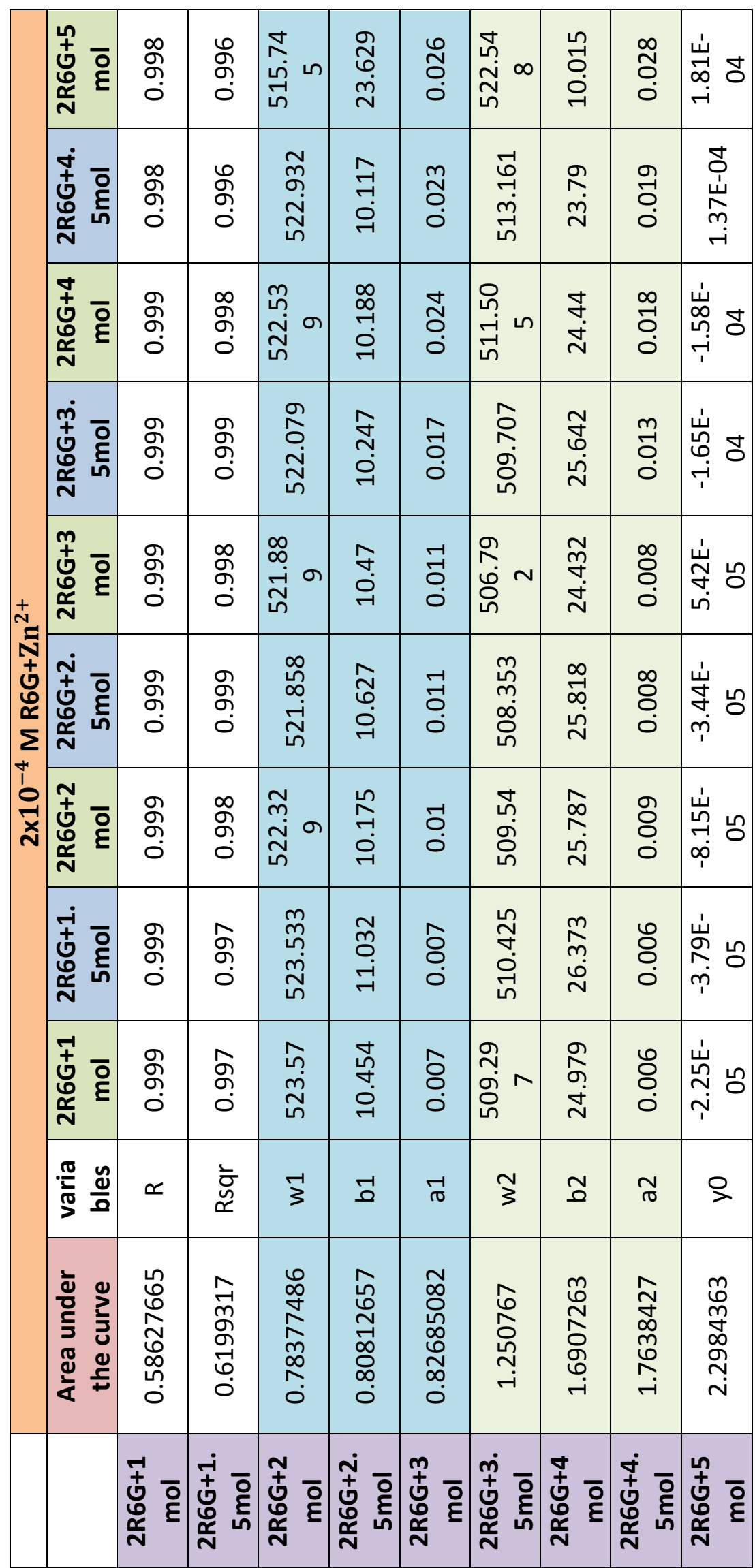




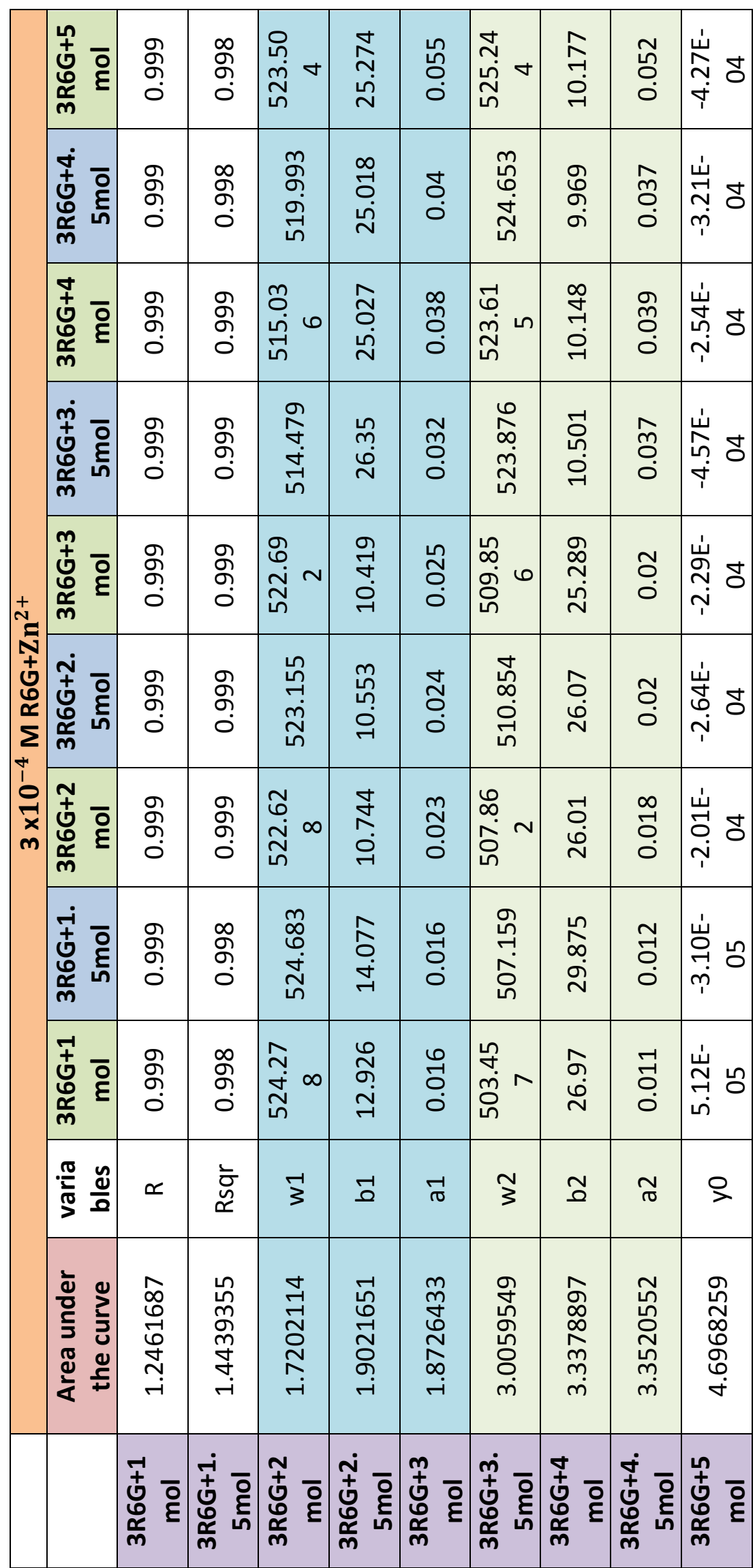




\begin{tabular}{|c|c|c|c|c|c|c|c|c|c|c|}
\hline & 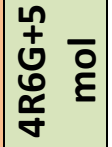 & $\begin{array}{l}\infty \\
\text { مุ } \\
0\end{array}$ & बे & 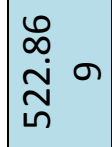 & $\begin{array}{l}\text { సे } \\
\text { ন }\end{array}$ & 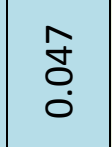 & $\begin{array}{l}\text { ก } \\
\text { ป் } \\
\text { ஸे }\end{array}$ & $\begin{array}{l}\text { शे } \\
\text { बं }\end{array}$ & $\begin{array}{l}\stackrel{N}{\mathcal{O}} \\
\stackrel{0}{0}\end{array}$ & 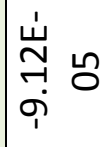 \\
\hline & 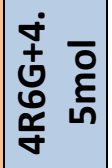 & $\begin{array}{l}\text { ने } \\
\text { ळே }\end{array}$ & $\begin{array}{l}\text { مे } \\
\text { مே }\end{array}$ & $\begin{array}{l}\stackrel{0}{N} \\
\text { ஸ் } \\
\text { กิ }\end{array}$ & $\begin{array}{l}\text { D } \\
\text { ơ } \\
\dot{\sim}\end{array}$ & 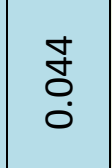 & 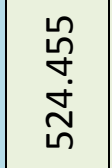 & 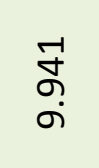 & $\begin{array}{l}\text { \& } \\
\text { ல }\end{array}$ & 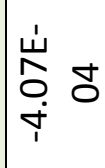 \\
\hline & 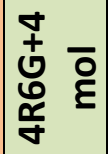 & बे & $\begin{array}{l}\infty \\
\text { } \\
\text { ○े }\end{array}$ & 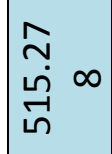 & 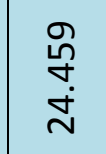 & $\begin{array}{l}m \\
\stackrel{m}{\circ} \\
\stackrel{0}{0}\end{array}$ & $\begin{array}{l}\stackrel{0}{0} \\
\stackrel{N}{N}\end{array}$ & $\begin{array}{l}\stackrel{\infty}{-} \\
\stackrel{+}{+} \\
\stackrel{-}{-1}\end{array}$ & $\begin{array}{l}0 \\
\text { mo } \\
0 \\
0\end{array}$ & 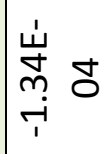 \\
\hline & 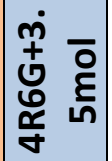 & बे & बे & 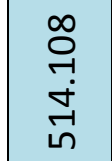 & $\begin{array}{l}\stackrel{v}{v} \\
\stackrel{\leftrightarrow}{\mathscr{v}}\end{array}$ & $\begin{array}{l}\text { ஜூ } \\
0 \\
0\end{array}$ & $\begin{array}{l}\text { ठ } \\
\qquad \\
\ddot{N} \\
\text { ஸे }\end{array}$ & $\begin{array}{l}\text { N } \\
\text { ஸ̊ } \\
\text { กิ }\end{array}$ & $\begin{array}{l}\text { r } \\
\text { Ọ } \\
0\end{array}$ & $\begin{array}{l}\dot{w} \\
\infty \\
\infty \\
m \\
1\end{array}$ \\
\hline$\stackrel{+}{\sim}$ & 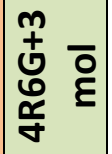 & $\begin{array}{l}\text { ने } \\
\text { Оे }\end{array}$ & $\begin{array}{l}\infty \\
\text { مे } \\
0\end{array}$ & $\begin{array}{l}\text { హె } \\
\text { กู }\end{array}$ & $\begin{array}{l}\vec{m} \\
m \\
\dot{\sim}\end{array}$ & $\begin{array}{l}\stackrel{0}{N} \\
\text { Oे. }\end{array}$ & 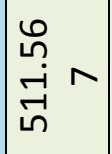 & $\begin{array}{l}\overrightarrow{1} \\
\infty \\
\dot{d}\end{array}$ & $\begin{array}{l}\text { ָे } \\
\text { Oீ. }\end{array}$ & 岂 \\
\hline $\begin{array}{l}1 \\
0 \\
0 \\
0 \\
\Sigma \\
\Sigma\end{array}$ & 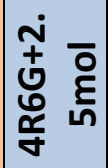 & $\begin{array}{l}\text { ने } \\
\text { Оุ }\end{array}$ & $\begin{array}{l}\text { बे } \\
\text { مே }\end{array}$ & 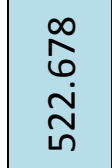 & 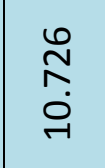 & $\begin{array}{l}\text { Ð઼ } \\
\text { Oे }\end{array}$ & \begin{tabular}{l}
\multirow{N}{*}{} \\
$\infty$ \\
$\infty$ \\
0 \\
\end{tabular} & $\begin{array}{l}\infty \\
\infty \\
\text { ஸे } \\
\text { N }\end{array}$ & 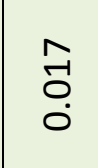 & 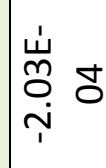 \\
\hline $\begin{array}{l}1 \\
\frac{1}{x} \\
y \\
y\end{array}$ & 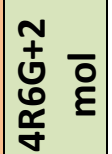 & बू & $\begin{array}{l}\text { ने } \\
\text { Оே }\end{array}$ & $\begin{array}{l}\text { Ln } \\
\text { N } \\
\text { Nิ }\end{array}$ & 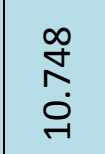 & $\begin{array}{l}\text { స్ } \\
\text { ல }\end{array}$ & 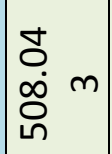 & $\begin{array}{l}\infty \\
\infty \\
\varphi \\
\stackrel{\omega}{N}\end{array}$ & $\begin{array}{l}\text { न्न } \\
0 \\
0\end{array}$ & 岕 \\
\hline & 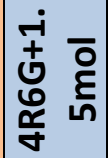 & $\begin{array}{l}\infty \\
\text { ऴ } \\
0\end{array}$ & बे & 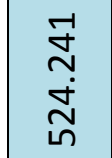 & 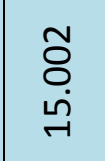 & $\begin{array}{l}\infty \\
\stackrel{1}{0} \\
0 \\
0\end{array}$ & 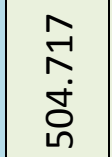 & $\begin{array}{l}\stackrel{n}{\mathcal{H}} \\
\stackrel{j}{m}\end{array}$ & $\begin{array}{l}\text { 궁 } \\
\text { ○’ }\end{array}$ & 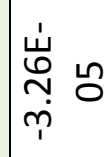 \\
\hline & 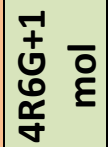 & बे & $\begin{array}{l}\text { مे } \\
\text { مே }\end{array}$ & $\begin{array}{l}\stackrel{n}{m} \\
\stackrel{\sim}{\sim}\end{array}$ & 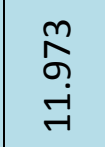 & $\stackrel{n}{\stackrel{n}{0}}$ & 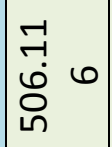 & $\begin{array}{l}\text { กี } \\
\text { กุ } \\
\text { กิ }\end{array}$ & $\begin{array}{l}-1 \\
\stackrel{-}{0} \\
0\end{array}$ & 岂 \\
\hline & $\frac{\pi}{\frac{\pi}{5}} \frac{\tilde{d}}{0}$ & $\simeq$ & $\begin{array}{l}\bar{\vdots} \\
\bar{\sim} \\
\check{\sim}\end{array}$ & 3 & 요 & 가 & $\tilde{3}$ & ح & స్ & $\stackrel{?}{>}$ \\
\hline & 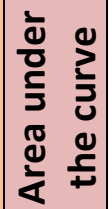 & $\begin{array}{l}\text { ปี } \\
\stackrel{-}{7} \\
\text { ㄱ. } \\
\text { - }\end{array}$ & 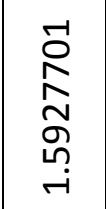 & 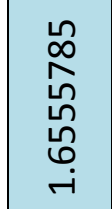 & 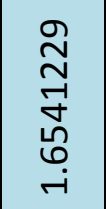 & $\begin{array}{l}\stackrel{-}{o} \\
\stackrel{+}{+} \\
\stackrel{N}{\sigma} \\
\text { i }\end{array}$ & 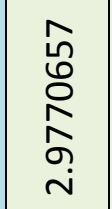 & \begin{tabular}{l}
0 \\
\multirow{f}{*}{} \\
N \\
$\infty$ \\
$\infty$ \\
$i$ \\
$\sim$
\end{tabular} & 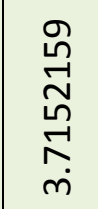 & $\begin{array}{l}\text { ठ } \\
\stackrel{N}{N} \\
\text { ஸू } \\
\text { ஸ் }\end{array}$ \\
\hline & & 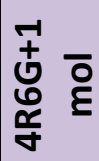 & 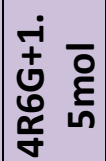 & 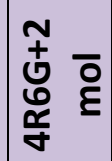 & 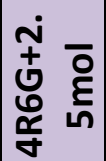 & 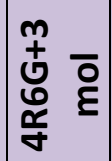 & 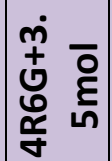 & 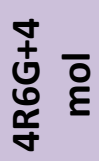 & 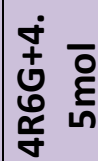 & 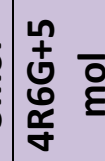 \\
\hline
\end{tabular}




\begin{tabular}{|c|c|c|c|c|c|c|c|c|c|c|}
\hline & 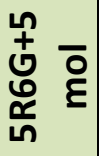 & $\begin{array}{l}\text { Oे } \\
\text { Oे } \\
\text { - }\end{array}$ & $\begin{array}{l}\text { જे } \\
\text { م్ }\end{array}$ & 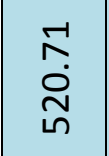 & $\begin{array}{l}\stackrel{\infty}{\sim} \\
\stackrel{\sim}{\sim} \\
\stackrel{\sim}{N}\end{array}$ & $\begin{array}{l}\text { fे } \\
\dot{0}\end{array}$ & 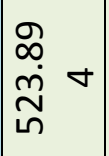 & 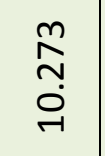 & $\begin{array}{l}\stackrel{\infty}{+} \\
\stackrel{0}{0} \\
\dot{0}\end{array}$ & $\begin{array}{l}\dot{u} \\
\infty \\
\stackrel{\infty}{+} \\
\dot{\gamma}\end{array}$ \\
\hline & 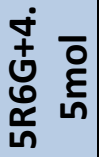 & $\begin{array}{l}\text { Oे } \\
\text { مे } \\
\text { - }\end{array}$ & $\begin{array}{l}\text { o } \\
\text { Oे } \\
\text { ○े }\end{array}$ & 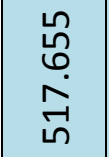 & 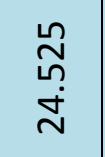 & $\begin{array}{l}\mathcal{Y} \\
\stackrel{0}{0}\end{array}$ & 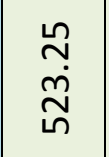 & $\begin{array}{l}\text { नै } \\
\text { ○े }\end{array}$ & $\begin{array}{l} \pm \\
0 \\
0\end{array}$ & $\begin{array}{l}\stackrel{\leftrightarrow}{N} \\
\stackrel{n}{p} \\
r\end{array}$ \\
\hline & 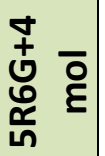 & $\begin{array}{l}\text { হे } \\
\text { Oे } \\
0\end{array}$ & $\begin{array}{l}\text { જे } \\
\text { જ̆ } \\
\text { - }\end{array}$ & $\begin{array}{l}\infty \\
\infty \\
\dot{\vec{j}} \\
\text { जn }\end{array}$ & 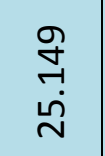 & $\begin{array}{l}\hat{n} \\
0 \\
0\end{array}$ & 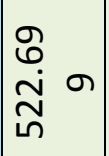 & $\begin{array}{l}\stackrel{0}{N} \\
\text { m} \\
\stackrel{-}{-}\end{array}$ & $\begin{array}{l}\hat{n} \\
0 \\
0\end{array}$ & 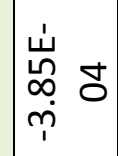 \\
\hline & 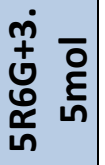 & $\begin{array}{l}\text { gे } \\
\text { Oे. } \\
0\end{array}$ & $\begin{array}{l}\text { शे } \\
\text { Oे } \\
0\end{array}$ & 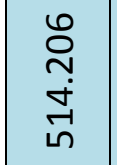 & $\stackrel{\nabla}{\grave{\omega}}$ & $\begin{array}{l}m \\
\tilde{o} \\
0 \\
0\end{array}$ & $\begin{array}{l}\text { Oे } \\
\text { तें } \\
\text { ஸे }\end{array}$ & $\begin{array}{l}\text { Nิ } \\
\text { กิ? } \\
\text { - }\end{array}$ & $\begin{array}{l}\text { Oे } \\
0\end{array}$ & 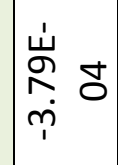 \\
\hline & 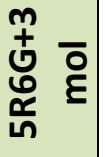 & $r$ & $\begin{array}{l}\text { शे } \\
\text { م్ } \\
\text { - }\end{array}$ & $\begin{array}{l}0 \\
o \\
\text { in } \\
\text { ñ }\end{array}$ & 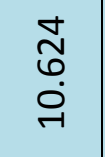 & $\stackrel{m}{0}$ & $\begin{array}{l}\text { o } \\
\text { oi } \\
\text { in }\end{array}$ & $\begin{array}{l}\stackrel{m}{\tilde{\omega}} \\
\stackrel{\omega}{N}\end{array}$ & 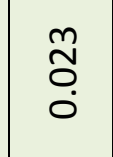 & 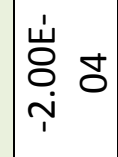 \\
\hline & 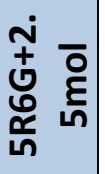 & $\begin{array}{l}\text { शे } \\
\text { Oे } \\
0\end{array}$ & $\begin{array}{l}\text { } \\
\text { Oे } \\
\text { - }\end{array}$ & 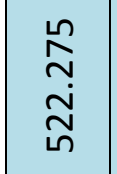 & $\begin{array}{l}0 \\
\tilde{n} \\
0 \\
0 \\
-1\end{array}$ & $\begin{array}{l}\hat{O} \\
0 \\
0\end{array}$ & 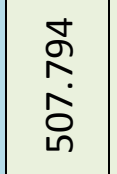 & $\begin{array}{l}\stackrel{\bigsqcup}{0} \\
\stackrel{\leftrightarrow}{\sim}\end{array}$ & 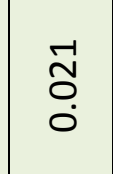 & 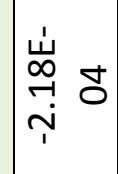 \\
\hline & 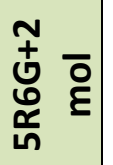 & $\begin{array}{l}\text { शे } \\
\text { g. } \\
0\end{array}$ & $\begin{array}{l}\text { शे } \\
\text { Oे } \\
0\end{array}$ & 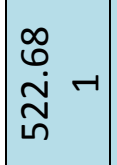 & $\begin{array}{l}\hat{\jmath} \\
\text { - } \\
\text { - }\end{array}$ & סִ & $\mid \begin{array}{ll}0 & \\
\infty & \text { in } \\
\text { in } & \text { n }\end{array}$ & $\begin{array}{l}\ddot{\varphi} \\
\dot{\omega}\end{array}$ & $\begin{array}{l}\hat{A} \\
0 \\
0\end{array}$ & 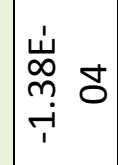 \\
\hline & 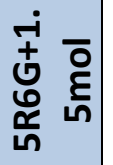 & $\begin{array}{l}\text { হ. } \\
\text { } \\
0\end{array}$ & $\begin{array}{l}\text { शे } \\
\text { Oे } \\
\end{array}$ & $\begin{array}{l}\stackrel{n}{N} \\
\underset{N}{N} \\
\end{array}$ & 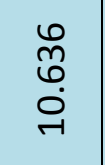 & స్ & 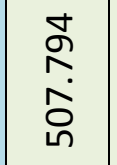 & $\begin{array}{l}\text { ڤ̊ } \\
\stackrel{\text { ஸे }}{N}\end{array}$ & $\begin{array}{l}\vec{A} \\
0 \\
0\end{array}$ & 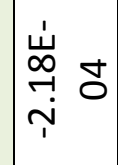 \\
\hline & 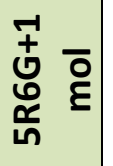 & $\begin{array}{l}\text { gे } \\
\text { } \\
\text {. }\end{array}$ & $\begin{array}{l}\text { शे } \\
\text { Oे } \\
\end{array}$ & $\mid \begin{array}{l}\infty \\
\tilde{N} \\
\tilde{n}\end{array}$ & 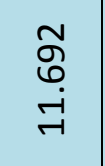 & 合 & \begin{tabular}{ll}
\multirow{N}{*}{} & \\
$\operatorname{Lin}_{n}$ & $\sigma$
\end{tabular} & 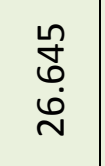 & $\begin{array}{l}\stackrel{D}{\Delta} \\
\stackrel{\Delta}{0} \\
0\end{array}$ & 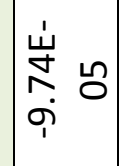 \\
\hline & 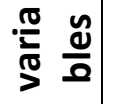 & $\simeq$ & $\begin{array}{l}\overline{\bar{c}} \\
\propto x\end{array}$ & 3 & 요 & $\underset{\sigma}{-1}$ & $\frac{\pi}{3}$ & ฮิ & శี & 이 \\
\hline & 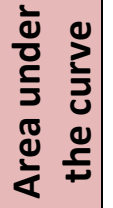 & 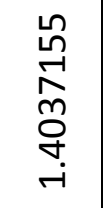 & 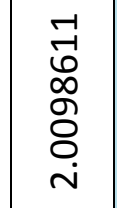 & 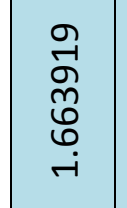 & $\begin{array}{l}-1 \\
\overrightarrow{0} \\
\infty \\
o \\
8 \\
0 \\
i\end{array}$ & 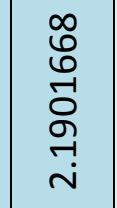 & 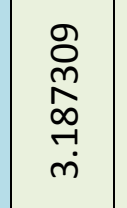 & 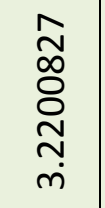 & 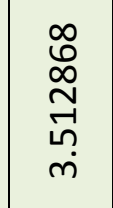 & $\begin{array}{l}\stackrel{+}{m} \\
\stackrel{p}{\infty} \\
\infty \\
0 \\
\dot{+} \\
\dot{+}\end{array}$ \\
\hline & & 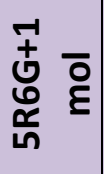 & 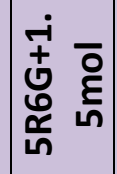 & 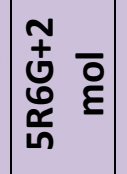 & 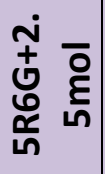 & 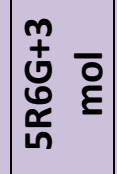 & 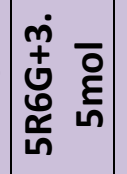 & 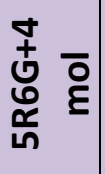 & 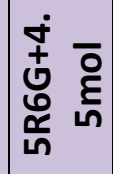 & 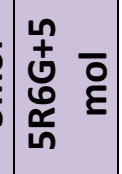 \\
\hline
\end{tabular}




\begin{tabular}{|c|c|c|c|c|c|c|c|c|c|c|}
\hline & 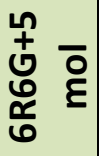 & $\begin{array}{l}\text { शे } \\
\text { Oे }\end{array}$ & $\begin{array}{l}\infty \\
\text { Oे } \\
\text { م. }\end{array}$ & 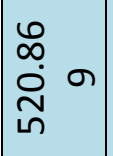 & $\begin{array}{l}\stackrel{\infty}{\infty} \\
\infty \\
\dot{d}\end{array}$ & $\begin{array}{l}\overrightarrow{8} \\
0 \\
0\end{array}$ & $\mid \begin{array}{l}\stackrel{n}{\sim} \\
\stackrel{\sim}{\sim}\end{array}$ & $\begin{array}{l}\text { बे } \\
\text { б̆ }\end{array}$ & $\begin{array}{l}\tilde{N} \\
\stackrel{0}{0} \\
0\end{array}$ & 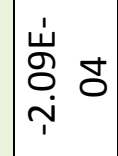 \\
\hline & 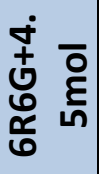 & $\begin{array}{l}\text { જे } \\
\text { જ. } \\
\text { - }\end{array}$ & $\begin{array}{l}\infty \\
\text { Oे } \\
0 \\
0\end{array}$ & 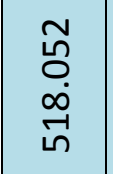 & 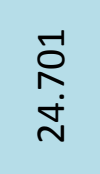 & $\stackrel{n}{0}$ & $\begin{array}{l}\stackrel{-}{n} \\
\stackrel{n}{n} \\
\text { ñ }\end{array}$ & $\begin{array}{l}\text { शे } \\
\text { : } \\
\text { ○े }\end{array}$ & $\begin{array}{l}\text { f } \\
\dot{0}\end{array}$ & 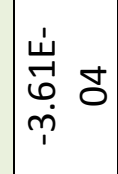 \\
\hline & 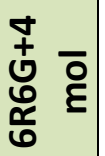 & $\begin{array}{l}\text { হे } \\
\text { Oे } \\
0\end{array}$ & $\begin{array}{l}\text { बे } \\
\text { જ̆ }\end{array}$ & $\begin{array}{l}m \\
\stackrel{\leftrightarrow}{n} \\
\text { ค่ }\end{array}$ & 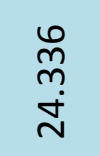 & $\begin{array}{l}8 \\
8 \\
0\end{array}$ & $\mid \begin{array}{ll}\varnothing \\
\ddot{N} \\
\text { N }\end{array}$ & $\begin{array}{l}\hat{0} \\
-1 \\
0 \\
-1\end{array}$ & $\begin{array}{l}\stackrel{2}{y} \\
\text { Oे } \\
0\end{array}$ & 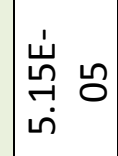 \\
\hline & 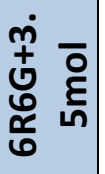 & $\begin{array}{l}\text { gे } \\
\text { Oे. } \\
0\end{array}$ & $\begin{array}{l}\text { शे } \\
\text { Oे } \\
\text { - }\end{array}$ & 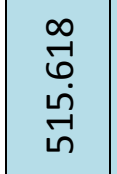 & $\begin{array}{l}\stackrel{\circ}{N} \\
\text { ஸे }\end{array}$ & O্. & 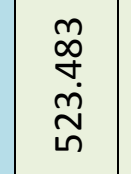 & 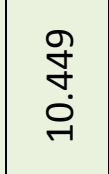 & $\begin{array}{l}0 \\
0 \\
0 \\
0\end{array}$ & 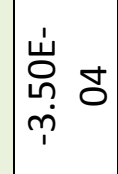 \\
\hline & 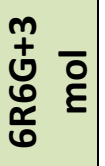 & $\begin{array}{l}\text { शे } \\
\text { Oे } \\
0\end{array}$ & $\begin{array}{l}\text { शे } \\
\text { Oे } \\
0\end{array}$ & $\underset{\text { N̦}}{\stackrel{\infty}{N}}$ & $\begin{array}{l}n \\
\hat{6} \\
0 \\
0 \\
-1\end{array}$ & $\begin{array}{l}\tilde{n} \\
\tilde{O} \\
0\end{array}$ & 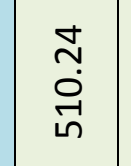 & $\begin{array}{l}\underset{N}{N} \\
\stackrel{\sim}{N}\end{array}$ & $\begin{array}{l}\widehat{̃} \\
0 \\
0 \\
0\end{array}$ & 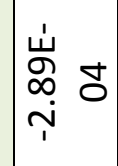 \\
\hline & 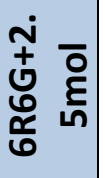 & -1 & $\begin{array}{l}\text { शे } \\
\text { م్ } \\
\text { - }\end{array}$ & $\begin{array}{l}\stackrel{\infty}{N} \\
\underset{N}{N}\end{array}$ & 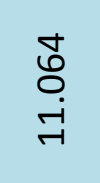 & 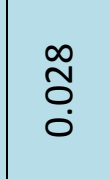 & $\begin{array}{l}\stackrel{0}{m} \\
m \\
\ddot{\theta} \\
\text { மn }\end{array}$ & 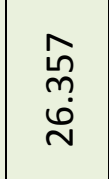 & 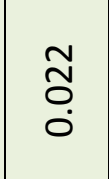 & 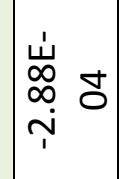 \\
\hline & 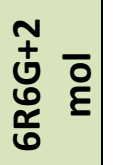 & $\begin{array}{l}\text { शे } \\
\text { مे }\end{array}$ & $\begin{array}{l}\text { शे } \\
\text { Oे }\end{array}$ & $\underset{\stackrel{m}{\sim}}{\stackrel{m}{\sim}} \wedge$ & $\begin{array}{l}\overrightarrow{0} \\
0 \\
- \\
\rightarrow \\
-\end{array}$ & $\begin{array}{l}\stackrel{\Xi}{\sim} \\
\stackrel{0}{0}\end{array}$ & 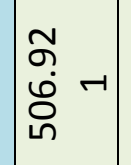 & $\begin{array}{l}\dot{b} \\
0 \\
\dot{\omega} \\
\text { N }\end{array}$ & סִ & 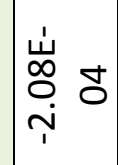 \\
\hline & 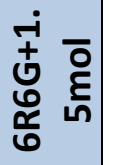 & $\begin{array}{l}\text { হ. } \\
\text { } \\
0\end{array}$ & $\begin{array}{l}\text { ò } \\
\text { Oे } \\
0\end{array}$ & 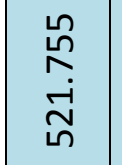 & $\begin{array}{l}\text { Õ } \\
\text { ษे } \\
\text { ஸे }\end{array}$ & $\begin{array}{l}\mathscr{0} \\
\tilde{O} \\
0\end{array}$ & $\begin{array}{l}\text { N } \\
\text { fं }\end{array}$ & $\begin{array}{l}\underset{\infty}{\infty} \\
m \\
\infty \\
m \\
m\end{array}$ & 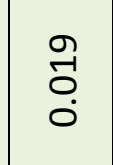 & $\begin{array}{l}-1 \\
8 \\
0 \\
\end{array}$ \\
\hline & 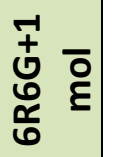 & $\begin{array}{l}\text { g. } \\
\text { } \\
0\end{array}$ & $\begin{array}{l}\text { शे } \\
\text { O़ }\end{array}$ & $\left|\begin{array}{l}\varnothing \\
\infty \\
\infty \\
n \\
n\end{array}\right|$ & 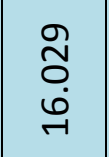 & $\begin{array}{l}\stackrel{+}{-} \\
\stackrel{0}{0}\end{array}$ & $\mid \begin{array}{l}\stackrel{n}{N} \\
\dot{\varphi} \\
\stackrel{\circ}{n}\end{array}$ & $\begin{array}{l}m \\
\stackrel{n}{0} \\
\infty \\
\text { N }\end{array}$ & $\begin{array}{l}\hat{y} \\
\text { ó } \\
0\end{array}$ & 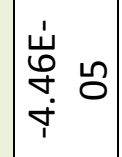 \\
\hline & 永 & $\simeq$ & $\begin{array}{l}\overline{\bar{u}} \\
\check{\check{c}}\end{array}$ & 3 & تح & స్ర & $\tilde{3}$ & ฮ & ช & ? \\
\hline & 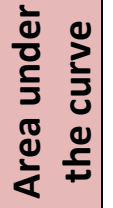 & 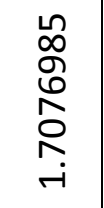 & 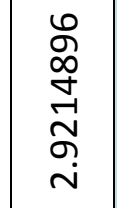 & 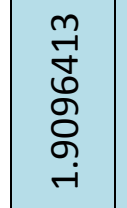 & 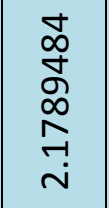 & 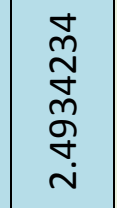 & \begin{tabular}{l}
$\stackrel{m}{N}$ \\
\multirow{N}{N}{} \\
$\stackrel{N}{N}$ \\
$m$ \\
$m$
\end{tabular} & 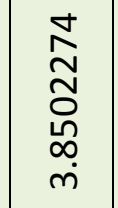 & $\begin{array}{l}\infty \\
\stackrel{\infty}{\$} \\
\hat{\sigma} \\
\stackrel{+}{+}\end{array}$ & 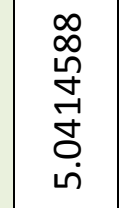 \\
\hline & & 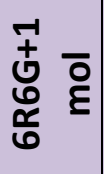 & 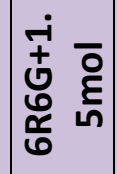 & 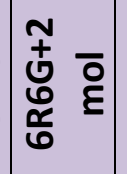 & 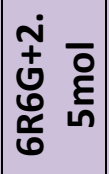 & 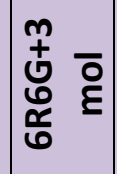 & 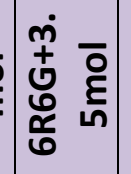 & 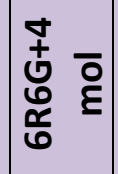 & 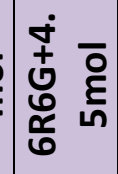 & 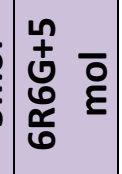 \\
\hline
\end{tabular}




\begin{tabular}{|c|c|c|c|c|c|c|c|c|c|c|}
\hline & 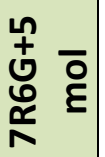 & $\begin{array}{l}\text { Oे } \\
\text { Oे }\end{array}$ & $\begin{array}{l}\infty \\
\text { ô. } \\
\text { م. }\end{array}$ & $\begin{array}{l}\hat{N} \\
0 \\
0 \\
\text { N }\end{array}$ & $\begin{array}{l}\text { Ñ } \\
\infty \\
\dot{J}\end{array}$ & 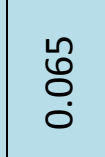 & 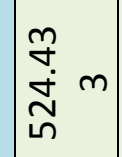 & $\begin{array}{l}-1 \\
0 \\
0 \\
0 \\
0\end{array}$ & $\begin{array}{l}\text { L } \\
\text { L } \\
0 \\
0\end{array}$ & 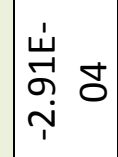 \\
\hline & 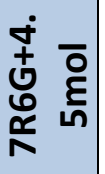 & $\begin{array}{l}\text { જे } \\
\text { જ. } \\
\text { - }\end{array}$ & $\begin{array}{l}\text { बे } \\
\text { م̆ }\end{array}$ & $\begin{array}{l}\hat{n} \\
\stackrel{0}{0} \\
\infty \\
\end{array}$ & 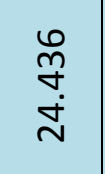 & $\begin{array}{l}\hat{n} \\
\text { ڤ̊ }\end{array}$ & $\begin{array}{l}\infty \\
\infty \\
0 \\
\stackrel{\Delta}{+} \\
\text { ก }\end{array}$ & $\begin{array}{l}\hat{8} \\
0 \\
0\end{array}$ & $\begin{array}{l}\text { 랭 } \\
\text { Oํ. }\end{array}$ & 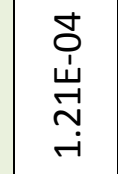 \\
\hline & 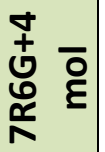 & $\begin{array}{l}\text { হे } \\
\text { Oे } \\
0\end{array}$ & $\begin{array}{l}\infty \\
\text { ò } \\
\text { o. }\end{array}$ & $\begin{array}{l}-1 \\
0 \\
\dot{\vec{j}}\end{array}$ & $\begin{array}{l}\stackrel{J}{N} \\
\stackrel{\Delta}{\sim}\end{array}$ & $\begin{array}{l}\stackrel{g}{0} \\
\text { Oें }\end{array}$ & $\begin{array}{l}\stackrel{0}{\sim} \\
\stackrel{\sim}{N} \\
\text { ñ. }\end{array}$ & 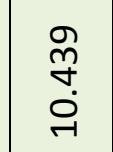 & $\begin{array}{l}\text { 로 } \\
\text { م. }\end{array}$ & 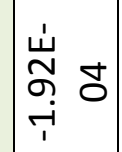 \\
\hline & 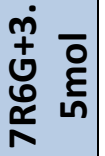 & $\begin{array}{l}\text { ने } \\
\text { Оे }\end{array}$ & $\begin{array}{l}\text { शे } \\
\text { Oे } \\
0\end{array}$ & 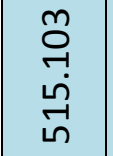 & 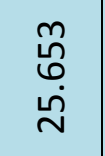 & $\begin{array}{l}\mathcal{Z} \\
\stackrel{0}{0}\end{array}$ & 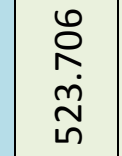 & $\begin{array}{l}\stackrel{+}{n} \\
\text { m. } \\
0 \\
\sim\end{array}$ & 年 & 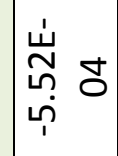 \\
\hline & 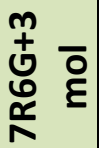 & $\begin{array}{l}\text { शे } \\
\text { के }\end{array}$ & $\begin{array}{l}\text { शे } \\
\text { م̆ } \\
\text {. }\end{array}$ & $\mid \begin{array}{l}0 \\
\mathbb{C} \\
\mathbb{N} \\
\text { ñ }\end{array}$ & $\begin{array}{l}\infty \\
\infty \\
6 \\
0 \\
0 \\
-1\end{array}$ & $\begin{array}{l}\mathscr{0} \\
\ddot{0} \\
0\end{array}$ & $\begin{array}{l}\Omega \\
\hat{\sigma} \\
\delta \\
\dot{D}\end{array}$ & $\begin{array}{l}\text { ñ } \\
\stackrel{+}{d} \\
\stackrel{n}{N}\end{array}$ & $\begin{array}{l}\text { Оิ } \\
0 \\
0\end{array}$ & 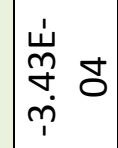 \\
\hline & 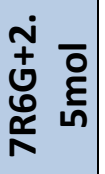 & $\begin{array}{l}\text { g. } \\
\text {. } \\
0\end{array}$ & $\begin{array}{l}\text { शे } \\
\text { Oे } \\
0\end{array}$ & 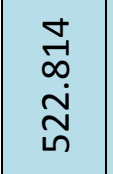 & 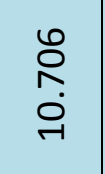 & $\begin{array}{l}\mathscr{\infty} \\
\tilde{O} \\
0 \\
0\end{array}$ & 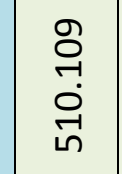 & 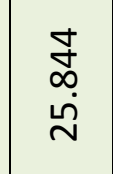 & $\begin{array}{l}\text { న్ } \\
\text { Oे. } \\
0\end{array}$ & 岗 \\
\hline & 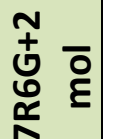 & $\begin{array}{l}\text { S. } \\
\text { } \\
0\end{array}$ & $\begin{array}{l}\text { જे } \\
\text { م. } \\
\text { - }\end{array}$ & $\begin{array}{l}\tilde{N} \\
\underset{N}{N} \\
\end{array}$ & 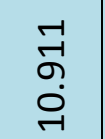 & $\begin{array}{l}\stackrel{\infty}{0} \\
0 \\
0\end{array}$ & $\left.\mid \begin{array}{l}\infty \\
0 \\
\dot{0} \\
0 \\
i\end{array}\right)$ & 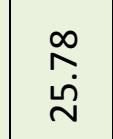 & $\begin{array}{l}\widetilde{\Xi} \\
0 \\
0\end{array}$ & 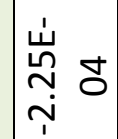 \\
\hline & 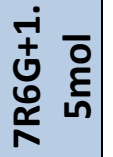 & $\begin{array}{l}\text { g. } \\
\text {. } \\
0\end{array}$ & $\begin{array}{l}\text { ò } \\
\text { Oे } \\
0\end{array}$ & 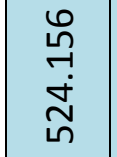 & $\underset{\stackrel{\sim}{+}}{\stackrel{\sim}{\sim}}$ & $\begin{array}{l}\check{Z} \\
0 \\
0\end{array}$ & 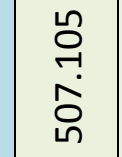 & $\begin{array}{l}0 \\
\tilde{n} \\
\stackrel{n}{N}\end{array}$ & $\begin{array}{l}\infty \\
\stackrel{0}{0} \\
0\end{array}$ & 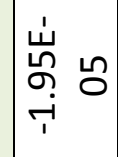 \\
\hline & 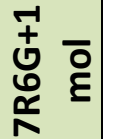 & $\begin{array}{l}8 \\
\qquad \\
0 \\
0\end{array}$ & $\begin{array}{l}\infty \\
\text { Oे } \\
0 \\
0\end{array}$ & 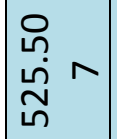 & 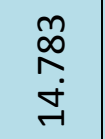 & $\tilde{O}$ & $\begin{array}{l}0 \\
m \\
\text { m. } \\
\text { மn }\end{array}$ & $\begin{array}{l}\infty \\
\stackrel{\infty}{-1} \\
\infty \\
\stackrel{\infty}{\sim}\end{array}$ & $\begin{array}{l}\text { ñ } \\
\text { o. } \\
0\end{array}$ & $\begin{array}{l}\stackrel{\leftrightarrow}{\sim} \\
\text { ஸn }\end{array}$ \\
\hline & $\frac{\pi}{\frac{\pi}{5}} \frac{\boldsymbol{y}}{0}$ & $\simeq$ & $\begin{array}{l}\grave{\bar{v}} \\
\stackrel{w}{\simeq}\end{array}$ & 3 & ق & $\vec{\sigma}$ & $\tilde{3}$ & กิ & $\widetilde{\pi}$ & $\stackrel{1}{1}$ \\
\hline & 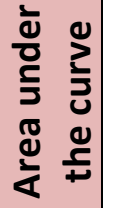 & $\begin{array}{l}\hat{n} \\
\infty \\
\hat{\Upsilon}^{\circ} \\
\infty \\
-i\end{array}$ & 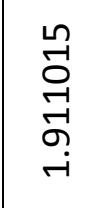 & 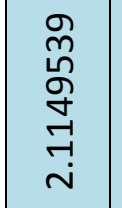 & 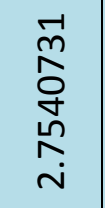 & 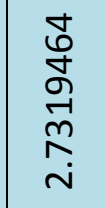 & $\begin{array}{l}\vec{n} \\
\hat{0} \\
0 \\
0 \\
\hat{n} \\
\dot{m}\end{array}$ & 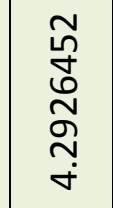 & $\begin{array}{l}\infty \\
\stackrel{\infty}{\infty} \\
\infty \\
\stackrel{-}{\sigma} \\
\stackrel{1}{\delta} \\
\dot{\sigma}\end{array}$ & 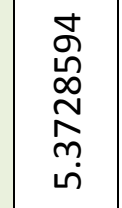 \\
\hline & & 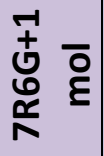 & 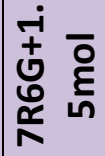 & 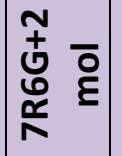 & 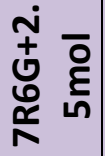 & 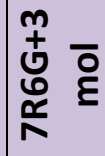 & 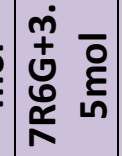 & 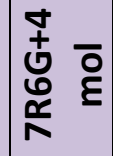 & 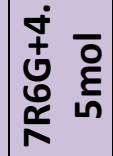 & 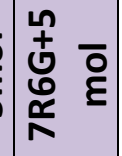 \\
\hline
\end{tabular}




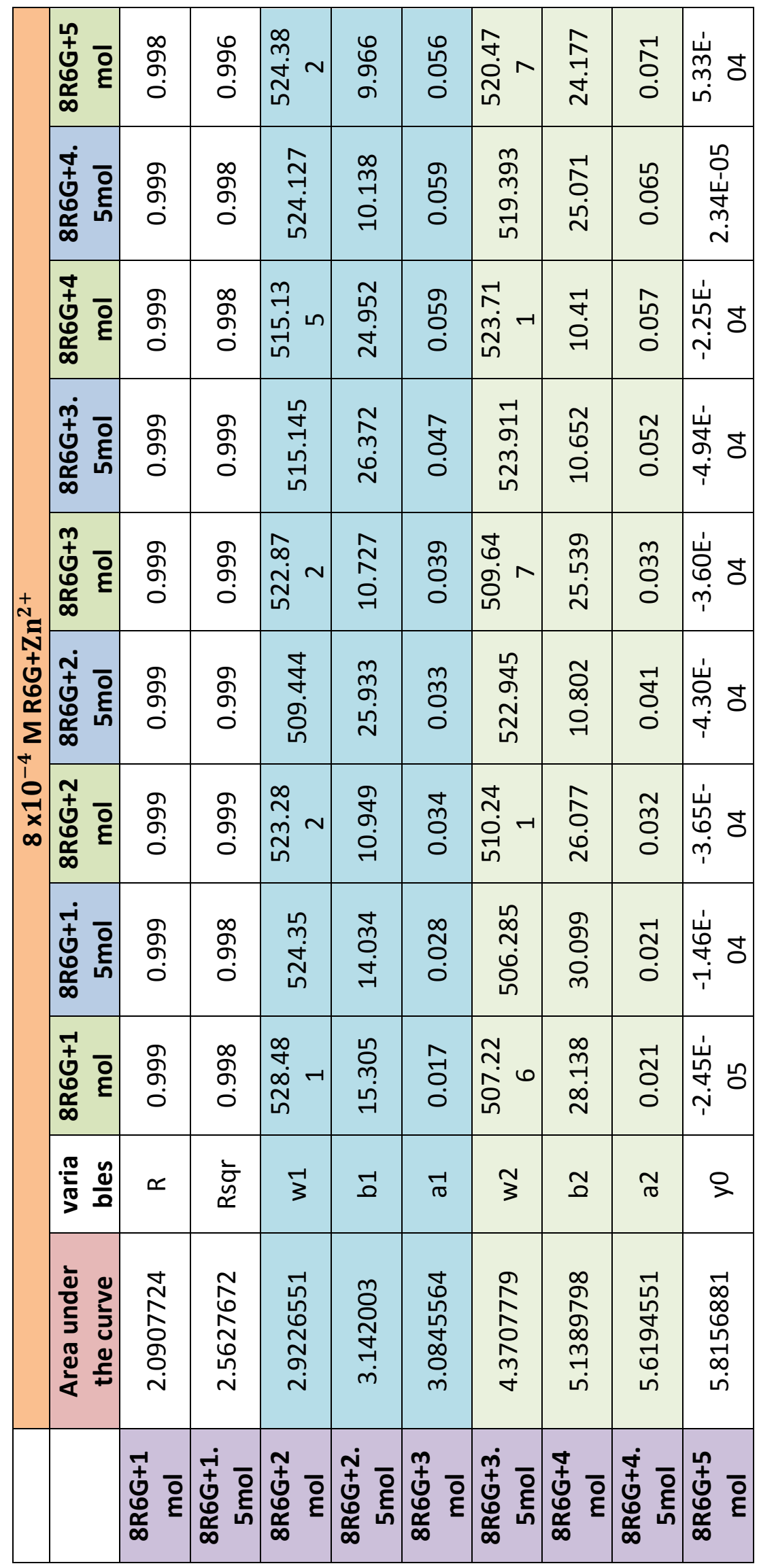




\begin{tabular}{|c|c|c|c|c|c|c|c|c|c|c|}
\hline & 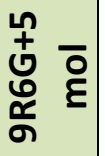 & $\begin{array}{l}\text { Oे } \\
\text { Oे } \\
\text { - }\end{array}$ & $\begin{array}{l}\infty \\
\text { Oे } \\
\text { م. }\end{array}$ & $\begin{array}{l}\tilde{N} \\
\underset{\sim}{N}\end{array}$ & 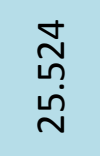 & $\begin{array}{l}\stackrel{1}{0} \\
\infty \\
0 \\
0\end{array}$ & 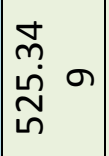 & $\begin{array}{l}\text { N } \\
\text { ñ } \\
0 \\
\text { - }\end{array}$ & $\begin{array}{l}\mathbb{N} \\
0 \\
0\end{array}$ & 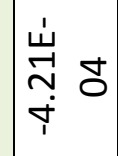 \\
\hline & 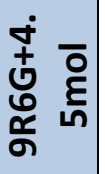 & $\begin{array}{l}\text { જे } \\
\text { જ. } \\
\text { - }\end{array}$ & $\begin{array}{l}\infty \\
\text { Oे } \\
0 \\
0\end{array}$ & 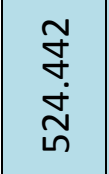 & $\begin{array}{l}\text { స̃ } \\
\stackrel{9}{9}\end{array}$ & $\begin{array}{l}\qquad 0 \\
0 \\
0 \\
0\end{array}$ & 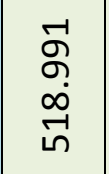 & 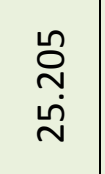 & $\begin{array}{l}\hat{a} \\
0 \\
0\end{array}$ & 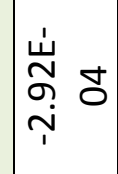 \\
\hline & 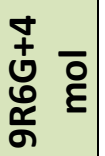 & $\begin{array}{l}\text { হे } \\
\text { Oे } \\
0\end{array}$ & $\begin{array}{l}\text { के } \\
\text { ŏ }\end{array}$ & 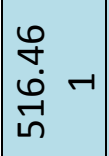 & 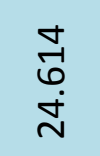 & $\begin{array}{l}-1 \\
0 \\
0\end{array}$ & 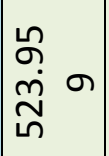 & $\begin{array}{l}\tilde{N} \\
\stackrel{\rho}{\rho}\end{array}$ & $\begin{array}{l}\text { 足 } \\
\text { O̊. } \\
0\end{array}$ & 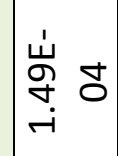 \\
\hline & 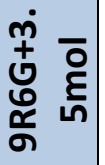 & $\begin{array}{l}\text { के } \\
\text { o. } \\
\text { - }\end{array}$ & $\begin{array}{l}\hat{0} \\
0 \\
0 \\
0\end{array}$ & 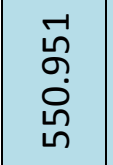 & $\begin{array}{l}8 \\
0 \\
0 \\
\dot{y} \\
\rightarrow\end{array}$ & $\begin{array}{l}0 \\
0 \\
\vdots \\
1\end{array}$ & 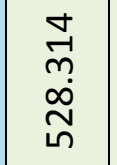 & $\begin{array}{l}\mathscr{D} \\
\ddot{0} \\
\ddot{N}\end{array}$ & 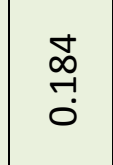 & $\begin{array}{l}-1 \\
0 \\
0\end{array}$ \\
\hline & 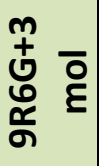 & $\begin{array}{l}\text { g. } \\
\text {. } \\
0\end{array}$ & $\begin{array}{l}\text { જे } \\
\text { م. } \\
\text { - }\end{array}$ & $\begin{array}{l}N \\
\hat{O} \\
-1 \\
\text { in }\end{array}$ & $\begin{array}{l}\text { :े } \\
\text { ดे } \\
\text { ஸे }\end{array}$ & $\begin{array}{l}\vec{y} \\
\dot{0}\end{array}$ & $\begin{array}{l}\stackrel{n}{2} \\
\underset{N}{N}\end{array}$ & $\begin{array}{l}-1 \\
\infty \\
0 \\
0 \\
-1\end{array}$ & \begin{tabular}{l}
\multirow{Z}{*}{} \\
$\dot{0}$
\end{tabular} & 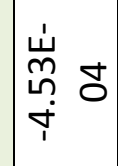 \\
\hline & 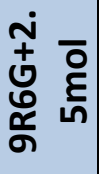 & $\begin{array}{l}\text { } \\
\text { مे } \\
0\end{array}$ & $\begin{array}{l}\text { शे } \\
\text { Oे } \\
0\end{array}$ & 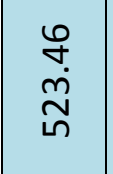 & 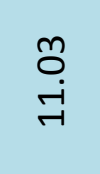 & $\begin{array}{l}0 \\
\tilde{0} \\
0 \\
0\end{array}$ & $\begin{array}{l}\hat{m} \\
\text { ò } \\
\text { in }\end{array}$ & $\begin{array}{l}\hat{-} \\
\dot{0} \\
\dot{\sim}\end{array}$ & $\begin{array}{l}\stackrel{\nabla}{0} \\
0 \\
0\end{array}$ & 岁 \\
\hline P & 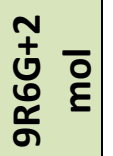 & $\begin{array}{l}\text { शे } \\
\text { م़े }\end{array}$ & $\begin{array}{l}\text { शे } \\
\text { Oे }\end{array}$ & $\begin{array}{l}\text { o } \\
\text { N்} \\
\text { ñ }\end{array}$ & $\begin{array}{l}m \\
0 \\
\dot{-} \\
\overrightarrow{-}\end{array}$ & $\begin{array}{l}\mathscr{ల} \\
0 \\
0 \\
0\end{array}$ & $\begin{array}{l}\text { mे } \\
\text { òं } \\
\text { in }\end{array}$ & $\begin{array}{l}\text { ने } \\
\text { ọ } \\
\dot{\emptyset}\end{array}$ & $\begin{array}{l}\tilde{m} \\
0 \\
0 \\
0\end{array}$ & 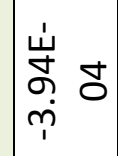 \\
\hline & 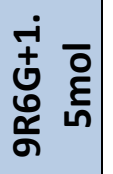 & $\begin{array}{l}\text { g. } \\
\text {. } \\
0\end{array}$ & $\begin{array}{l}\infty \\
\text { } \\
0 \\
0\end{array}$ & $\begin{array}{l}\underset{N}{ } \\
\infty \\
\dot{\sim} \\
\text { N }\end{array}$ & 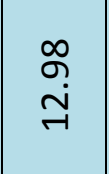 & స్ & $\begin{array}{l}\stackrel{+}{N} \\
\text { ம் } \\
\text { ํํ }\end{array}$ & $\begin{array}{l}m \\
\infty \\
\sim \\
\stackrel{1}{m} \\
m\end{array}$ & 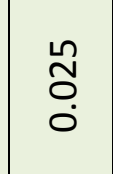 & $\begin{array}{l}\stackrel{2}{0} \\
\dot{1} \\
\stackrel{\sigma}{\widehat{r}} \\
\dot{m}\end{array}$ \\
\hline & 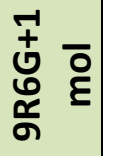 & $\begin{array}{l}\text { gे } \\
\text { } \\
\text {. }\end{array}$ & $\begin{array}{l}\text { ò } \\
\text { Oे } \\
0\end{array}$ & $\mid \begin{array}{ll}\tilde{n} & \\
\infty & m \\
\sim & m\end{array}$ & $\begin{array}{l}\infty \\
\stackrel{1}{0} \\
\infty \\
\infty \\
\sim\end{array}$ & $\underset{\widetilde{O}}{\tilde{O}}$ & $\mid \begin{array}{l}\infty \\
\infty \\
\stackrel{2}{\infty} \\
\stackrel{\sigma}{\sigma}\end{array}$ & $\begin{array}{l}\mathscr{Z} \\
\text { ঠें }\end{array}$ & $\begin{array}{l}\tilde{O} \\
\tilde{O}\end{array}$ & 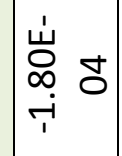 \\
\hline & 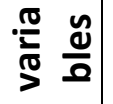 & $\simeq$ & $\begin{array}{l}\overline{\bar{c}} \\
\propto x\end{array}$ & 3 & 요 & $\underset{\sigma}{-1}$ & $\frac{\pi}{3}$ & ฮิ & ָ & ? \\
\hline & 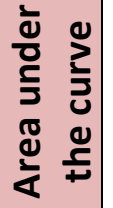 & 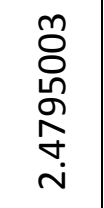 & 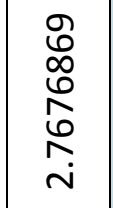 & 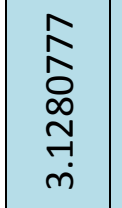 & 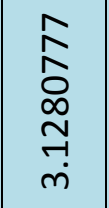 & $\begin{array}{l}\underset{D}{d} \\
\infty \\
0 \\
\stackrel{p}{n} \\
\stackrel{n}{n} \\
m\end{array}$ & 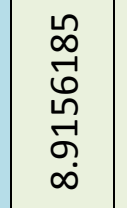 & 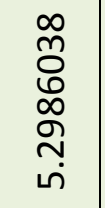 & $\begin{array}{l}\overrightarrow{1} \\
\hat{0} \\
\tilde{N} \\
\stackrel{0}{0} \\
\dot{\varphi}\end{array}$ & 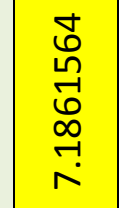 \\
\hline & & 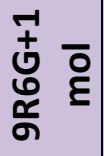 & 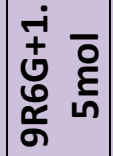 & 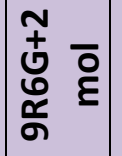 & 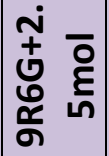 & 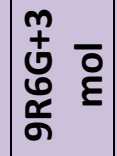 & 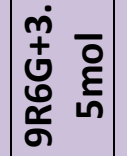 & 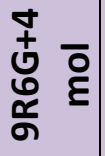 & 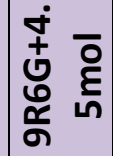 & 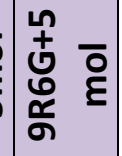 \\
\hline
\end{tabular}




\section{REFERENCES}

1. Yang, J.-S. and Swager, T. M. Fluorescent Porous Polymer Films as TNT Chemosensors: Electronic and Structural Effects. J. Am. Chem. Soc. 1998, 120, $11864-11873$.

2. Sunahara, H.; Urano, Y.; Kojima, H; and Nagano, T., Design and Synthesis of a Library of BODIPY-Based Environmental Polarity Sensors Utilizing Photoinduced Electron-Transfer-Controlled Fluorescence ON/OFF Switching. J. Am. Chem. Soc. 2007, 129, 5597 - 5604.

3. Levitsky, I. A., Euler, W. B., Tokranova, N., and Rose, A., Fluorescent Polymer - Porous Silicon Microcavity Devices for Explosives Detection. Appl. Phys. Lett. 2007, 90, 041904-1 - 041904-3.

4. Satrijo, A.; Kool, S. E.; and Swager, T. M., Enhanced Luminescence from Emissive Defects in Aggregated Conjugated Polymers. Macromolecules 2007, $40,8833-8841$.

5. Qin, A.; Lam, J. W. Y.; Tang, L.; Jim, C. K. W.; Zhao, H.; Sun, J.; and Tang, B. Z., Polytriazoles with Aggregation-Induced Emission Characteristics: Synthesis by Click Polymerization and Application as Explosive Chemosensors. Macromolecules 2009, 42, 1421 - 1424.

6. Yuan, W. Z.; Zhao, H.; Shen, X. Y.; Mahtab, F.; Lam, J. W. Y.; Sun, J. Z.; and Tang, B. Z., Luminogenic Polyacetylenes and Conjugated Polyelectrolytes: Synthesis, Hybridization with Carbon Nanotubes, Aggregation-Induced Emission, Superamplification in Emission Quenching by Explosives, and Fluorescent Assay for Protein Quantitation. Macromolecules 2009, 42, 9400 9411.

7. $\quad$ Liu, J.; Zhong, Y.; Lam, J. W. Y.; Lu, P.; Hong, Y.; Yu, Y.; Yue, Y.; Faisal, M.; Sung, H. H. Y.; Williams, I. D.; Wong, K. S.; and Tang, B. Z., Hyperbranched Conjugated Polysiloles: Synthesis, Structure, Aggregation-Enhanced Emission, Multicolor Fluorescent Photopatterning, and Superamplified Detection of Explosives. Macromolecules 2010, 43, 4921 - 4936. 
8. Zhang, Z.; Zheng, Y.; Hang, W.; Yan, X.; and Zhao, Y., Sensitive and Selective Off-On Rhodamine Hydrazide Fluorescent Chemosensor for Hypochlorous Acid Detection and Bioimaging. Talanta 2011, 85, 779 - 786.

9. Freeman, R.; Finder, T.; Bahshi, L.; Gill, R.; and Willner, I., Functionalized CdSe/ZnS QDs for the Detection of Nitroaromatic or RDX Explosives. $A d v$. Mater. 2012, 24, 6416 - 6421.

10. Latendresse, C. A.; Fernandes, S. C.; You, S.; Zhang, H. Q.; and Euler, W. B., A Fluorometric Sensing Array for the Detection of Military Explosives and IED Materials. Anal. Methods 2013, 5, 5457 - 5463.

11. Matoian, M. A.; Sweetman, R.; Hall, E. C.; Albanese, S.; and Euler, W. B., Light Trapping to Amplify Metal Enhanced Fluorescence with Application for Sensing TNT. J. Fluoresc. 2013, 23, $877-880$.

12. de Greñu, B. D.; Moreno, D.; Torroba, T.; Berg, A.; Gunnars, J.; Nilsson, T.; Nyman, R.; Persson, M.; Pettersson, J.; Eklind, I.; and Wästerby, P., Fluorescent Discrimination between Traces of Chemical Warfare Agents and Their Mimics. J. Am. Chem. Soc. 2014, 136, 4125 - 4128.

13. Xu, W., Ren, C., Teoh, C. L., Peng, J., Gadre, S. H., Rhee, H.-W., Lee, C.-L. K., and Chang, Y.-T., An Artificial Tongue Fluorescent Sensor Array for Identification and Quantitation of Various Heavy Metal Ions. Anal. Chem. 2014, $86,8763-8769$.

14. Wang, S.; Ding, L.; Fan, J.; Wang, Z.; and Fang, Y., Bispyrene/SurfactantAssembly-Based Fluorescent Sensor Array for Discriminating Lanthanide Ions in Aqueous Solution. ACS Appl. Mater. Interfaces 2014, 6, 16156 - 16165.

15. Zhang, H. Q. and Euler, W. B., Detection of Gas-Phase Explosive Analytes Using Fluorescent Spectroscopy of Thin Films of Xanthene Dyes. Sens. Actuators B 2016, 225, $553-562$.

16. Staahlberg, J. and Almgren, M., Polarity of Chemically Modified Silica Surfaces and Its Dependence on Mobile-Phase Composition by Fluorescence Spectrometry. Anal. Chem. 1985, 57, $817-821$. 
17. Sackett, D.L. and Wolff, J., Nile Red as a Polarity-Sensitive Fluorescent Probe of Hydrophobic Protein Surfaces. Anal Biochem. 1987, 167, 228 - 234.

18. Chang, K. and Forcé, R. K., Time-Resolved Pyrene Fluorescence for Determination of Polymer Surface Polarity: Correlations with Surface Tension. Appl. Spectr. 1995, 49, $211-215$.

19. Hayashi, Y.; Kawada, Y.; and Ichimura, K., Dicyanoanthracene as a Fluorescence Probe for Studies on Silica Surfaces. Langmuir 1995, 11, 2077 2082.

20. Baldi, L. D. C.; Iamazaki, E. T.; and Atvars, T. D. Z., Evaluation of the Polarity of Polyamide Surfaces Using the Fluorescence Emission of Pyrene. Dyes and Pigments 2008, 76, $669-676$.

21. Habenicht, S. H.; Schramm, S.; Fischer, S.; Sachse, T.; Herrmann-Westendorf, F.; Bellmann, A.; Dietzek, B.; Presselt, M.; Weiß, D.; Beckert, R.; and Görlse, H., Tuning the Polarity and Surface Activity of Hydroxythiazoles - Extending the Applicability of Highly Fluorescent Self-Assembling Chromophores to Supra-Molecular Photonic Structures. J. Mater. Chem. C 2016, 4, 958 - 971.

22. McCrate, J. M. and Ekerdt, J. G., Coverage-Dependent Luminescence from Two-Dimensional Systems of Covalently Attached Perylene Fluorophores on Silica. J. Phys. Chem. C 2014, 118, $2104-2114$.

23. Arbeloa, F. L.; Gonzalez, I. L.; Ojeda, P. R.; and Arbeloa, I. L., Aggregate Formation of Rhodamine 6G in Aqueous Solution. J. Chem. Soc. Faraday Trans. 2 1982, 78, 989 - 994.

24. Valdes-Aguilera, O. and Neckers, D. C., Aggregation Phenomena in Xanthene Dyes. Acc. Chem. Res. 1989, 22, $171-177$.

25. Ojeda, P. R.; Amashta, I. A. K.; Ochoa, J. R.; and Arbeloa, I. L., Excitonic Treatment and Bonding of Aggregates of Rhodamine 6G in Ethanol. J. Chem. Soc. Faraday Trans. 2 1988, 84, $1-8$.

26. Kemnitz, K. and Yoshihara, K., Entropy-Driven Dimerization of Xanthene Dyes in Nonpolar Solution and Temperature-Dependent Fluorescence Decay 
of Dimers. J. Phys. Chem. 1991, 95, $6095-6104$.

27. Elking, M. D.; He, G.; and Xu, Z., Molecular Orientation of Submonolayer Rhodamine-6G on Quartz Substrates: A Comparative Study Using Reflection and Transmission UV-Vis Spectroscopy. J. Chem. Phys. 1996, 105, 6565 6573.

28. Sasai, R.; Fujita, T.; Iyi, N.; Itoh, H.; and Takagi, K., Aggregated Structures of Rhodamine 6G Intercalated in a Fluor-Taeniolite Thin Film. Langmuir 2002, $18,6578-6583$.

29. Magde, D.; Wong, R.; Seybold, P. G. Fluorescence Quantum Yields and Their Relation to Lifetimes of Rhodamine $6 \mathrm{G}$ and Fluorescein in Nine Solvents: Improved Absolute Standards for Quantum Yields Photochem. Photobiol. 2002, 75, 327-334.

30. Martinez, V. M.; Arbeloa, F. L.; Prieto, J. B., Lopez, T. A.; and Arbeloa, I. L., Characterization of Rhodamine 6G Aggregates Intercalated in Solid Thin Films of Laponite Clay. 1. Absorption Spectroscopy. J Phys. Chem. B 2004, $108,20030-20037$.

31. Fedoseeva, M.; Letrun, R.; Vauthey, E. Excited-State Dynamics of Rhodamine 6G in Aqueous Solution and at the Dodecane/Water Interface. J. Phys. Chem. B 2014, 118, 5184-5193.

32. Chapman, M.; Mullen, M.; Novoa-Ortega, E.; Alhasani, M.; Elman, J. F.; Euler, W. B. Structural Evolution of Ultrathin Films of Rhodamine 6G on Glass. J. Phys. Chem. C 2016, 120, $8289-8297$.

33. Mullen, M; and Euler, W. B., The Influence of Interfacial Effects of the Electronic Properties of Rhodamine 6G on PVDF. Langmuir 2017, 33, 2194 2204.

34. Prest, W. M.; and Luca, D. J., The Morphology and Thermal Response of High-Temperature-Crystallized Poly(vinylidene fluoride). J. Appl. Phys. 1975, 46, $4136-4143$. 
35. Lovinger, A. J.; and Keith, H. D., Electron Diffraction Investigation of a HighTemperature Form of Poly(vinylidene fluoride). Macromolecules 1979, 12, $919-924$.

36. Benz, M.; Euler, W. B.; Gregory, O. J. The Influence of Preparation Conditions on the Surface Morphology of Poly(vinylidene fluoride) Films. Langmuir 2001, 17, 239-243.

37. Benz, M.; Euler, W. B.; Gregory, O. J. The Role of Solution Phase Water on the Deposition of Thin Films of Poly(vinylidene fluoride). Macromolecules 2002, 35, $2682-2688$.

38. Benz, M.; Euler, W. B. Determination of the Crystalline Phases of Poly(vinylidene fluoride) Under Different Preparation Conditions using Differential Scanning Calorimetry and Infrared Spectroscopy. J. Appl. Polym. Sci. 2003, 89, $1093-1100$.

39. Branciforti, M. C.; Sencadas, V.; Lanceros-Mendez, S.; Gregorio Jr, R. New Technique of Processing Highly Oriented Poly(vinylidene fluoride) Films Exclusively in the $\beta$ Phase. J. Polymer Sci. Part B 2007, 45, 2793 - 2801.

40. Yingxue, X.; Huiqing, F.; Weiguo, L.; Chen, Y.; Xiaoling, N.A Study on Thermo-optic Effect of $\beta$ Poly(vinylidenefluoride) Thin Films Prepared by Solution Casting Method. Surf. Rev. Lett. 2008, 15, 175 - 181.

41. Martins, P.; Nunes, J. S.; Hungerford, G.; Miranda, D.; Ferreira, A.; Sencadas, V.; Lanceros-Mendez, S. LocalVariation of the Dielectric Properties of Poly(vinylidene fluoride) During the $\alpha$ - to $\beta$-Phase Transformation. Phys. Lett. A 2009, 373, 177 - 180 .

42. Sencadas, V.; Lanceros-Mendez, S.; Gregorio Jr, R. $\alpha$ to $\beta$ Phase Transformation and Microstructural Changes of PVDF Films Induced by Uniaxial Stretch. J. Macromol. Sci. B: Phys. 2009, 48, $514-525$.

43. Vijayakumar, R. P.; Khakhar, D. V.; Misra, A. Studies on $\alpha$ to $\beta$ Phase Transformations in Mechanically Deformed PVDF Films. J. Appl. Polym. Sci. 2010, 117, $3491-3497$. 
44. Fontaine, K.; Lopez, W.; Crisman, E.; Derov, J.; Euler, W. B., Doping of Polyvinylidene Difluoride with Cobalt Nitrate: Structural, Electrical, and Magnetic Properties. J. Polym. Sci. A: Polym. Chem. 2012, 50, 3970 - 3975. 


\section{CHAPTER 2}

The Fluorescent Spectroscopy of $\mathrm{Zn}^{2+}$-Doped PMMA of Rhodamine 6G

$$
\text { Mona Alhasani, }{ }^{1} \text { William B. Euler }{ }^{1 *}
$$

1. Department of Chemistry, University of Rhode Island, 140 Flagg Road, Kingston, RI 02881

* Corresponding Authors weuler@chm.uri.edu, phone 401-874-5090, fax 401-8745072 .

The following is submitted to the Journal of Langmuir (ACS), and is presented here in manuscript format. 


\section{ABSTRACT}

An investigation of the role of the polymer substrate was attempted, the solvent and the reaction between $\mathrm{Rh} 6 \mathrm{G}$ and $\mathrm{Zn}^{2+}$. Studying the reaction between $\mathrm{Rh} 6 \mathrm{G}$ and $\mathrm{Zn}^{2+}$ was done on glass slides in the absence of polymer. In order to study the role of the solvent, films of PVDF doped with $\mathrm{Zn}^{2+}$ were cast from pure acetone. PVDF polymer was replaced by PMMA to evaluate the role of the polymer in the films. 


\section{INTRODUCTION}

Fluorescence spectroscopy is a very powerful tool that has been used in many areas, such as analytical chemistry, biology and sensors. Detecting explosives is one of the most challenging applications for fluorescence sensing ${ }^{1-2}$, which has been an interest for several years. ${ }^{3,4-5}$ In this work, Rhodamine $6 \mathrm{G}$ is used as the fluorophore because it exhibits some interesting chemistry. Rhodamine 6G has well known optical properties. However, when working with solid phase dyes, the geometry of the layered molecules becomes important. The geometry of neighboring molecules can lead to aggregation, which affects the quantum yields of fluorescent dyes. The spectroscopic properties can be affected by the type and the degree of the aggregation (dimer, trimer, etc.). Exciton theory ${ }^{6,7-8}$ can explain these absorptions. Commonly, three types of dimers (H- non-emissive), ( $\mathrm{J}$ - emissive) and an intermediate oblique orientation are identified by the angle between the monomer dipoles. The H-dimer has a face-to-face arrangement $\left(\theta=0^{\circ}\right)$ causing a blue shift to the absorption spectrum with respect to the monomer spectrum, while the J-dimer has a head-to-tail arrangement $\left(\theta=180^{\circ}\right)$ causing a red shift to the absorption spectrum.$^{6-7}$

Fluorescence detection has an impressive high sensitivity. However, regardless the high sensitivity, fluorescence has a poor selectivity. To improve the selectivity of fluorescence an array of fluorescent sensing elements was used to target specific analytes and detect new molecules ${ }^{5,9-10}$. In previous work the fluorescence emission of Rhodamine $6 \mathrm{G}$ was amplified by 3 orders of magnitude using a simple layered structure. ${ }^{4-11}$ Also, it was reported that doping PVDF with a transition metal salt $\left(\mathrm{Zn}^{2+}\right)$ induced $\beta$-phase formation, increased the absorbance, enhanced the 
fluorescence emission and removed rhodamine $6 \mathrm{G}$ aggregations. This work will focus on studying the factors that led to the major absorbance and fluorescence enhancement in the presence of the metal ion and how the aggregation of Rh6G on the polymer surface was completely suppressed, even at a low doping level of $1 \mathrm{~mol} \% \mathrm{Zn}^{2+}$.

Dye molecules aggregation is a major source of decreasing the fluorescent emission. ${ }^{6-20}$ Therefore, in order to achieve high fluorescence intensity, a material of dye monomers should be prepared. To begin understanding the role of the transition metal salt on increase the absorbance, enhance the fluorescence emission and remove rhodamine 6G aggregation, the absorption and emission spectra of Rh6G on PMMA and PVDF cast from acetone were examined in more detail.

In chapter one, when PVDF polymer cast from (acetone/DMF) was doped with a few mole percent of zinc nitrate hexahydrate, the character of the absorbance spectra changed. As the films get thicker we do not see the shift in wavelength as in Rh6G on glass substrate and PVDF thin films. All the peaks have $\sim 520 \mathrm{~nm}$ maximum wavelengths with a linear relationship between the concentration of Rh6G and the zinc mol \%. At low Rh6G concentration there are two features, a peak at $\sim 520 \mathrm{~nm}$ and a shoulder at $\sim 480 \mathrm{~nm}$. At high $\mathrm{Rh} 6 \mathrm{G}$ concentrations the spectra have the same line shape with no peak growing at $550 \mathrm{~nm}$. The same effect is seen independent of the $\mathrm{Zn}^{2+}$ concentration, but the absorbance is significantly higher. This indicates that the presence of $\mathrm{Zn}^{2+}$ is stopping $\mathrm{Rh} 6 \mathrm{G}$ from aggregating to form the new absorbing species centered at $550 \mathrm{~nm}$. However, TGA and FTIR measurements also showed significant DMF retained in the films, coordinated to the $\mathrm{Zn}^{2+}$. 
The work reported here is to investigate the role of the polymer substrate, the solvent and potential in the actions between $\mathrm{Rh} 6 \mathrm{G}$ and $\mathrm{Zn}^{2+}$. Determination of a reaction between $\mathrm{Rh} 6 \mathrm{G}$ and $\mathrm{Zn}^{2+}$ is explored using absorption spectroscopy in the absence of a polymer. Next, films of PVDF doped with $\mathrm{Zn}^{2+}$ and cast from pure acetone to find the role of DMF. Finally, PMMA is used as the polymer substrate to evaluate the role of PVDF (Fig.1).

\section{EXPERIMENTAL}

\subsection{Film Materials}

Poly (methyl methacrylate) (PMMA) with a molecular weight $100.12 \mathrm{~g} / \mathrm{mol}$ was purchased from Sigma Aldrich. Polyvinylidene fluoride with a molecular weight $534,000 \mathrm{~g} / \mathrm{mol}$ was purchased from Sigma Aldrich. Zinc (II) nitrate hexahydrate salt with a molecular weight $297.47 \mathrm{~g} / \mathrm{mol}$ was purchased from Fisher Science Education. Spectral grade acetone and N, N-Dimethylformamide (DMF) solvents were purchased from Fisher Scientific. Glass slides were used as a substrate for film formation.

\subsection{Sample Preparation}

The glass slides were cut into dimensions of $3.75 \mathrm{~cm} \times 1.75 \mathrm{~cm}$. The slides were placed in a container of $95 \%$ ethanol $(\mathrm{EtOH})$ and sonicated for 15 minutes. Then they were rinsed three times and placed in distilled water and sonicated for an additional 15 minutes, then dried with $\mathrm{N}_{2}$ gas. ${ }^{12}$ A solvent system of 90/10 volume /volume of acetone to N, N-Dimethylformamide (DMF) was used for dissolution of $3 \%$ weight /volume ratio of PVDF polymer to overall volume of solvent. ${ }^{6}$

A solvent system of $90 / 10$ volume /volume of acetone to $\mathrm{N}, \mathrm{N}$ Dimethylformamide (DMF) was used for dissolution of 3\% weight /volume ratio of 
PVDF polymer to overall volume of solvent. ${ }^{13}$ The polymer solution was placed in a Branson 3510 ultrasonic cleaning device for 3 hours in a $40^{\circ} \mathrm{C}$ bath to ensure polymer was dissolved. Specified mole percentages of zinc (II) determined the mass of zinc (II) nitrate hexahydrate salt that was added to the polymer solution after the sonication process, using only a mechanical swirling technique to dissolve the salt and an additional 2 to 3 minutes sonication time was allowed, to ensure that the salt was fully dissolved in solution, and the calculated mass percentage was accurate. Once the polymer is dissolved in solution, films were made. Using a Laurell Technologies WS400B-6NPP/LITE spin coating device and a glass slide as substrate, the solution is deposited on the glass slide with a $300 \mu \mathrm{L}$ volume at $1200 \mathrm{RPM}$ for 45 seconds with an acceleration of $1080 / s^{2}$. After spin casting of polymer solutions was complete, the samples were then placed in an oven set to $60^{\circ} \mathrm{C}$ for 2 minutes to dry. A series of Rhodamine $6 \mathrm{G}$ concentrations were made in EtOH ranging from $1 \times 10^{-4} \mathrm{M}-$ $9 \times 10^{-4} \mathrm{M}$. The glass vials containing the solution was wrapped in aluminum foil to prevent absorbing light. The Rhodamine 6G solution was also spun-cast in a similar manner. A volume of $50 \mu \mathrm{L}$ at $1200 \mathrm{RPM}$ for 45 second with an acceleration of $1080 / s^{2}$ of Rh6G solution was deposited on the sample and left to dry.

\section{MEASUREMENTS}

Perkin Elmer Lambda 1050 UV-Visible spectrometer was employed for obtaining UV-Vis spectra. Before the absorbance spectrums were collected, a blank of clean glass slide was taken. A Horiba (JobinYvon) Fluorolog spectrometer was used for steady-state fluorescence measurements and data were collected at a 60-degree angle. The emission wavelength range was from 520 to $800 \mathrm{~nm}$ with a slit width of 3.0 
$\mathrm{nm}$. The excitation wavelength range was from $300 \mathrm{~nm}$ to $800 \mathrm{~nm}$ with a slit width of $2.0 \mathrm{~nm}$ to correspond with the absorbance spectrum.

\section{RESULT AND DISCUSSION}

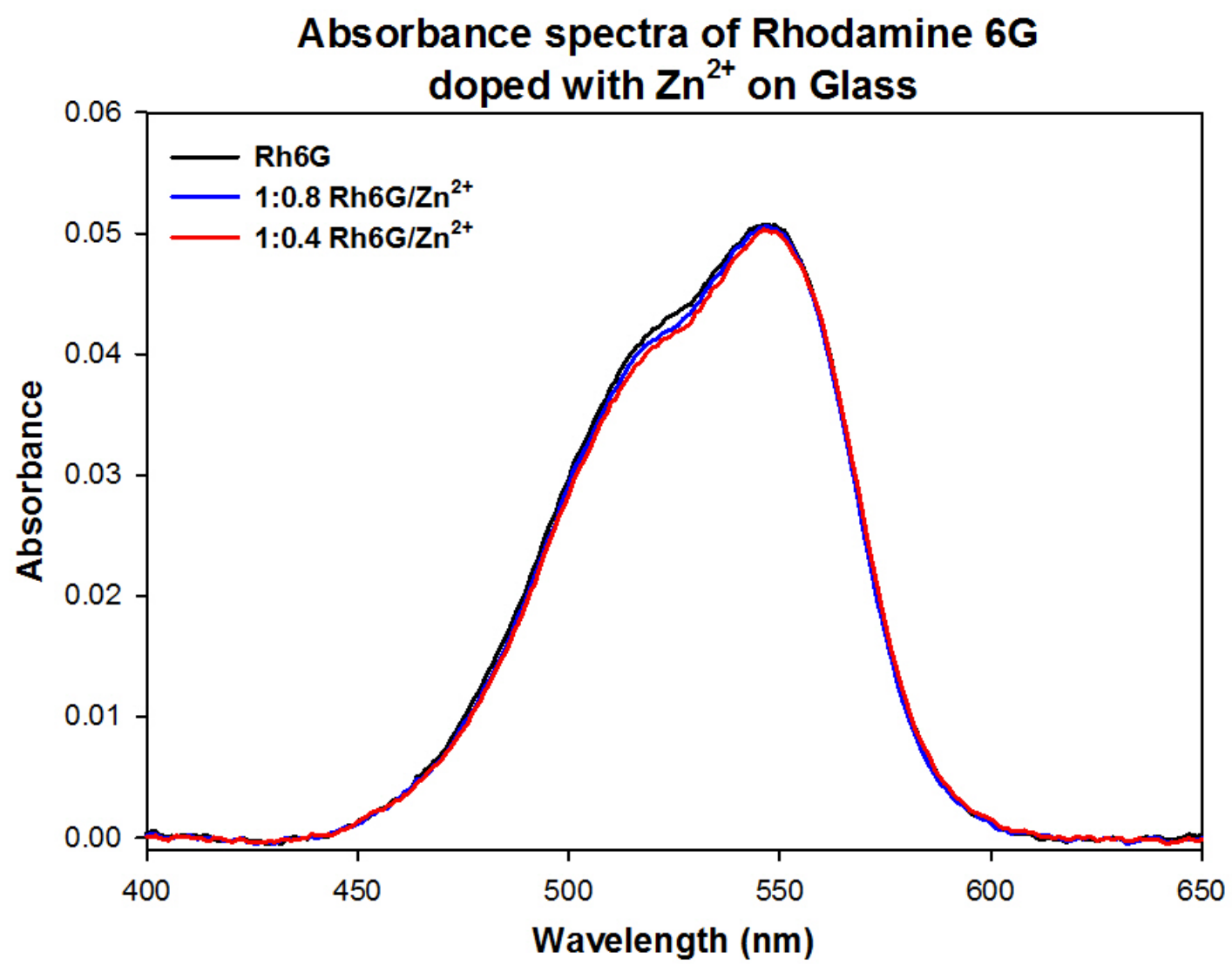

Figure 1. Absorbance Spectra of $9 \times 10^{-4} \mathrm{M}$ Rh6G Doped with $\mathrm{Zn}^{2+}$ on Glass Slide.

The second hypothesis was the DMF solvation influence, in order to examine if that behavior was due to the DMF solvation, an experiment was conducted where a pure acetone was used as a solvent. It has been found from the optical absorbance spectra that using acetone solvation in $\mathrm{PVDF} / \mathrm{Zn}^{2+}$ thin films doped with $\mathrm{Rh} 6 \mathrm{G}$ has an absorbance maximum at $\sim 520 \mathrm{~nm}$ and the shoulder on the red side at $\sim 550$ was removed (Fig.2 and Fig.3). 


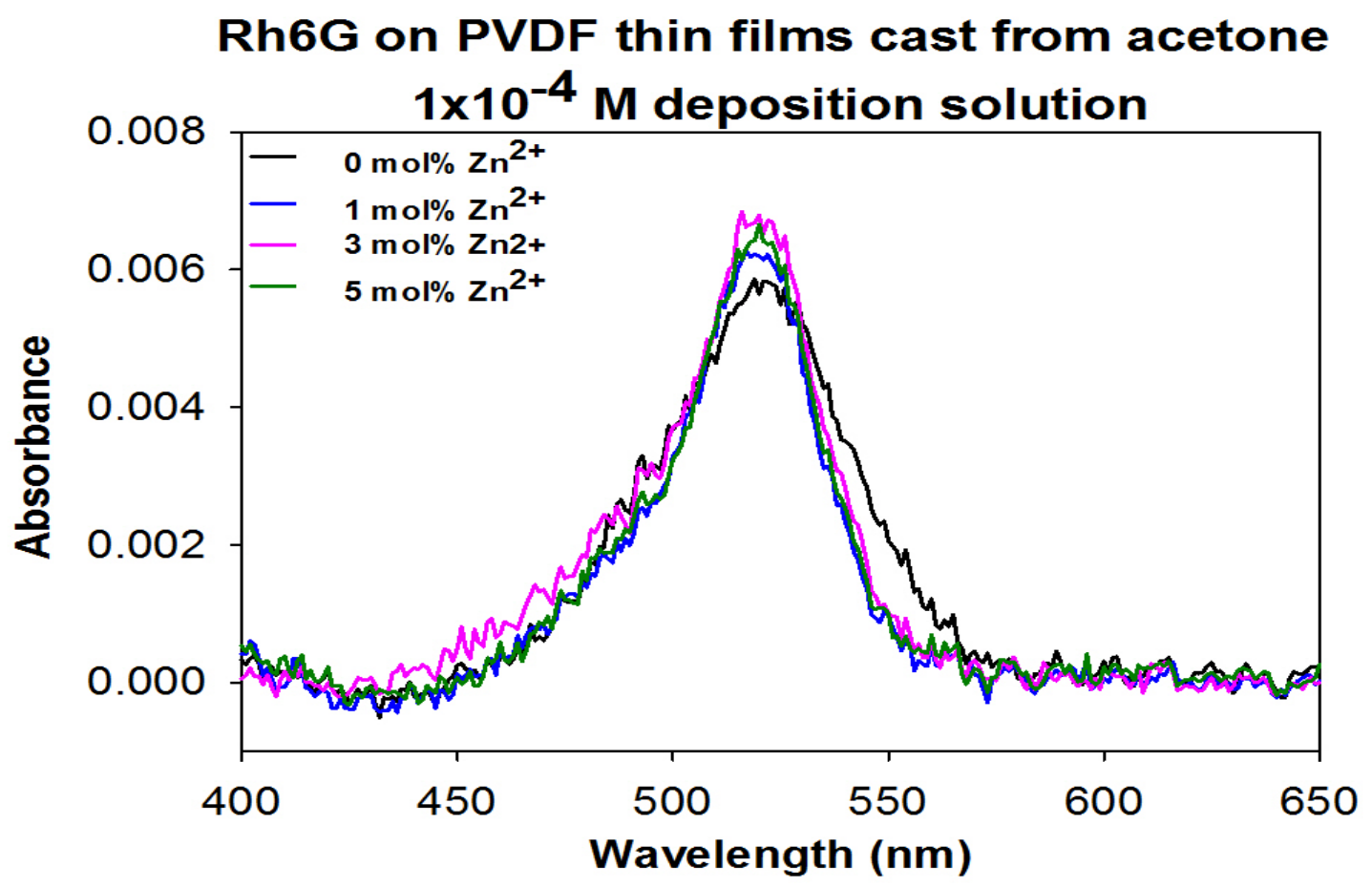

Figure 2. Absorbance spectra of $1 \times 10^{-4} M$ Rh6G on PVDF thin films cast from acetone as a function of $\mathrm{Zn}^{2+} \mathrm{mol} \%$.

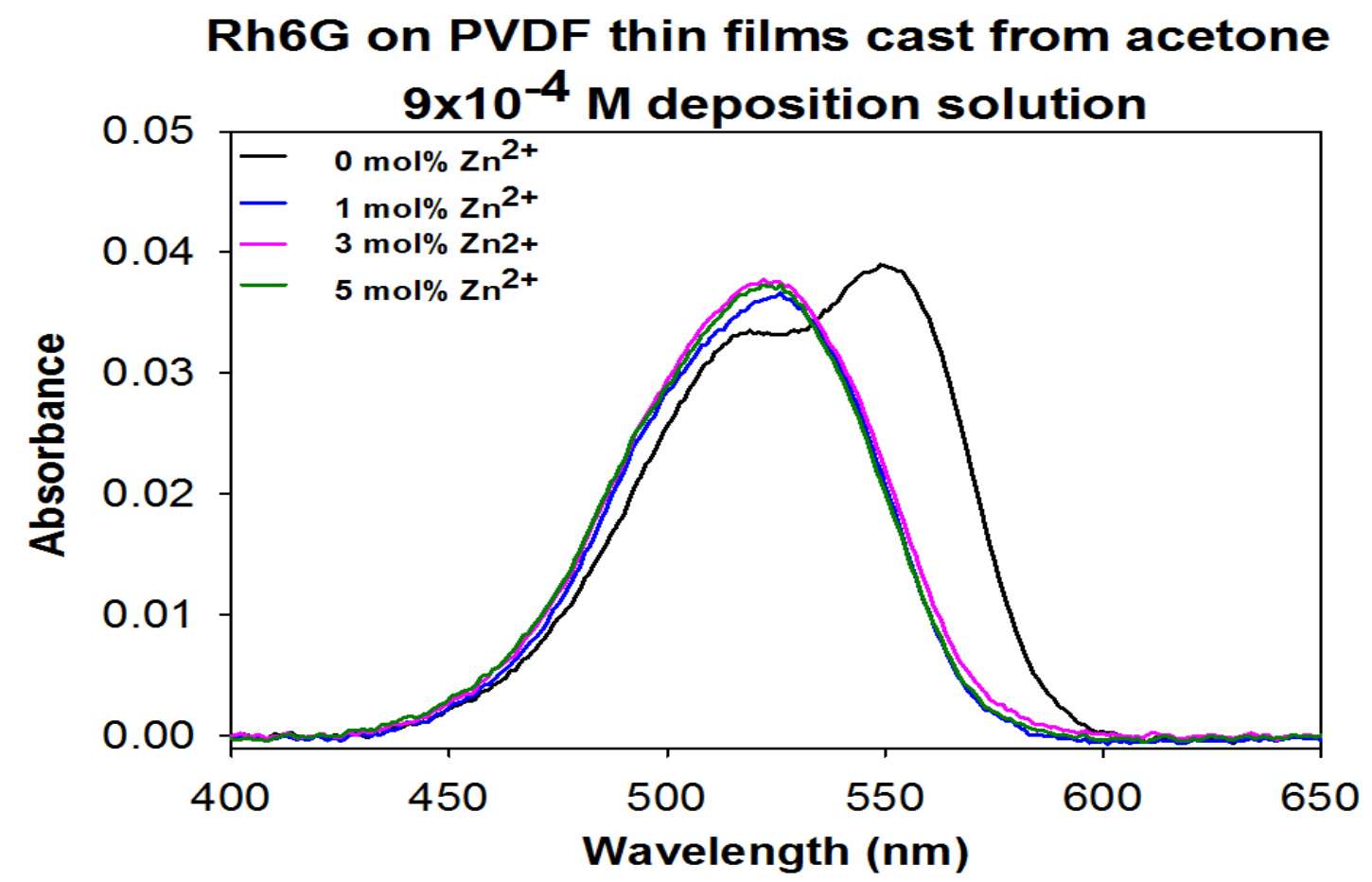

Figure 3. Absorbance spectra of $9 x 10^{-4} M$ Rh6G on PVDF thin films cast from acetone as a function of $\mathrm{Zn}^{2+} \mathrm{mol} \%$. 
However, the absorbance maxima has a very small different as a function of the zinc mol\% unlike the DMF solvation. This experiment indicates the zinc metal ion is the causative factor of removing the dye molecular aggregation.

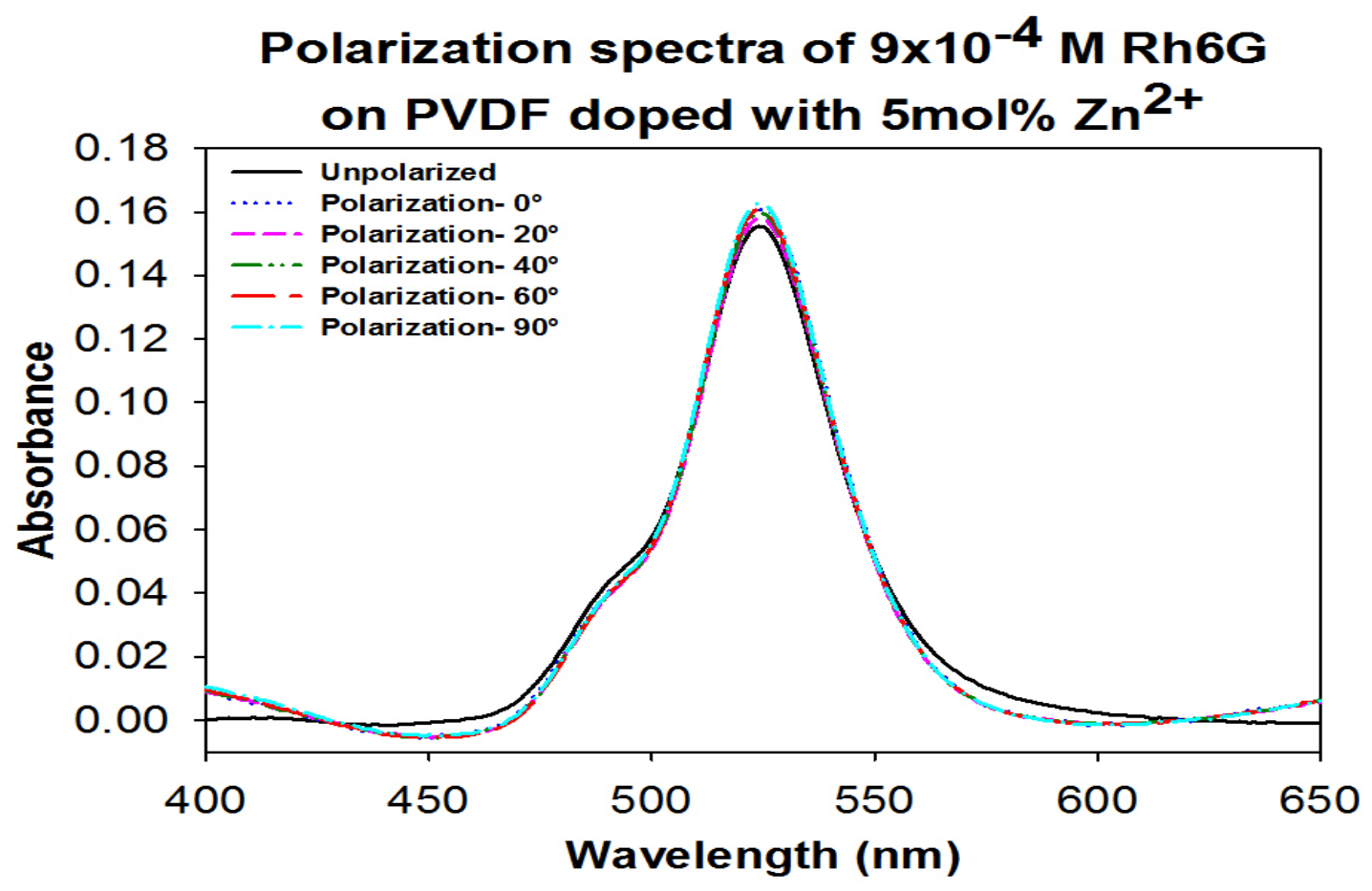

Figure 4. Polarization spectra of $9 \times 10^{-4} \mathrm{M}$ Rh6G on PVDF doped with $5 \mathrm{~mol} \%$ $\mathrm{Zn}^{2+}$.

Another fascinating result is that as the zinc ion increases in the film, the absorbance is increased as well at the same [Rh6G]. In the first place, this was attributed to the continuous enhancement in the maximum absorbance intensity to Rh6G fluorophore orientation on the surface. It was though that in the presence of zinc metal ion the dye orientation would change facing the light, which would cause increasing in the absorbance intensity as the percentage of the metal ion was increased at the same concentration of Rh6G. However, the polarization experiment showed a random structure of the dye, since all of the peaks are overlapped no matter what value of polarization angle was used (Fig 4). 
The third hypothesis was the presence of $\beta$-phase PVDF; to test this the PVDF polymer was replaced with PMMA.

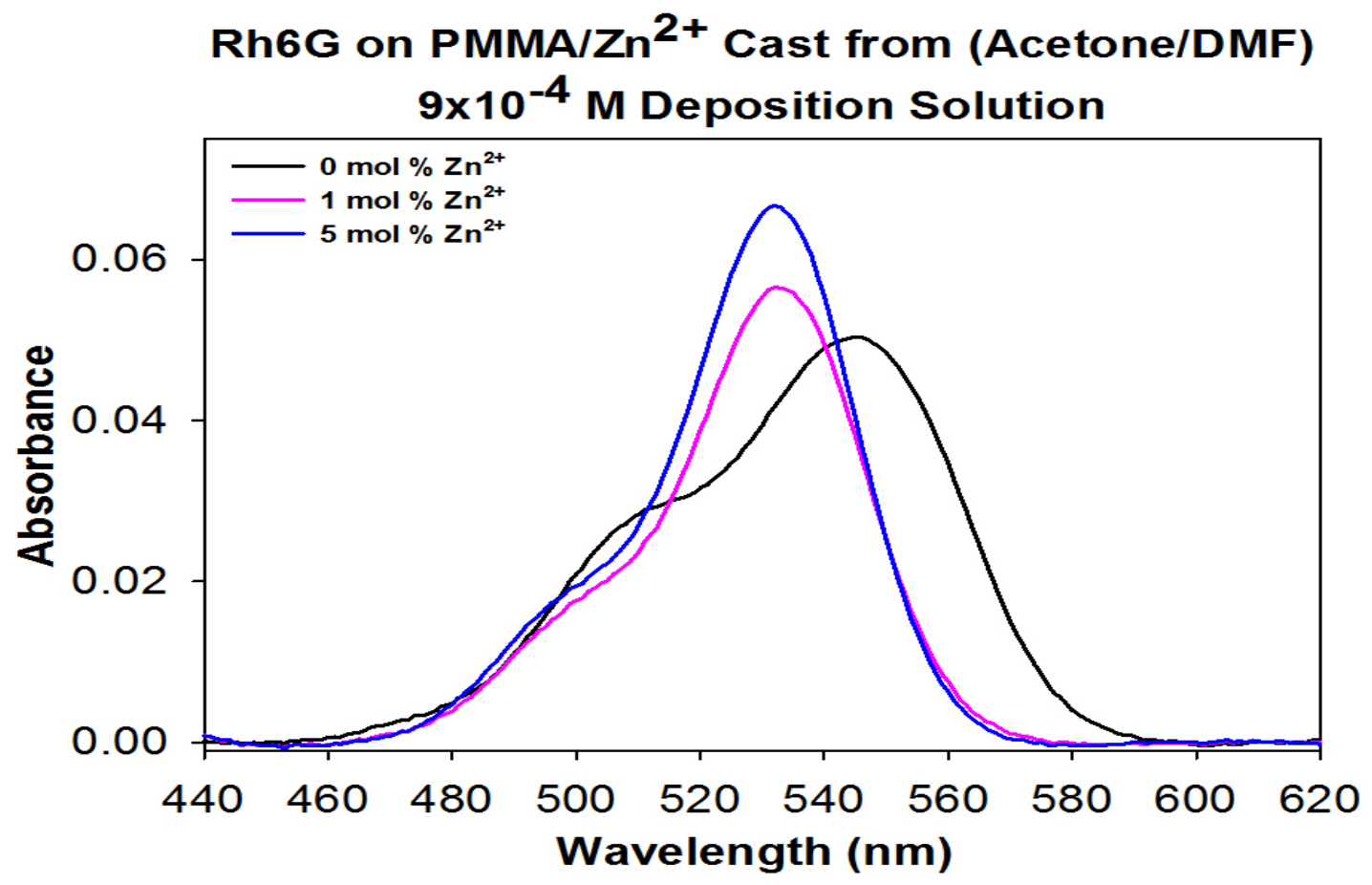

Figure 5. Absorbance spectra of $9 \times 10^{-4} \mathrm{M}$ Rh6G on PMMA/ $\mathrm{Zn}^{2+}$ thin films as a function of $\mathrm{Zn}^{2+} \mathrm{mol} \%$.

Fig.5 shows an additional support to the major influence of zinc metal ions in removing the dye molecular aggregation. A single peak appears at $\sim 550 \mathrm{~nm}$ with a shoulder at $\sim 510 \mathrm{~nm}$ when PMMA thin film was doped with a high Rh6G concentration $\left(9 \times 10^{-4} \mathrm{M}\right)$. However, doping $\mathrm{PMMA} / \mathrm{Zn}^{2+}$ with the same concentration of $\mathrm{Rh} 6 \mathrm{G}$ has a single peak at $\sim 530 \mathrm{~nm}$.

To demonstrate that the emission enhancement was due to the presence of $\mathrm{Zn}^{2+}$ and not the DMF solvation, an experiment was conducted using acetone solvation. 


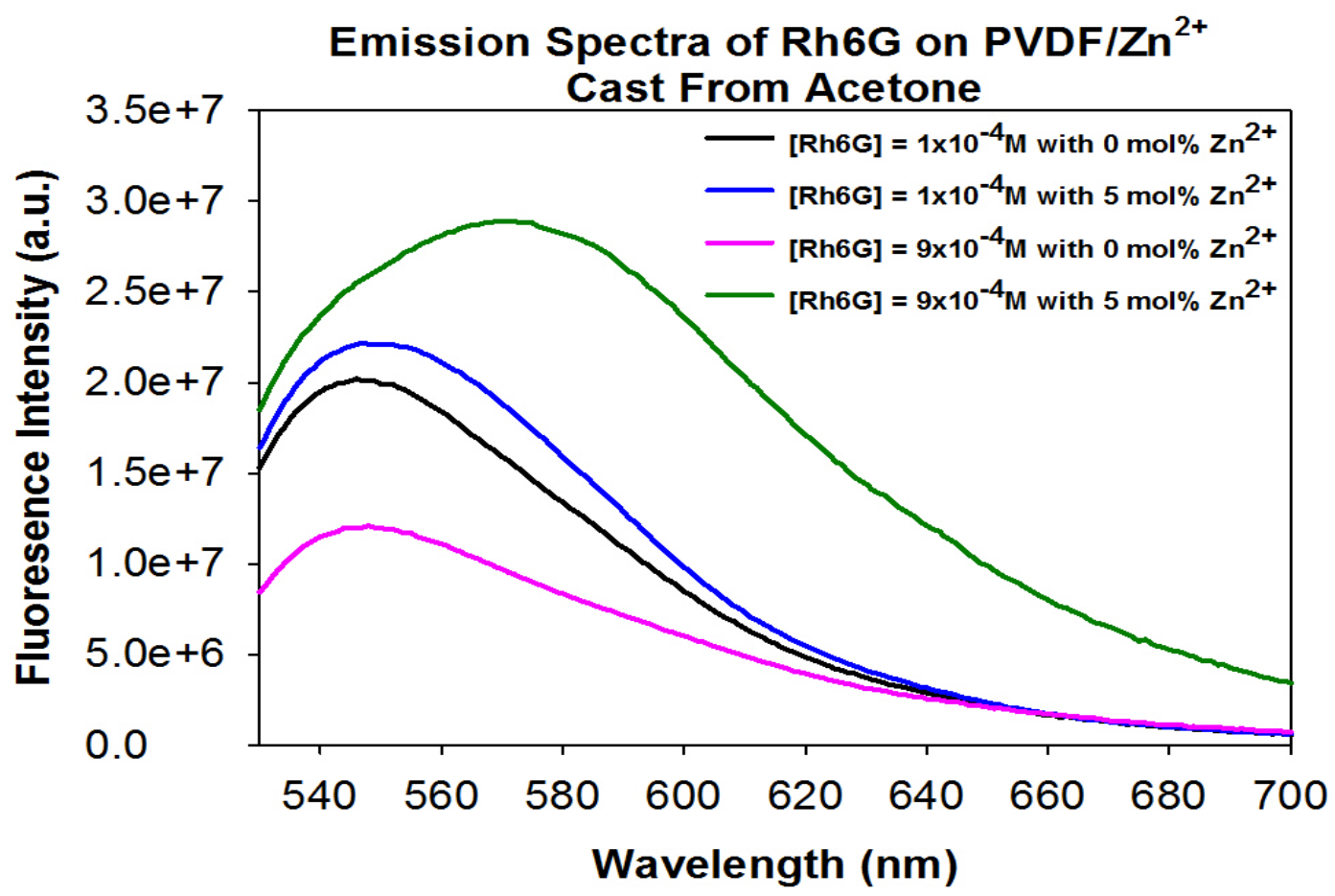

Figure 6. Emission spectra of [Rh6G] on PVDF in acetone thin films as a function of $\mathrm{Zn}^{2+} \mathrm{mol} \%$.

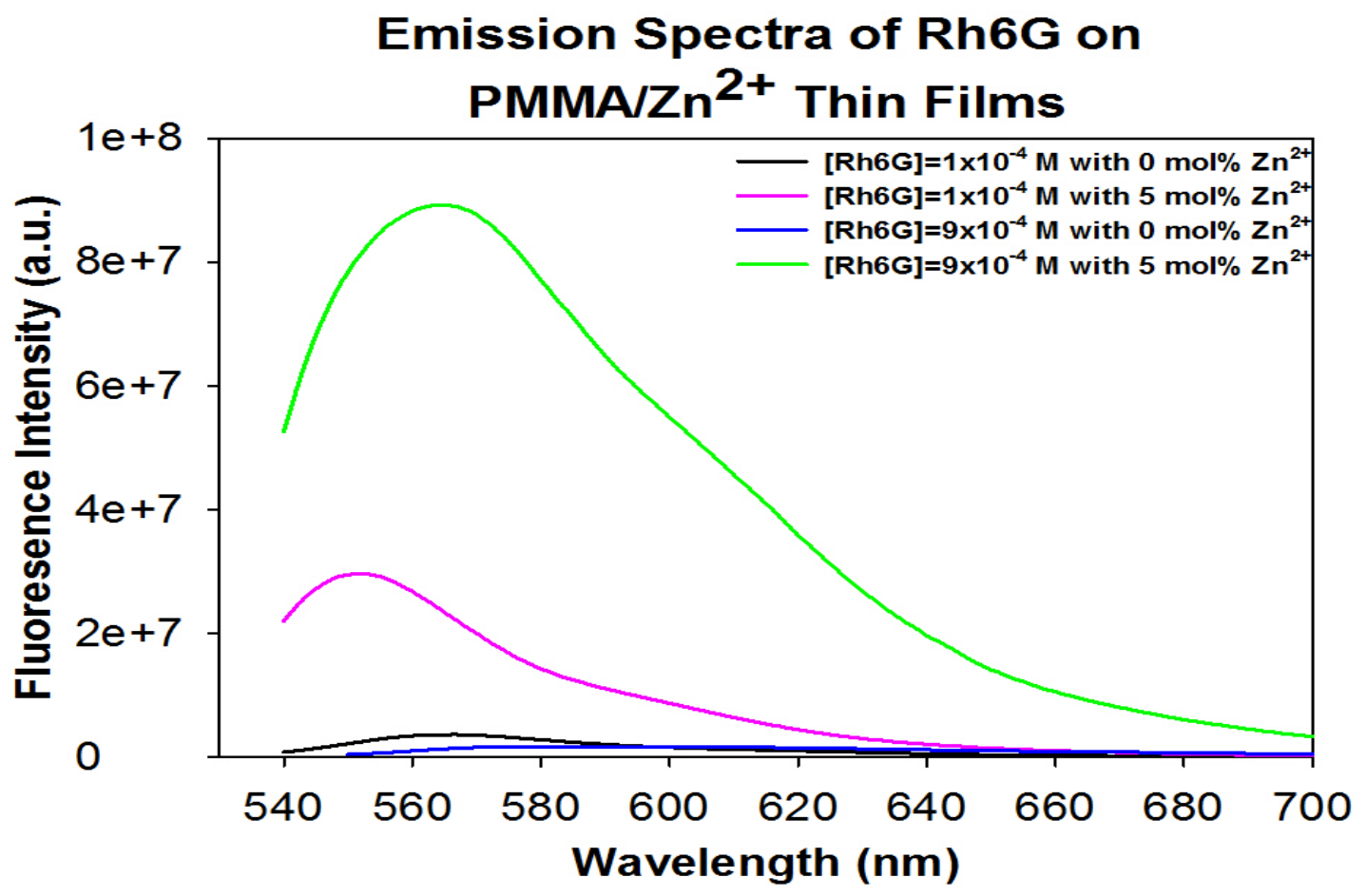

Figure 7. Emission spectra of $[\mathrm{Rh} 6 \mathrm{G}]$ on PMMA thin films as a function of $\mathrm{Zn}^{2+} \mathrm{mol} \%$. 
In Fig. 6 an emission spectra of $\mathrm{PVDF} / \mathrm{Zn}^{2+}$ thin films was compared with a native PVDF films cast from acetone solvation. It shows that the presence of $\mathrm{Zn}^{2+}$ has an increase in the emission intensity by $1.7 \times 10^{7} \mathrm{a}$. u. at high concentration of Rh6G. This is another support to the hypothesis for the strong influence of zinc metal ions in removing the dye molecules aggregation, and enhances the emission intensity. Fig.7 shows an emission enhancement in the PMMA/ $\mathrm{Zn}^{2+}$ thin films compared with the native PMMA films cast from (DMF/Acetone) by $8.84 \times 10^{7}$ a. u. at high concentration of $\mathrm{Rh} 6 \mathrm{G}$, and this is in agreement with the previous hypothesis.

\section{CONCLUSION}

PVDF polymer cast from DMF/acetone mixture doped with a few mole percent of zinc nitrate hexahydrate increases the production of the ferroelectric $\beta$ phase, emission intensity and the absorbance maxima. PVDF cast from acetone doped with $\mathrm{Zn}^{2+}$ removed the aggregation. However, the absorbance maximum did not change.

PMMA polymer cast from DMF/acetone mixture doped with $\mathrm{Zn}^{2+}$ increases the absorbance maximum with no aggregation. The previous result supports that the presence of $\mathrm{Zn}^{2+}$ metal ion removes the aggregation. The $\mathrm{DMF} / \mathrm{Zn}^{2+}$ mixture increases the absorbance maximum as a function of $\mathrm{Zn}^{2+}$ mole percent.

In a conclusion, $\mathrm{Zn}^{2+}$ suppresses aggregation even at high [Rh6G] and emission enhancement still occurs. DMF causes absorption changes.

A number of experiments were conducted in order to understand the role of the interface on the fluorophore/polymer properties. UV-Visible spectroscopy was used to 
determine if the fluorophore is in a monomer or a dimers structure. This is especially important to determine the role of the fluorescence enhancement (see Table 1).

Table 1. The Response of Rh6G on different substrates.

\begin{tabular}{|c|c|c|c|c|c|}
\hline $\begin{array}{c}\text { Response of } \\
\text { Rh6G } \\
\text { thickness }\end{array}$ & Rh6G/Glass & Rh6G/PVDF & $\begin{array}{c}\text { Rh6G/ PVDF/Zn' } \\
\text { (Acetone/DMF) }\end{array}$ & $\begin{array}{c}\text { Rh6G/ } \\
\mathbf{P V D F} / \mathbf{Z n}^{2+} \\
\text { (Acetone) }\end{array}$ & $\begin{array}{c}\text { Rh6G/ } \\
\text { PMMA/Zn }\end{array}$ \\
\hline$A_{\max }$ & $\begin{array}{c}\text { Non linear } \\
\text { increase }\end{array}$ & $\begin{array}{c}\text { Non linear } \\
\text { increase }\end{array}$ & Linear increase & $\begin{array}{c}\text { Linear } \\
\text { increase }\end{array}$ & $\begin{array}{c}\text { Linear } \\
\text { increase }\end{array}$ \\
\hline $\begin{array}{c}\lambda_{\max } \\
\left(\begin{array}{c}\text { Absorptio } \\
\text { n) }\end{array}\right.\end{array}$ & Red shift & Red shift & No shift & No shift & No shift \\
\hline$I_{\max }$ & Decrease & Decrease & Increase & Increase & Increase \\
\hline $\begin{array}{c}\lambda_{\max } \\
(\text { Emission })\end{array}$ & Red shift & Red shift & $\begin{array}{c}\text { Recon shift } \\
\text { reduce }\end{array}$ & Red shift & Red shift \\
\hline $\begin{array}{c}\text { Aggregati } \\
\text { on }\end{array}$ & Yes & Yes & No & No & No \\
\hline
\end{tabular}




\section{REFERENCES}

1. J-S. Yang and T.M. Swager, "Fluorescent Porous Polymer Films as TNT Chemosensors: Electronic and Structural Effects," J. Am. Chem. Soc., 1998, $120,11864-11873$.

2. B. D. de Greñu, D. Moreno, T. Torroba, A. Berg, J. Gunnars, T. Nilsson, R. Nyman, M. Persson, J. Pettersson, I. Eklind, and P. Wästerby, "Fluorescent Discrimination between Traces of Chemical Warfare Agents and Their Mimics,” J. Am. Chem. Soc., 2014, 136, 4125-4128.

3. I. A. Levitsky, W. B. Euler, N. Tokranova, A. Rose, "Fluorescent Polymer Porous Silicon Microcavity Devices for Explosives Detection," Appl. Phys. Lett., 2007, 90, 041904-1 - 041904-3.

4. M. A. Matoian, R. Sweetman, E. C. Hall, S. Albanese, W. B. Euler, "Light Trapping to Amplify Metal Enhanced Fluorescence with Application for TNT Sensing," J. Fluoresc., 2013, 23, $877-880$.

5. C. A. Latendresse, S.C. Fernandes, S. You, H. Q. Zhang, W. B. Euler, "A Fluorometric Array for the Detection of Military Explosives and IED Materials," Anal. Methods, 2013, 5, $5457-5463$.

6. F.P. Schafer, “ Topics in Applied Physics,” Vol. 1 (Springer, Berlin, 1973).

7. E. G. McRae and M. Kasha, "The enhancement of phosphorescence ability upon aggregation of dye molecules, “ J. Chem. Phys. 28, 721 (1958).

8. K. Kemnitz and K. Yoshiara," Entropy-driven dimerization of xanthene dyes in nonpolar solution and temperature-dependent fluorescence decay of dimers," J. Phys. Chem. 95, 6095 (1991).

9. W. Xu, C. Ren, C. L. Teoh, J. Peng, S. H. Gadre, H.-W. Rhee, C.-L. K. Lee, and Y.-T. Chang, "An Artificial Tongue Fluorescent Sensor Array for Identification and Quantitation of Various Heavy Metal Ions," Anal. Chem., 2014, 86, 8763 - 8769 .

10. S. Wang, L. Ding, J. Fan, Z. Wang, and Y. Fang, "Bispyrene/SurfactantAssembly-Based Fluorescent Sensor Array for Discriminating Lanthanide Ions in Aqueous Solution," ACS Appl. Mater. Interfaces, 2014, 6, 16156- 16165.

11. Benz, M., Euler, W.B. Langmurir, 2001.17, 239-243.

12. The Role of Solution Phase Water on the Deposition of Thin Films of Poly(vinylidene fluoride)," M. Benz, W. B. Euler, O. J. Gregory, Macromolecules, 2002, 35, 2682-2688. 
13. M. Benz and W. B. Euler, "Determination of the Crystalline Phases of Poly(vinylidene fluoride) Under Different Preparation Conditions Using Differential Scanning Calorimetry and Infrared Spectroscopy,” J. Appl. Polym. Sci., 2003, 89, 1093-1100.

14. K. Fontaine, W. Lopez, E. Crisman, J. Derov, and W. B. Euler, "Doping of Polyvinylidene Difluoride with Cobalt Nitrate: Structural, Electrical, and Magnetic Properties," J. Polym. Sci. A: Polym. Chem., 2012, 50, 3970 - 3875.

15. J. R. Lakowicz, Principles of Fluorescence Spectroscopy, 3rd edition, Springer Science, 2006.

16. A. Satrijo, S. E. Kool, and T. M. Swager, "Enhanced Luminescence from Emissive Defects in Aggregated Conjugated Polymers," Macromolecules, 2007, 40, $8833-8841$.

17. A. Qin, J. W. Y. Lam, L. Tang, C. K. W. Jim, H. Zhao, J. Sun, and B. Z. Tang, "Polytriazoles with Aggregation-Induced Emission Characteristics: Synthesis by Click Polymerization and Application as Explosive Chemosensors," Macromolecules, 2009, 42, 1421 - 1424.

18. W. Z. Yuan, H. Zhao, X. Y. Shen, F. Mahtab, J. W. Y. Lam, J. Z. Sun, and B. Z. Tang, "Luminogenic Polyacetylenes and Conjugated Polyelectrolytes: Synthesis, Hybridization with Carbon Nanotubes, Aggregation-Induced Emission, Superamplification in Emission Quenching by Explosives, and Fluorescent Assay for Protein Quantitation," Macromolecules, 2009, 42, 9400 -9411 .

19. J. Liu, Y. Zhong, J. W. Y. Lam, P. Lu, Y. Hong, Y. Yu, Y. Yue, M. Faisal, H. H. Y. Sung, I. D. Williams, K. S. Wong, and B. Z. Tang, "Hyperbranched Conjugated Polysiloles: Synthesis, Structure, Aggregation-Enhanced Emission, Multicolor Fluorescent Photopatterning, and Superamplified Detection of Explosives," Macromolecules, 2010, 43, 4921 - 4936.

20. P. Innocenzi, H. Kozuka, T. Yoko, Dimer-to-monomer transformation of rhodamine $6 \mathrm{G}$ in sol-gel silica films, Journal of Non-Crystalline Solids, 1996, 201, 1-2. 


\title{
CHAPTER 3
}

\section{Detection of Gas-Phase Explosive Analytes Using Fluorescent Spectroscopy of PVDF Doped with Rhodamine 6G}

\author{
Mona Alhasani, ${ }^{1}$ William B. Euler ${ }^{1 *}$ \\ 1. Department of Chemistry, University of Rhode Island, 140 Flagg Road, Kingston, \\ RI 02881 \\ * Corresponding Authors weuler@chm.uri.edu, phone 401-874-5090, fax 401-874- \\ 5072 .
}

The following is in preparation for submission to the Journal of Langmuir (ACS), and is presented here in manuscript format. 
ABSTRACT

The response of emission signal of TNT, as a function of Rhodamine 6G concentration and mol $\%$ of $\mathrm{Zn}^{2+}$ has been studied. Using a different concentration of Rhodamine $6 \mathrm{G}$ and mol \% of metal ion, the signal is quenched by a notably large amount. Different analytes can be applied and emission signals can be collected to find a pattern that could be used to identify the explosives. 


\section{INTRODUCTION}

Developing a sensor device to detect explosives is an active area of research for the past decades. ${ }^{1-56}$ The reliable detection of explosives is an international concern and it is important for homeland security, environmental safety and the military. While there are many suitable methods for the detection of explosives, we have been using fluorescence as our technique because it has the benefit of sensitivity, low cost and frequently used for explosive sensors. An example of an effective explosive sensor was developed by Dr. Swager research group using a substrate with a conjugated fluorescent polymer. ${ }^{57}$ Since then an increasing interest has been paid to the detection of explosives using a fluorescent amplifying polymer technique. ${ }^{58,59}$

In chapter one, Fluorescent enhancements have been achieved using a simple layered structure: fluorophore, Polymer/metal ion, glass substrate. The polymer/metal ion layer had a strong influence on the emission response of the fluorophore by removing the dye aggregation. The achieved fluorescent enhancements by the layered structure can be used for the detection of explosives. ${ }^{52-54}$ The idea behind fluorescent detection of explosives is measuring the emission signal intensity before and after applying the explosive to the sample: fluorophore (Rh6G), $\mathrm{PVDF} / \mathrm{Zn}^{2+}$, glass substrate. The change in the signal intensity is either quenching or increasing. In our case the signal is quenching by a notably large amount. When the explosive molecule collides with Rhodamine $6 \mathrm{G}$ in the excited state, more nonradiative pathways become available. Rhodamine 6G loses its energy and the amount of emitted light decreases and that leads to a decrease in the fluorescent signal. ${ }^{1,4,7,17,49,56}$ Then the amount of signal loss or gain can be used to identify the analyte. 
In order to find a pattern that can be used to distinguish the different explosives, a table can be made with difference emission spectra after exposure to various analytes: TNT, trinitrotoluene; TNB, trinitrobenzene; PETN, pentaerythritol tetranitrate; RDX, TATP, triacetone triperoxide; DNT, dinitrotoluene; NT, nitrotoluene. In this work, TNT, trinitrotoluene has been used as the analyte and a large quenching in the emission signal has been observed.

\section{EXPERIMENTAL}

\subsection{Film Materials}

Polyvinylidene fluoride with a molecular weight 534,000 g/mol was purchased from Sigma Aldrich. Zinc (II) nitrate hexahydrate salt with a molecular weight 297.47 g/mol was purchased from Fisher Science Education. Spectral grade acetone and N, N-Dimethylformamide (DMF) solvents were purchased from Fisher Scientific. Glass slides were used as a substrate for film formation. Trinitrotoluene (TNT) was purchased from Sigma Aldrich.

\subsection{Sample Preparation}

The glass slides were cut into dimensions of $3.75 \mathrm{~cm} \times 1.75 \mathrm{~cm}$. The slides were placed in a container of $95 \%$ ethanol $(\mathrm{EtOH})$ and sonicated for 15 minutes. Then they were rinsed three times and placed in distilled water and sonicated for an additional 15 minutes, then dried with $\mathrm{N}_{2}$ gas. ${ }^{60}$ A solvent system of 90/10 volume /volume of acetone to N, N-Dimethylformamide (DMF) was used for dissolution of $3 \%$ weight /volume ratio of PVDF polymer to overall volume of solvent. ${ }^{61} \mathrm{~A}$ solvent system of 90/10 volume /volume of acetone to N, N-Dimethylformamide (DMF) was used for dissolution of 3\% weight /volume ratio of PVDF polymer to overall volume 
of solvent. ${ }^{62}$ The polymer solution was placed in a Branson 3510 ultrasonic cleaning device for 3 hours in a $40^{\circ} \mathrm{C}$ bath to ensure all polymer dissolved. Specified mole percentages of Zinc (II) determined the mass of Zinc (II) nitrate hexahydrate salt that was added to the polymer solution after the sonication process, using only a mechanical swirling technique to dissolve the salt and an additional 2 to 3 minutes sonication time was allowed, to ensure that the salt was fully dissolved in solution, and the calculated mass percentage was accurate. Once the polymer is dissolved in solution, films can be made. Using a Laurell Technologies WS-400B-6NPP/LITE spin coating device and a glass slide as substrate, the solution is deposited on the glass slide with a $300 \mu \mathrm{L}$ volume at 1200 RPM for 45 seconds with an acceleration of $1080 / s^{2}$. After spin casting of polymer solutions was complete, the samples were then placed in an oven set to $60^{\circ} \mathrm{C}$ for 2 minutes to dry. A series of Rhodamine $6 \mathrm{G}$ concentrations were made in EtOH ranging from $1 \times 10^{-4} \mathrm{M}-9 \times 10^{-4} \mathrm{M}$. The glass vials containing the solution was wrapped in aluminum foil to prevent absorbing light. The Rhodamine $6 \mathrm{G}$ solution was also spun-cast in a similar manner. A volume of $50 \mu \mathrm{L}$ at $1200 \mathrm{RPM}$ for 45 seconds with an acceleration of $1080 / s^{2}$ of Rh6G solution was deposited on the sample and left to dry.

The prepared sample was then placed in the Fluorometer and flushed with vapor TNT explosive for one minute and the flouresnce emission measurements were taken. The same process was repeated for different amounts of time. All of these measurements were done without moving the samples in order to guarantee that the same spot in the samples where measured. 


\section{MEASUREMENTS}

A Horiba (JobinYvon) Fluorolog spectrometer was used for steady-state fluorescence measurements and data were collected at a 60-degree angle. The emission wavelength range was from 520 to $800 \mathrm{~nm}$ with a slit width of $3.0 \mathrm{~nm}$. The excitation wavelength range was from $300 \mathrm{~nm}$ to $800 \mathrm{~nm}$ with a slit width of $2.0 \mathrm{~nm}$ to correspond to the absorbance spectrum.

\section{RESULT AND DISCUSSION}

In this section, the response of the emission signal of TNT, trinitrotoluene as a function of Rhodamine $6 \mathrm{G}$ concentration and mol \% of Zinc (II) has been studied. At different concentrations of the fluorophore and mol \% of metal ion, the signal is quenched by a notably large amount. This might be caused by the analyte interacting with the fluorophore to cause collisional quenching. 

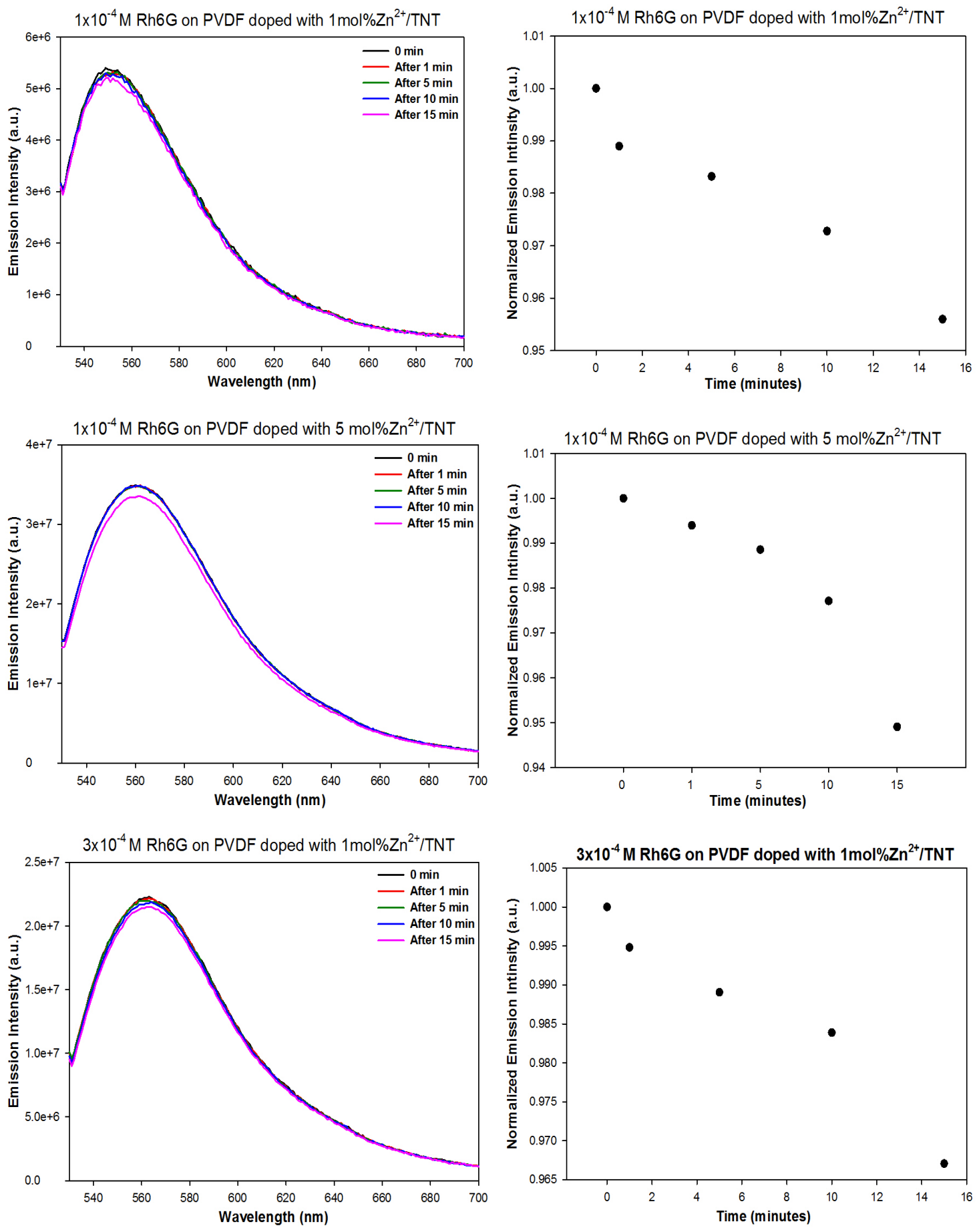

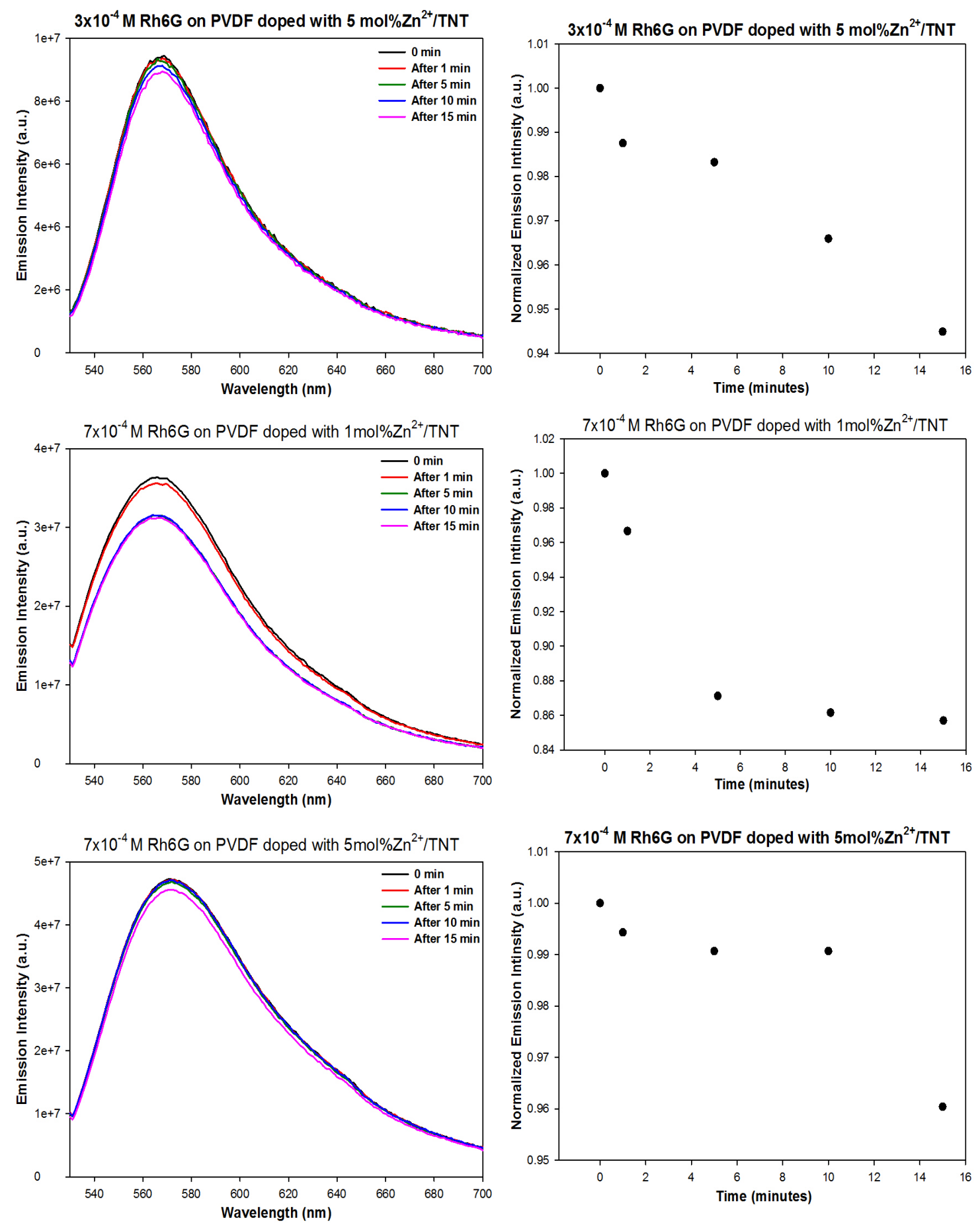

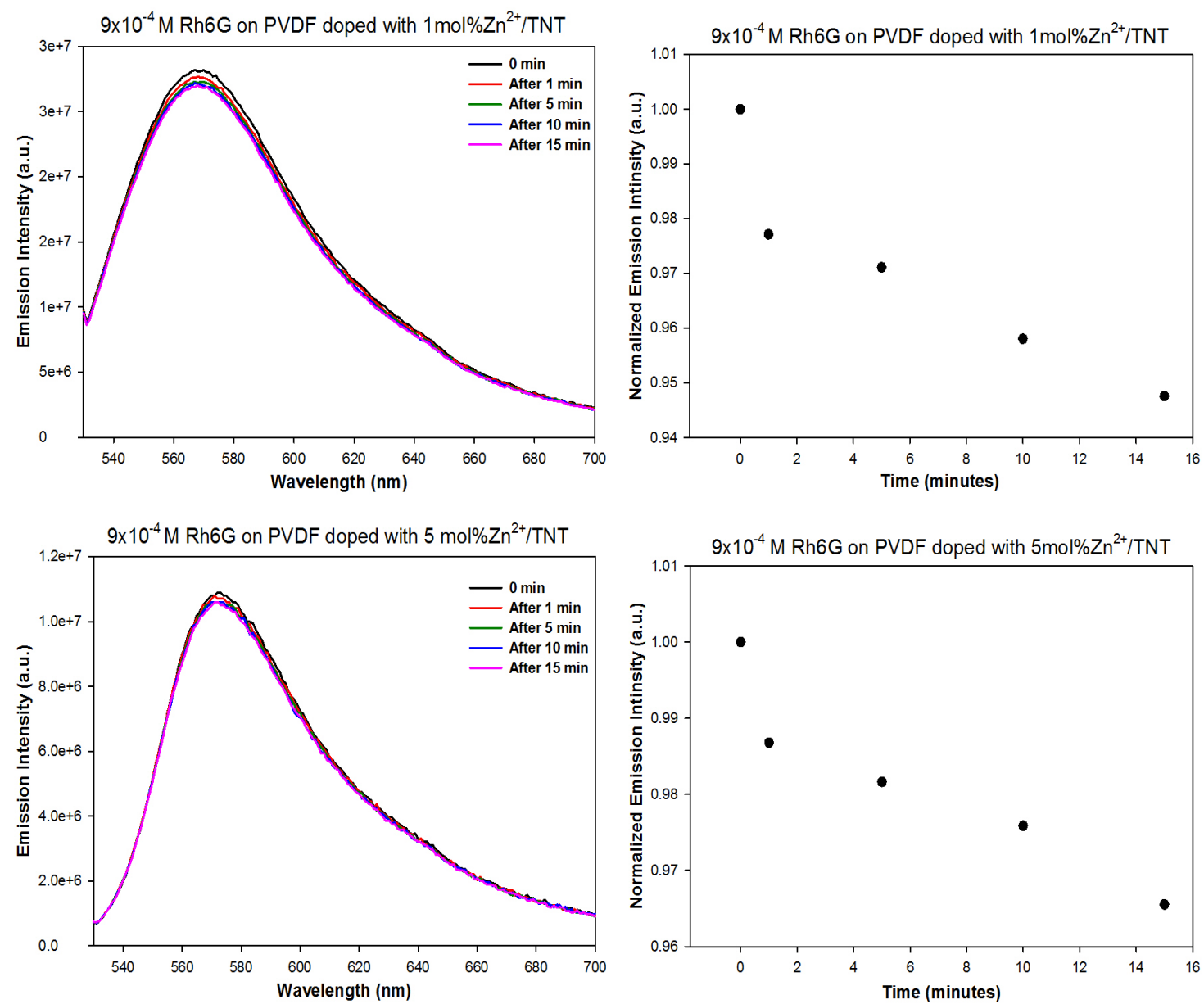

Figure 1. Emission spectra of different concentrations of Rh6G on PVDF surfaces with different $Z n^{2+}$ doping levels after exposure to TNT.

Figure 1 shows the emission spectra of different concentrations of Rh6G $\left(1 \times 10^{-4} \mathrm{M}, 3 \times 10^{-4} \mathrm{M}, 7 \times 10^{-4} \mathrm{M}\right.$ and $\left.9 \times 10^{-4} \mathrm{M}\right)$ on PVDF surfaces with different $\mathrm{Zn}^{2+}$ doping levels ranging from 1 to $5 \mathrm{~mol} \%$ after exposure to TNT. The scatter graphs show a large decrease in the emission signal after exposure to the analyte over time. Increasing the emission signal after exposure to the analyte could be caused by the breaking of aggregates by the analyte. 


\section{CONCLUSION}

In conclusion, the emission signal quenched by a notably large amount after adding TNT, trinitrotoluene to the layered structure: fluorophore, Polymer/metal ion, glass substrate. A table with different concentrations of Rhodamine $6 \mathrm{G}$ and $\mathrm{mol} \%$ of Zinc(II) shows the decrease in the emission signal after adding the analyte. In future work, various analytes could be applied and emission signals could be collected to find a pattern that could be used to distinguish the different explosives. 


\section{REFERENCES}

1. J-S. Yang and T.M. Swager, "Fluorescent Porous Polymer Films as TNT Chemosensors: Electronic and Structural Effects," J. Am. Chem. Soc., 1998, $120,11864-11873$.

2. J-S. Yang and T.M. Swager, "Porous Shape Persistent Fluorescent Polymer Films: An Approach to TNT Sensory Materials," J. Am. Chem. Soc., 1998, $120,5321-5322$.

3. A. Rose, C. G. Lugmair, Y.-J. Miao, J. Kim, I. A. Levitsky, V. E. Williams, and T. M. Swager, "Optimization of TNT Sensory Polymers," Proceeds. of SPIE - The International Society for Optical Engineering, 2000, 4038, (Pt. 1, Detection and Remediation Technologies for Mines and Mine like Targets), $512-518$.

4. J. V. Goodpaster and V. L. McGuffin, "Fluorescence Quenching as an Indirect Detection for Nitrated Explosives," Anal. Chem., 2001, 73, 2004 - 2011.

5. E. R. Goldman, I. L. Medintz, J. L. Whitley, A. Hayhurst, A. Clapp, H. T. Uyeda, J. R. Deschamps, M. E. Lassman, and H. Mattoussi, "A Hybrid Quantum Dot-Antibody Fragment Fluorescence Resonance Energy TransferBased TNT Sensor," J. Am. Chem. Soc., 2005, 127, 6744 - 6751.

6. S. J. Toal and W. C. Trogler, "Polymer Sensors for Nitroaromatic Explosives Detection," J. Mater. Chem., 2006, 16, 2871 - 2883.

7. I. A. Levitsky, W. B. Euler, N. Tokranova, A. Rose, "Fluorescent Polymer Porous Silicon Microcavity Devices for Explosives Detection," Appl. Phys. Lett., 2007, 90, 041904-1 - 041904-3.

8. T. Naddo, Y. Che, W. Zhang, K. Balakrishnan, X. Yang, M. Yen, J. Zhao, J. S. Moore, and L. Zang, "Detection of Explosives with a Fluorescent Nanofibril Film," J. Am. Chem. Soc., 2007, 129, 6978- 6979.

9. D. Gao, Z. Zhang, M. Wu, C. Xie, G. Guan, and D. Wang, "A Surface Functional Monomer-Directing Strategy for Highly Dense Imprinting of TNT at Surface of Silica Nanoparticles," J. Am. Chem. Soc., 2007, 129, 7859 7866.

10. D. S. Moore, "Recent Advances in Trace Explosives Detection Instrumentation,” Sens. Imaging, 2007, 8, 9 - 38.

11. A. Satrijo, S. E. Kool, and T. M. Swager, "Enhanced Luminescence from Emissive Defects in Aggregated Conjugated Polymers," Macromolecules, 
2007, 40, $8833-8841$.

12. T. L. Andrew and T. M. Swager, "A Fluorescence Turn-On Mechanism to Detect High Explosives RDX and PETN," J. Am. Chem. Soc., 2007, 129, 7254 -7255 .

13. J. C. Sanchez, A. G. DiPasquale, A. L. Rheingold, and W. C. Trogler, "Synthesis, Luminescence Properties, and Explosives Sensing with 1,1Tetraphenylsilole- and 1,1- Silafluorene-vinylene Polymers," Chem. Mater., 2007, 19, $6459-6470$.

14. J. Li, C. E. Kendig, and E. V. Nesterov, "Chemosensory Performance of Molecularly Imprinted Fluorescent Conjugated Polymer Materials," J. Am. Chem. Soc., 2007, 129, $15911-15918$.

15. S. W. Thomas III, G. D. Joly, and T. M. Swager, "Chemical Sensors Based on Amplifying Fluorescent Conjugated Polymers," Chem. Rev., 2007, 107, 1339 1386.

16. M. E. Germain and M. J. Knapp, "Turn-on Fluorescence Detection of $\mathrm{H}_{2} \mathrm{O}_{2}$ and TATP," Inorg. Chem., 2008, 47. 9748 - 9750.

17. A. Narayanan, O. P. Varnavski, T. M. Swager, and T. Goodson III, "Multiphoton Fluorescence Quenching of Conjugated Polymers for TNT Detection," J. Phys. Chem. C, 2008, 112, $881-884$.

18. M. E. Germain and M. J. Knapp, "Discrimination of Nitroaromatics and Explosives Mimics by a Fluorescent Zn(salicylaldimine) Sensor Array," J. Am. Chem. Soc., 2008, 130, 5422 - 5423.

19. S. Malashikhin and N. S. Finney, "Fluorescent Signaling Based on Sulfoxide Profluorophores: Application to the Visual Detection of the Explosive TATP," J. Am. Chem. Soc., 2008, 130, 12846 - 12847.

20. R. Tu, B. Liu, Z. Wang, D. Gao, F. Wang, Q. Fang, and Z. Zhang, “AmineCapped ZnS- Mn ${ }^{2+}$ Nanocrystals for Fluorescence Detection of Trace TNT Explosive," Anal. Chem., 2008, 80, 3458 - 3465.

21. D. Gao, Z. Wang, B. Liu, L. Ni, M. Wu, and Z. Zhang, "Resonance Energy Transfer- Amplifying Fluorescence Quenching at the Surface of Silica Nanoparticles toward Ultrasensitive Detection of TNT," Anal. Chem., 2008, $80,8545-8553$.

22. T. Naddo, X. Yang, J. S. Moore, L. Zang, "Highly Responsive Fluorescent 
Sensing of Explosives Taggant with an Organic Nanofibril Film," Sens. Actuat. B: Chem., 2008, 134, $287-291$.

23. M. S. Meaney and V. L. McGuffin, "Luminescence-based Methods for Sensing and Detection of Explosives," Anal. Bioanal. Chem., 2008, 391, 2557 -2576 .

24. G. He, G. Zhang, F. Lu, and Y. Fang, "Fluorescent Film Sensor for VaporPhase Nitroaromatic Explosives via Monolayer Assembly of Oligo(diphenylsilane) on Glass Plate Surfaces," Chem. Mater., 2009, 21, 1494 $-1499$.

25. Q. Fang, J. Geng, B. Liu, D. Gao, F. Li, Z. Wang, G. Guan, and Z. Zhang, "Inverted Opal Fluorescence Film Chemosensor for the Detection of Explosive Nitroaromatic Vapors through Fluorescence Resonance Energy Transfer," Chem. Eur. J., 2009, 15, $11507-11514$.

26. K. Shiraishi, T. Sanji, and M. Tanaka, "Trace Detection of Explosive Particulates with a Phosphole Oxide," Appl. Mater. Interfaces, 2009, 1, 1379 1382.

27. A. Qin, J. W. Y. Lam, L. Tang, C. K. W. Jim, H. Zhao, J. Sun, and B. Z. Tang, "Polytriazoles with Aggregation-Induced Emission Characteristics: Synthesis by Click Polymerization and Application as Explosive Chemosensors," Macromolecules, 2009, 42, 1421 - 1424.

28. W. Z. Yuan, H. Zhao, X. Y. Shen, F. Mahtab, J. W. Y. Lam, J. Z. Sun, and B. Z. Tang, "Luminogenic Polyacetylenes and Conjugated Polyelectrolytes: Synthesis, Hybridization with Carbon Nanotubes, Aggregation-Induced Emission, Superamplification in Emission Quenching by Explosives, and Fluorescent Assay for Protein Quantitation," Macromolecules, 2009, 42, 9400 -9411 .

29. H. H. Nguyen, X. Li, N. Wang, Z. Y. Wang, J. Ma, W. J. Bock, and D. Ma, "Fiber-Optic Detection of Explosives Using Readily Available Fluorescent Polymers," Macromolecules, 2009, 42, 921 - 926.

30. Y. Long, H. Chen, Y. Yang, H. Wang, Y. Yang, N. Li, K. Li, J. Pei, and F. Liu, "Electrospun Nanofibrous Film Doped with a Conjugated Polymer for DNT Fluorescence Sensor," Macromolecules, 2009, 42, 6501 - 6509.

31. J. Liu, Y. Zhong, J. W. Y. Lam, P. Lu, Y. Hong, Y. Yu, Y. Yue, M. Faisal, H. H. Y. Sung, I. D. Williams, K. S. Wong, and B. Z. Tang, "Hyperbranched Conjugated Polysiloles: Synthesis, Structure, Aggregation-Enhanced Emission, Multicolor Fluorescent Photopatterning, and Superamplified Detection of 
Explosives," Macromolecules, 2010, 43, 4921 - 4936.

32. W. Chen, N. B. Zuckerman, J. P. Konopleski, and S. Chen, "PyreneFunctionalized Ruthenium Nanoparticles as Effective Chemosensors for Nitroaromatic Derivatives," Anal. Chem., 2010, 82, 461 - 465.

33. Y. Chen, Z. Chen, Y. He, H. Lin, P. Sheng, C. Liu, S. Luo, and Q. Cai, "LCysteine-capped CdTe QD-based Sensor for Simple and Selective Detection of Trinitrotoluene," Nanotechnology, 2010, 21, 125502/1 - 125502/5.

34. Y. Che, X. Yang, G. Liu, C. Yu, H. Ji, J. Zuo, J. Zhao, and L. Zang, "Ultrathin n-Type Organic Nanoribbons with High Photoconductivity and Application in Optoelectronic Vapors Sensing of Explosives," J. Am. Chem. Soc., 2010, 132, $5743-5750$.

35. R. C. Stringer, S. Gangopadhyay, and S. A. Grant, "Detection of Nitroaromatic Explosives Using a Fluorescent-Labeled Imprinted Polymer," Anal. Chem., 2010, $82,4015-4019$.

36. A. Ponnu and E. V. Anslyn, "A Fluorescence-Based Cyclodextrin Sensor to Detect Nitroaromatic Explosives," Supramol. Chem., 2010, 22, 65- 71.

37. M. Woodka and V. P. Schnee, "Fluorescent Polymer Sensor Array for Detection and Discrimination of Explosives in Water," Anal. Chem., 2010, 82, $9917-9924$.

38. H. Cavaye, P. E. Shaw, X. Wang, P. L. Burn, S.-C. Lo, and P. Meredith, "Effect of Dimensionality in Dendrimeric and Polymeric Fluorescent Materials for Detecting Explosives," Macromolecules, 2010, 43, 10253 - 10261.

39. G. He, N. Yan, J. Yang, H. Wang, L. Ding, S. Yin, and Y. Fang, "PyreneContaining Conjugated Polymer-Based Fluorescent Films for Highly Sensitive and Selective Sensing of TNT in Aqueous Medium," Macromolecules, 2011, $44,4759-4766$.

40. B. Xu, X. Wu, H. Li, H. Tong, and L. Wang, "Selective Detection of TNT and Picric Acid by Conjugated Polymer Film Sensors with Donor-Acceptor Architecture," Macromolecules, 2011, 44, 5089 - 5092.

41. K. Zhang, H. Zhou, Q. Mei, S. Wang, G. Guan, R. Liu, J. Zhang, and Z. Zhang, "Instant Visual Detection of Trinitrotoluene Particulates on Various Surfaces by Ratiometric Fluorescence of Dual-Emission Quantum Dots Hybrid," J. Am. Chem. Soc., 2011, 133, 8424 - 8427.

42. D. A. Olley, E. J. Wren, G. Vamvounis, M. J. Fernee, X. Wang, P. L. Burn, P. 
Meredith, and P. E. Shaw, "Explosive Sensing with Fluorescent Dendrimers: The Role of Collisional Quenching," Chem. Mater., 2011, 23, 789 - 794.

43. Y. Peng, A.-J. Zhang, M. Dong, Y.-W. Wang, "A Colorimetric and Fluorescent Chemosensor for the Detection of an Explosive - 2,4,6Trinitrophenol (TNP)," Chem. Commun., 2011, 47, 4505 - 4507.

44. J. Wang, L. Yang, S. Boriskina, B. Yan, and B. M. Reinhard, "Spectroscopic Ultra-Trace Detection of Nitroaromatic Gas Vapor on Rationally Designed Two-Dimensional Nanoparticle Cluster Arrays," Anal. Chem., 2011, 83, 2243 -2249 .

45. W.-S. Zou, D. Sheng, X. Ge, J.-Q. Qiao, and H.-Z. Lian, "Room-Temperature Phosphorescence Chemosensor and Rayleigh Scattering Chemodosimeter Dual-Recognition Probe for 2,4,6-Trinitrotoluene Based on Manganese-Doped ZnS Quantum Dots," Anal. Chem., 2011, 83, 30 - 37.

46. Y. Xia, L. Song, and C. Zhu, "Turn-On and Near-Infrared Fluorescent Sensing for 2,4,6- Trinitrotoluene Based on Hybrid (Gold Nanorod)-(Quantum Dots) Assembly," Anal. Chem., 2011, 83, 1401 - 1407.

47. Y. Wang, B. R. Rae, R. K. Henderson, Z. Gong, J. Mckendry, E. Gu, M. D. Dawson, G. A. Turnbull, and I. D. W. Samuel, "Ultra-portable Explosives Sensor Based on a CMOS Fluorescence Lifetime Analysis Microsystem," AIP Advances, 2011, 1, 032115/1 - 032115/10.

48. M. A. Matoian, R. Sweetman, E. C. Hall, S. Albanese, W. B. Euler, "Light Trapping to Amplify Metal Enhanced Fluorescence with Application for TNT Sensing," J. Fluoresc., 2013, 23, $877-880$.

49. C. A. Latendresse, S.C. Fernandes, S. You, H. Q. Zhang, W. B. Euler, "A Fluorometric Array for the Detection of Military Explosives and IED Materials," Anal. Methods, 2013, 5, 5457 - 5463.

50. S. Rochat and T. M. Swager, "Fluorescence Sensing of Amine Vapors Using a Cationic Conjugated Polymer Combined with Various Anions," Ang. Chem. Int. Ed., 2014, 53, $9792-9796$.

51. S. Yang, C. Wang, C. Liu, Y. Wang, Y. Xiao, J. Li, Y. Li, and R. Yang, "Fluorescence Modulation by Absorbent on Solid Surface: An Improved Approach for Designing Fluorescent Sensor," Anal. Chem., 2014, 86, 7931 7938.

52. W. Xu, C. Ren, C. L. Teoh, J. Peng, S. H. Gadre, H.-W. Rhee, C.-L. K. Lee, and Y.-T. Chang, "An Artificial Tongue Fluorescent Sensor Array for 
Identification and Quantitation of Various Heavy Metal Ions," Anal. Chem., 2014, $86,8763-8769$.

53. S. N. A. Jenie, S. Pace, B. Sciacca, R. D. Brooks, S. E. Plush, and N. H. Voelcker, "Lanthanide Luminescence Enhancements in Porous Silicon Resonant Microcavities," ACS Appl. Mater. Interfaces, 2014, 6, 12012 12021.

54. S. Wang, L. Ding, J. Fan, Z. Wang, and Y. Fang, "Bispyrene/SurfactantAssembly-Based Fluorescent Sensor Array for Discriminating Lanthanide Ions in Aqueous Solution," ACS Appl. Mater. Interfaces, 2014, 6, 16156 - 16165.

55. J. W. Nugent, H. Lee, H.-S. Lee, J. H. Reibenspies, and R. D. Hancock, “The Effect of $\pi$ Contacts between Metal Ions and Fluorophores on the Fluorescence of PET Sensors: Implications for Sensor Design for Cations and Anions," Inorg. Chem., 2014, 53, $9014-9026$.

56. B. D. de Greñu, D. Moreno, T. Torroba, A. Berg, J. Gunnars, T. Nilsson, R. Nyman, M. Persson, J. Pettersson, I. Eklind, and P. Wästerby, "Fluorescent Discrimination between Traces of Chemical Warfare Agents and Their Mimics," J. Am. Chem. Soc., 2014, 136, 4125-4128.

57. Q. Zhou, T. M: Swager, J. Am. Chem. Soc., 1995, 117, 7017-7018.

58. C. J. Cumming, C. Aker, M. Fisher, M. Fox, M. J. la Grone, D. Reust, M. G. Rockley, T. M. Swager, E. Towers, V. Williams, IEEE Transactions on Geoscience and Remote Sensing, 2001, 39, 1119-1128.

59. S. J. Toal, W. C. Trogler, J. Mater. Chem., 2006, 16, 2871-2883.

60. The Role of Solution Phase Water on the Deposition of Thin Films of Poly(vinylidene fluoride)," M. Benz, W. B. Euler, O. J. Gregory, Macromolecules, 2002, 35, 2682-2688.

61. F.P. Schafer, “ Topics in Applied Physics,” Vol. 1 (Springer, Berlin, 1973).

62. M. Benz and W. B. Euler, "Determination of the Crystalline Phases of Poly(vinylidene fluoride) Under Different Preparation Conditions Using Differential Scanning Calorimetry and Infrared Spectroscopy," J. Appl. Polym. Sci., 2003, 89, 1093-1100. 


\section{APPENDICES}

Appendix A: IR Spectrum of $\mathrm{Fe}\left(a c a c_{3}\right)$ and Grignard Reagent.

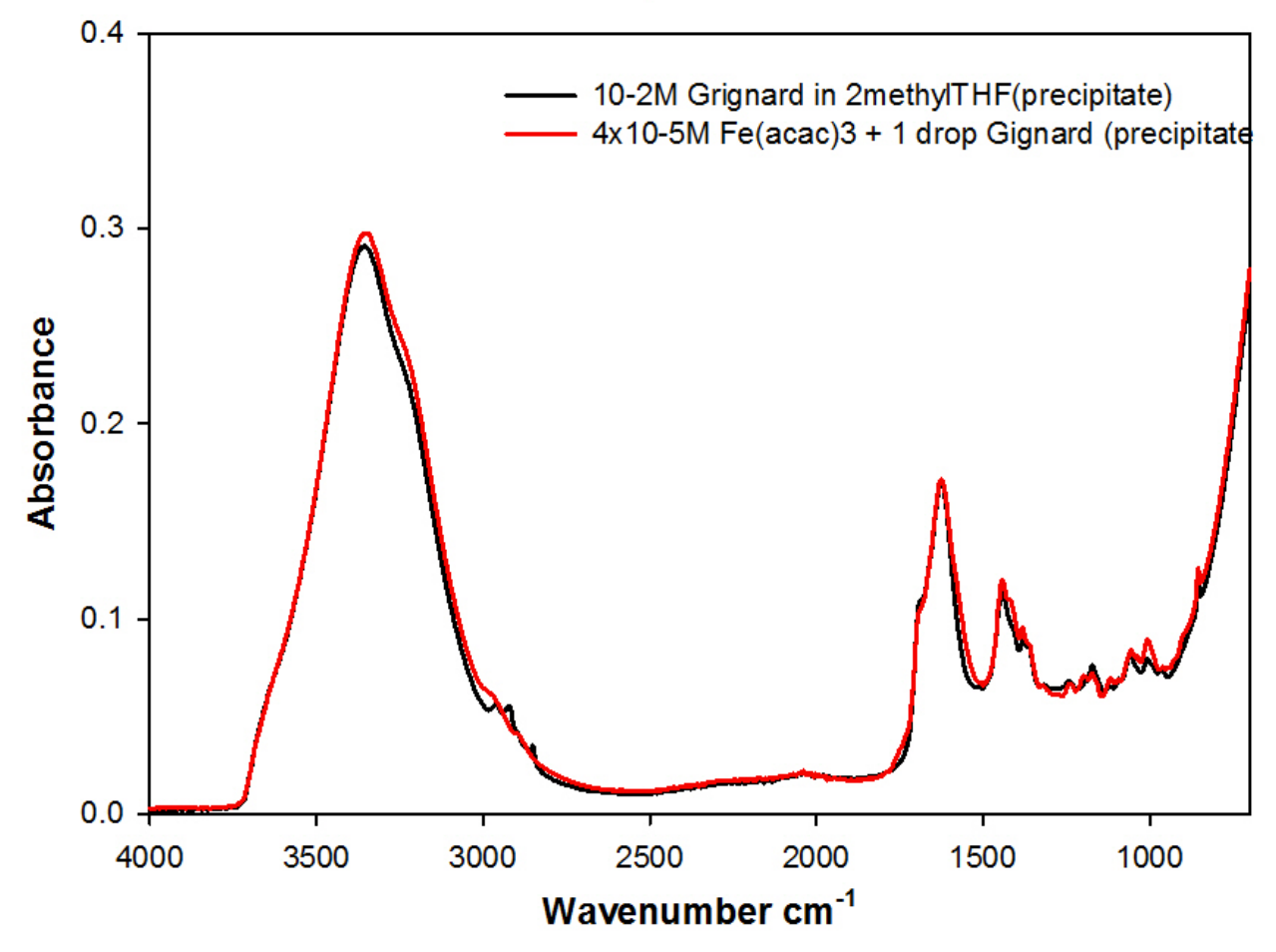


Appendix B: IR Spectrum of $\mathrm{Fe}\left(a c a c_{2}\right)$ and $\mathrm{Fe}\left(a c a c_{3}\right)$.

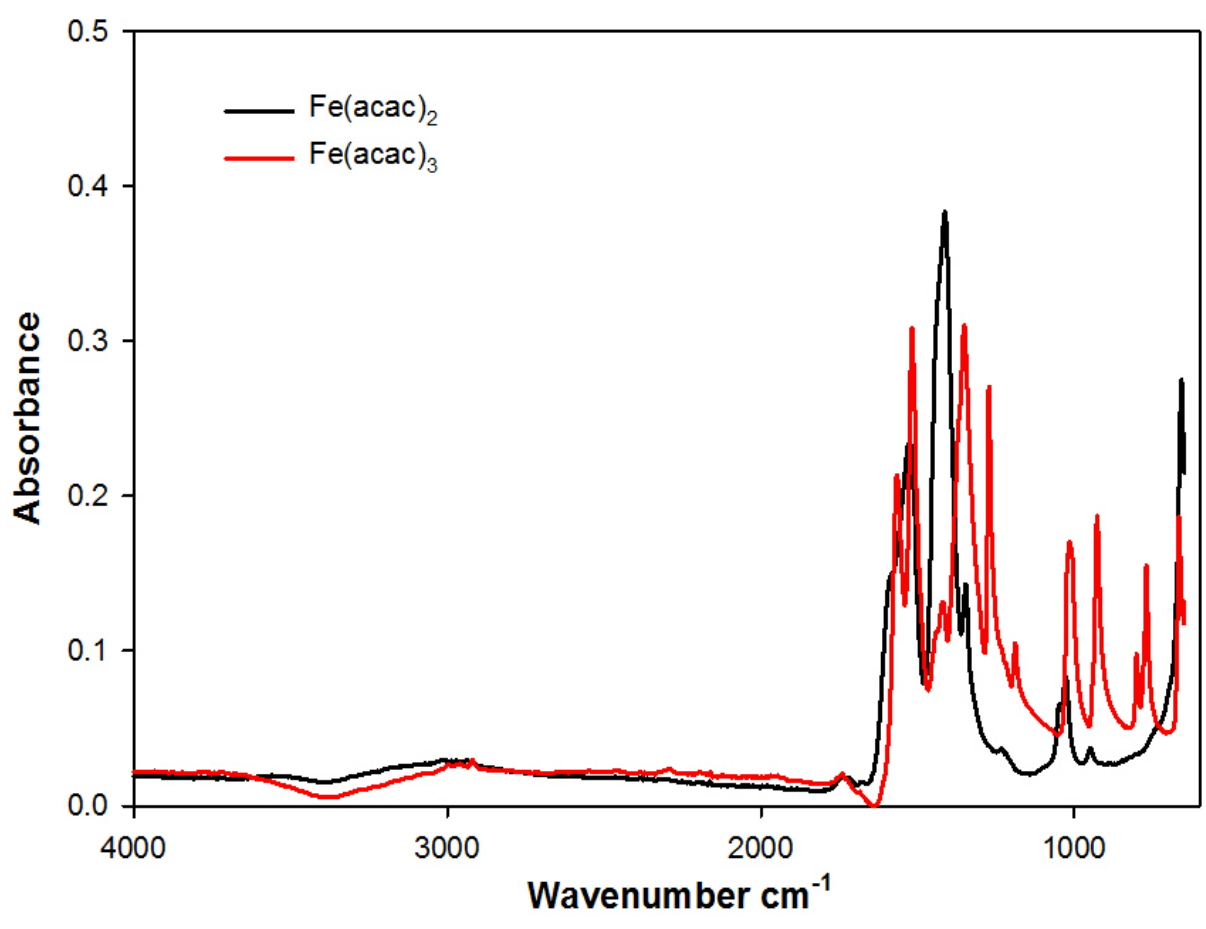

Appendix C: IR Spectrum of $\mathrm{Fe}\left(a c a c_{3}\right)$, bpy Ligand and Grignard Reagent.

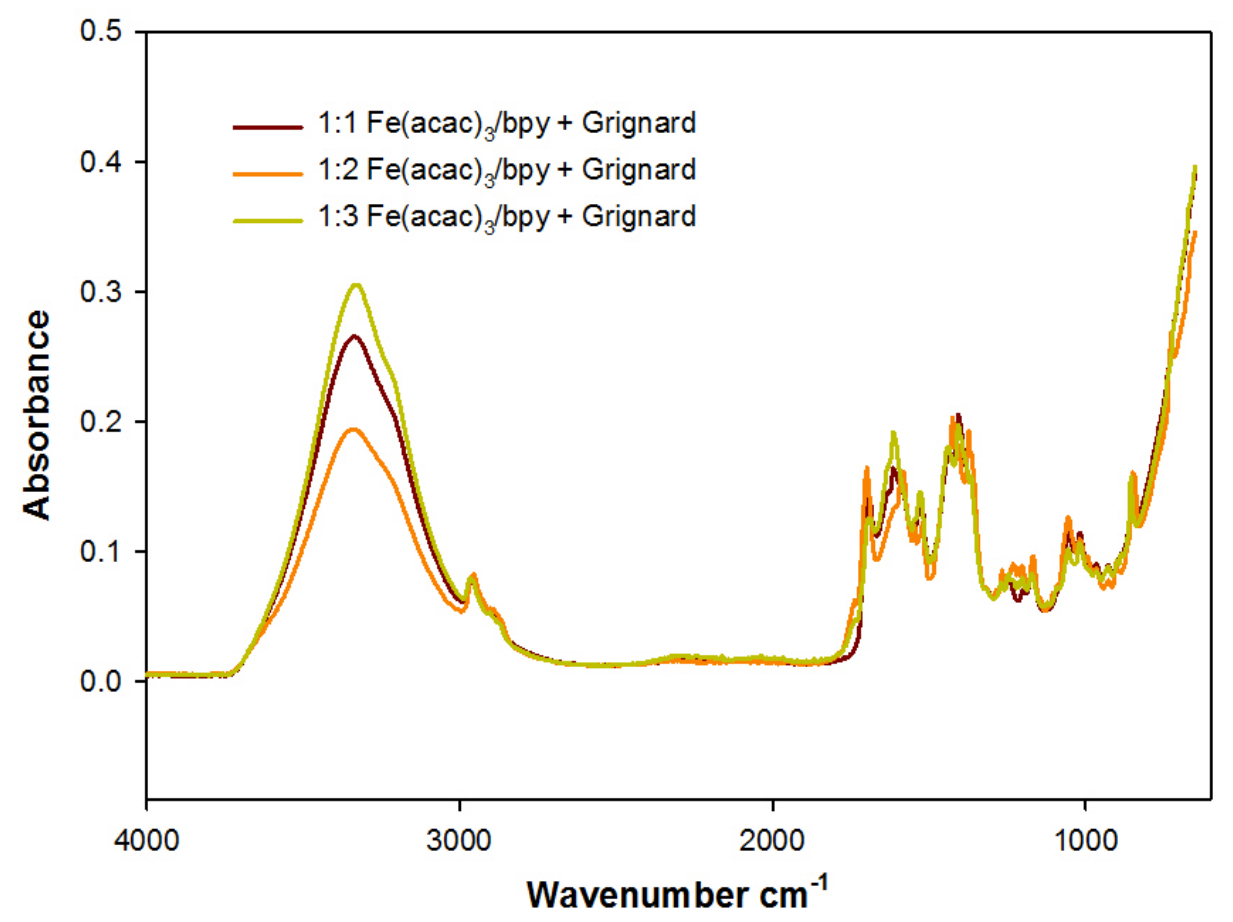


Appendix D: Absorbance Spectra of $\mathrm{Fe}\left(a c a c_{3}\right)$ and bpy Ligand.

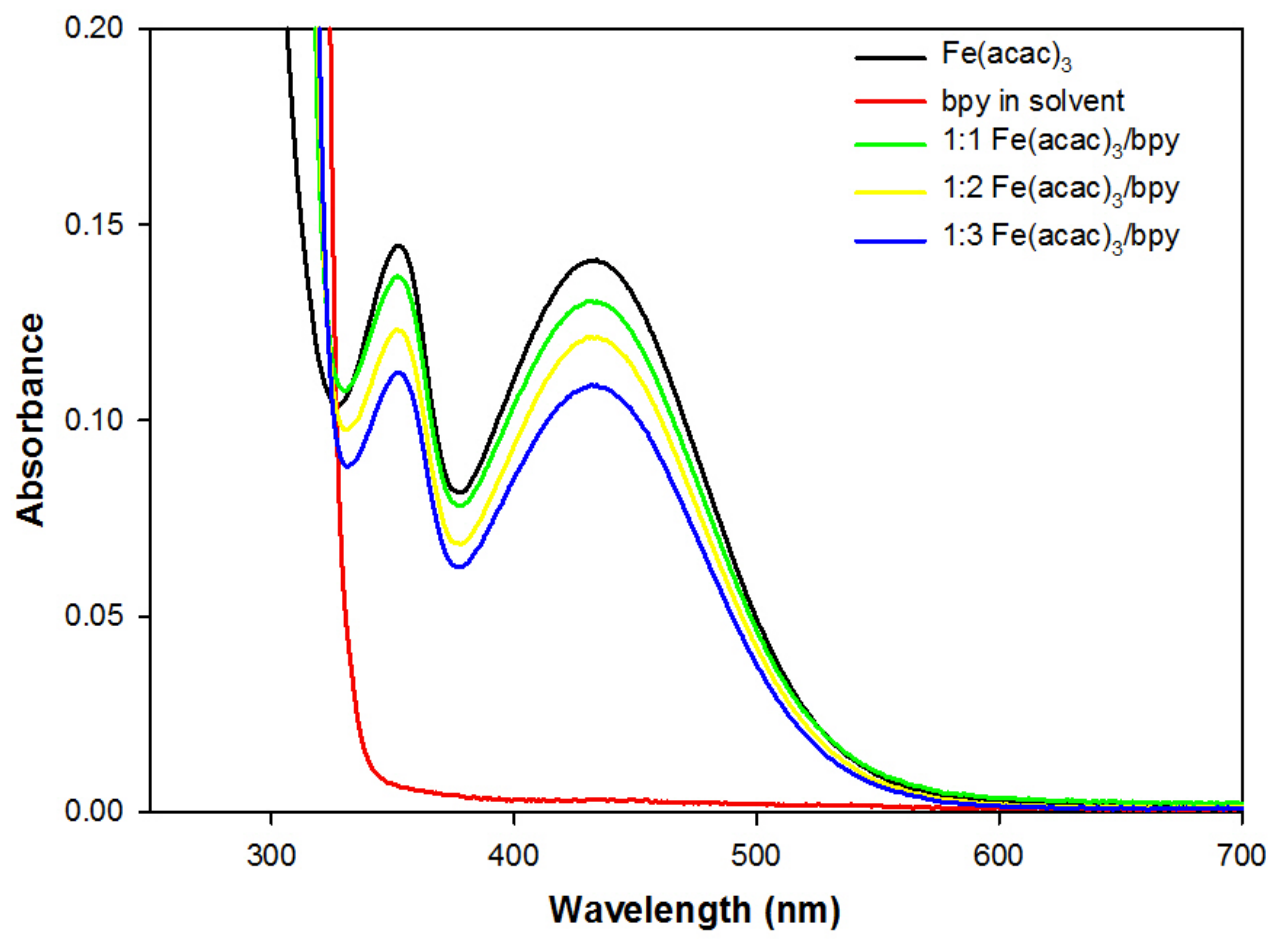


Appendix E: Absorbance Spectra of Fe $\left(\mathrm{acac}_{3}\right)$ and Phenanthroline Ligand.

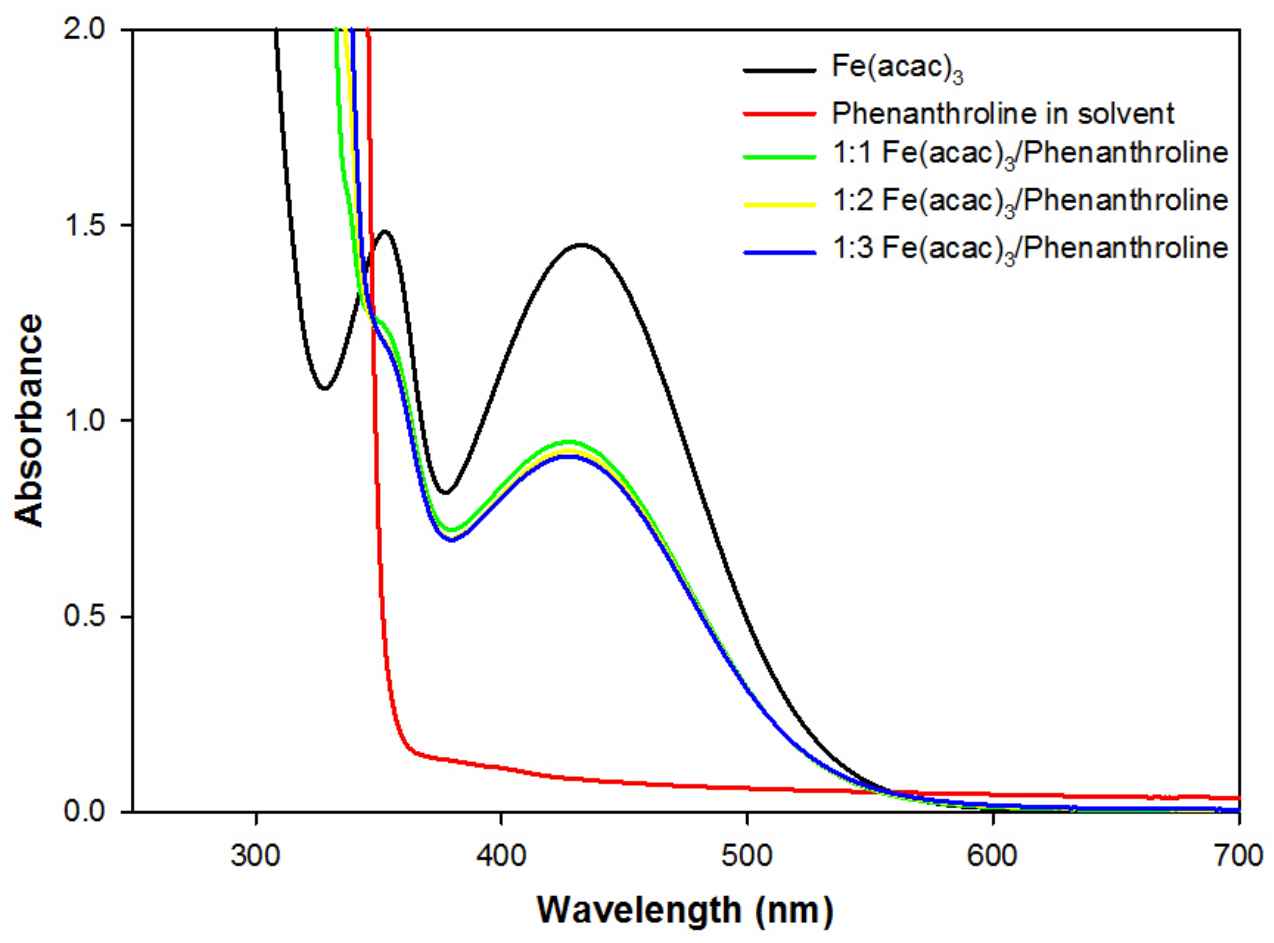

Appendix F: Absorbance Spectra of $\mathrm{Fe}\left(a c a c_{3}\right)$ and dtbpy Ligand.

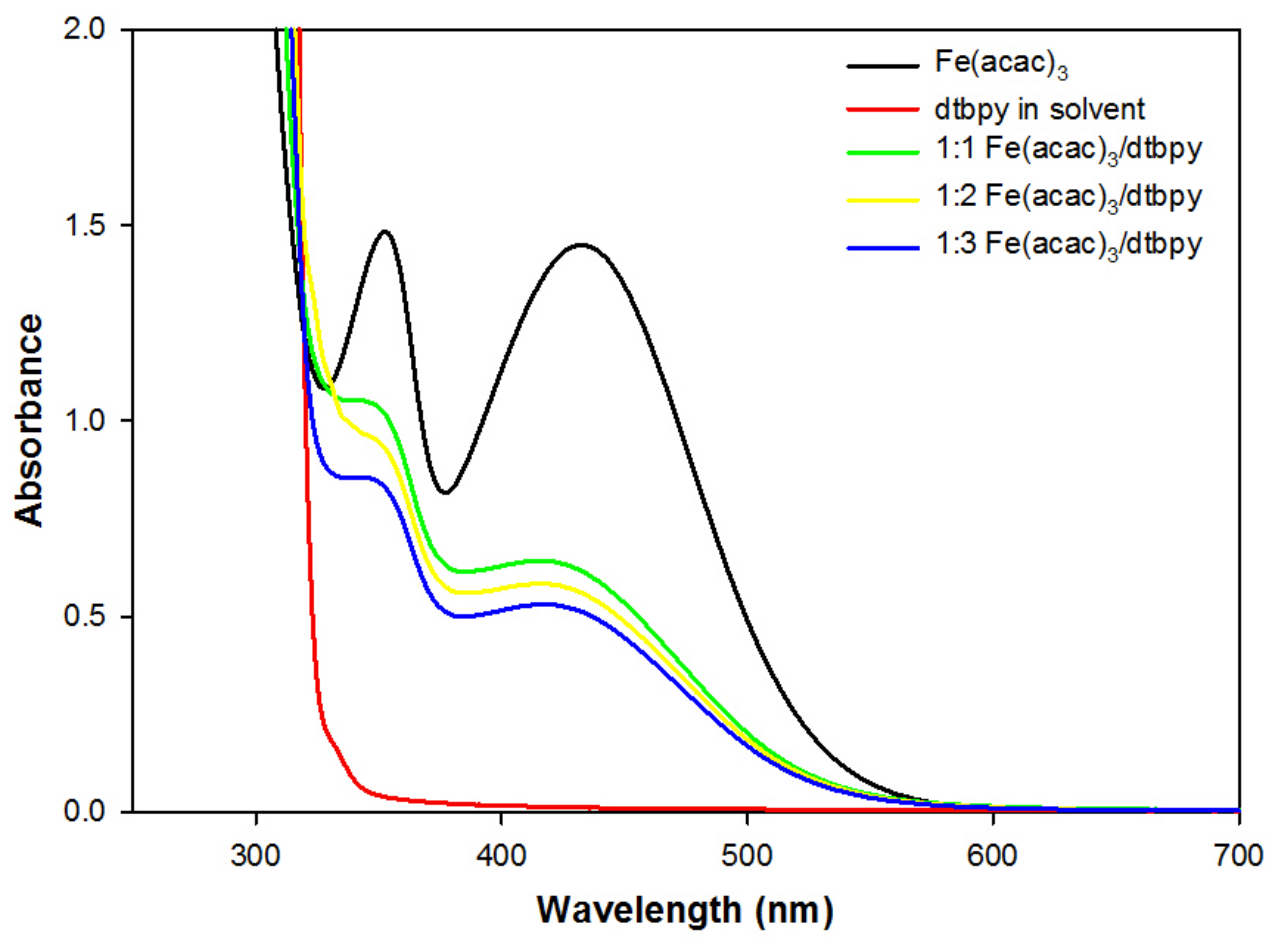


Appendix G: Absorbance Spectra of $\mathrm{Fe}\left(a c a c_{3}\right)$ and Grignard Reagent Over Time.

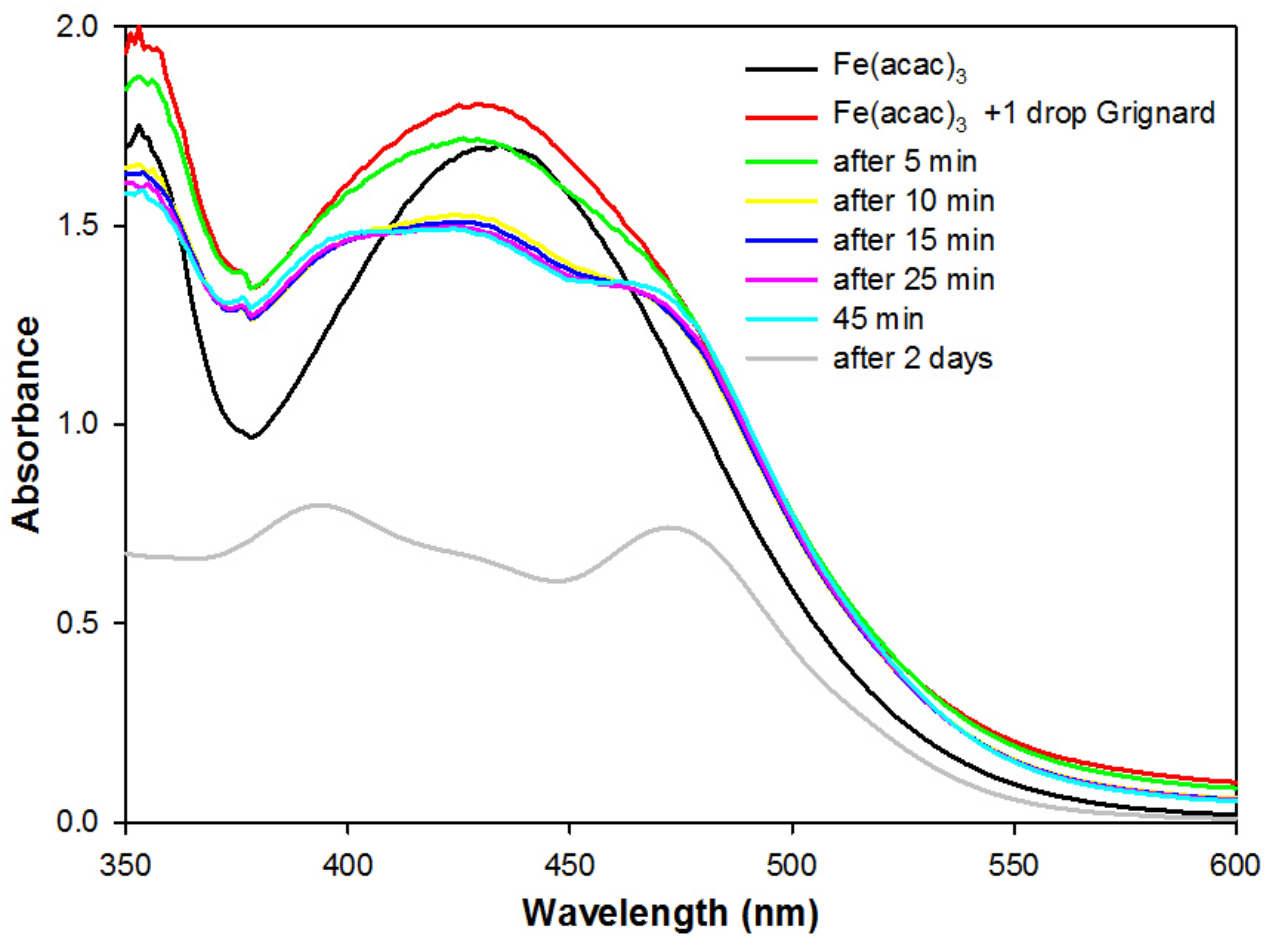


Appendix H: Absorbance Spectra of $\mathrm{Fe}\left(\mathrm{acac}_{3}\right) / \mathrm{bpy}$ at 1:1 Ratio and Grignard Reagent Over Time.

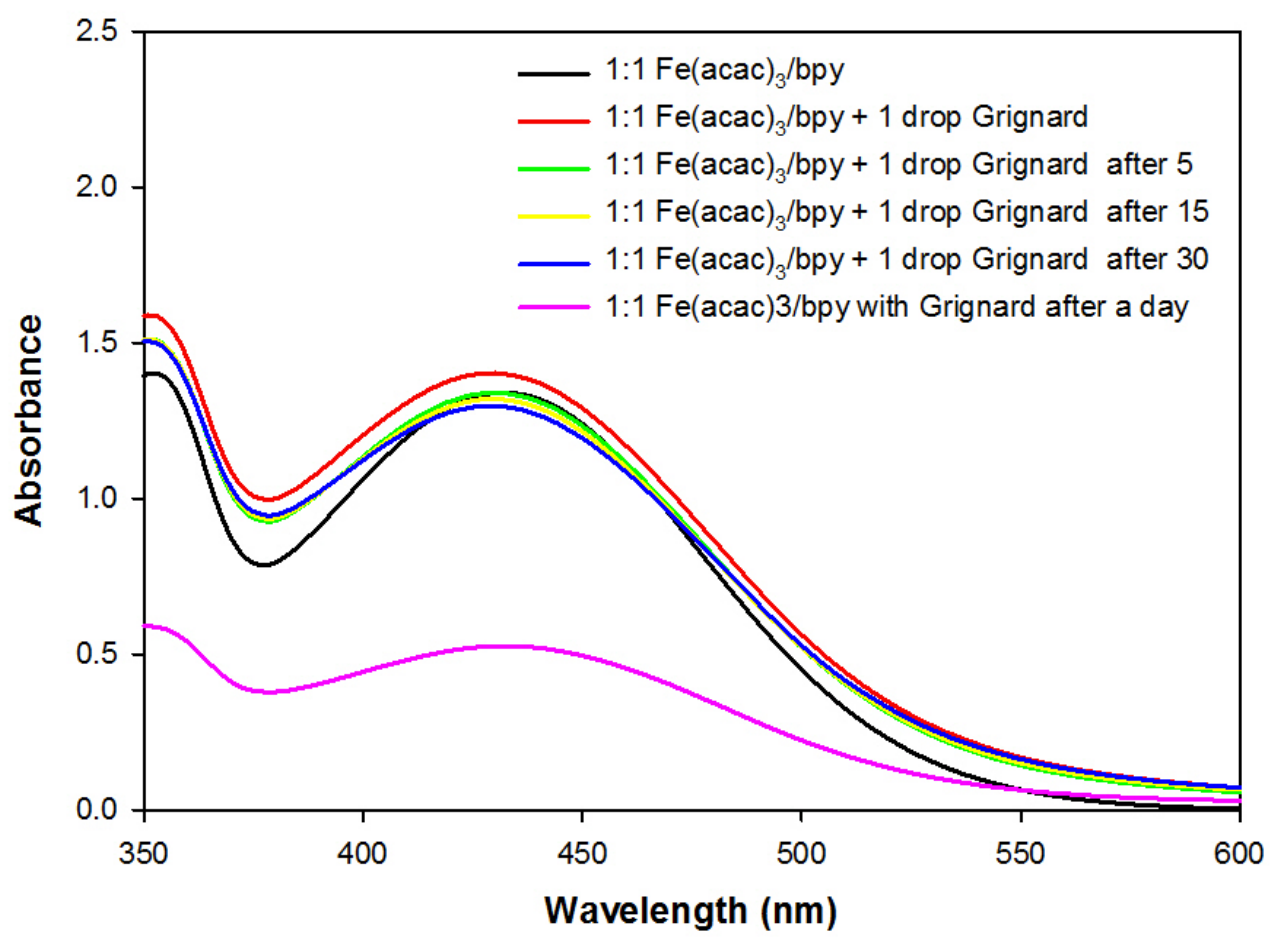

Appendix I: Absorbance Spectra of $\mathrm{Fe}\left(\mathrm{acac}_{3}\right) / \mathrm{bpy}$ at 1:2 Ratio and Grignard Reagent Over Time.

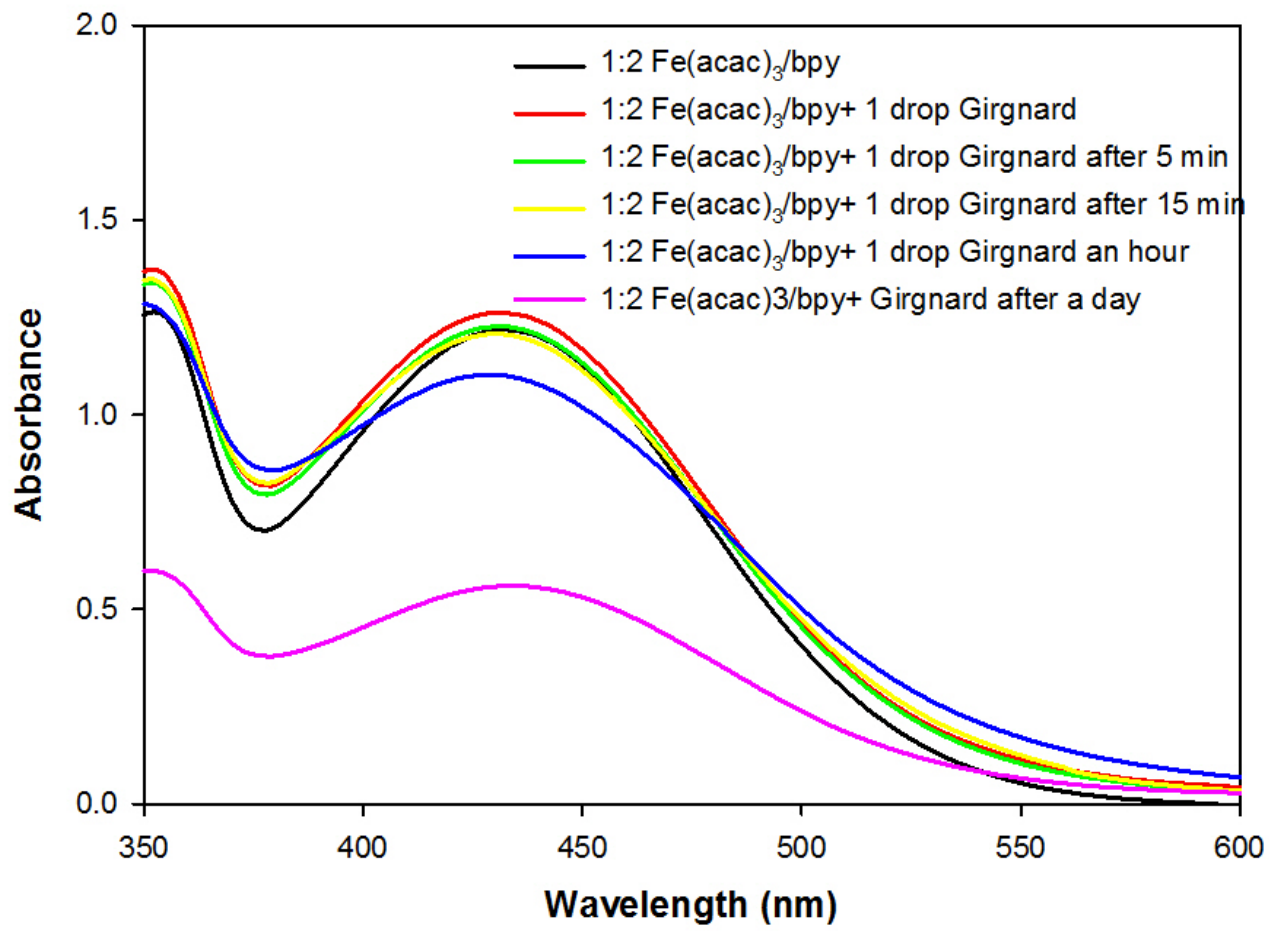


Appendix J: Absorbance Spectra of $\mathrm{Fe}\left(\mathrm{acac}_{3}\right) / \mathrm{bpy}$ at 1:3 Ratio and Grignard Reagent Over Time.

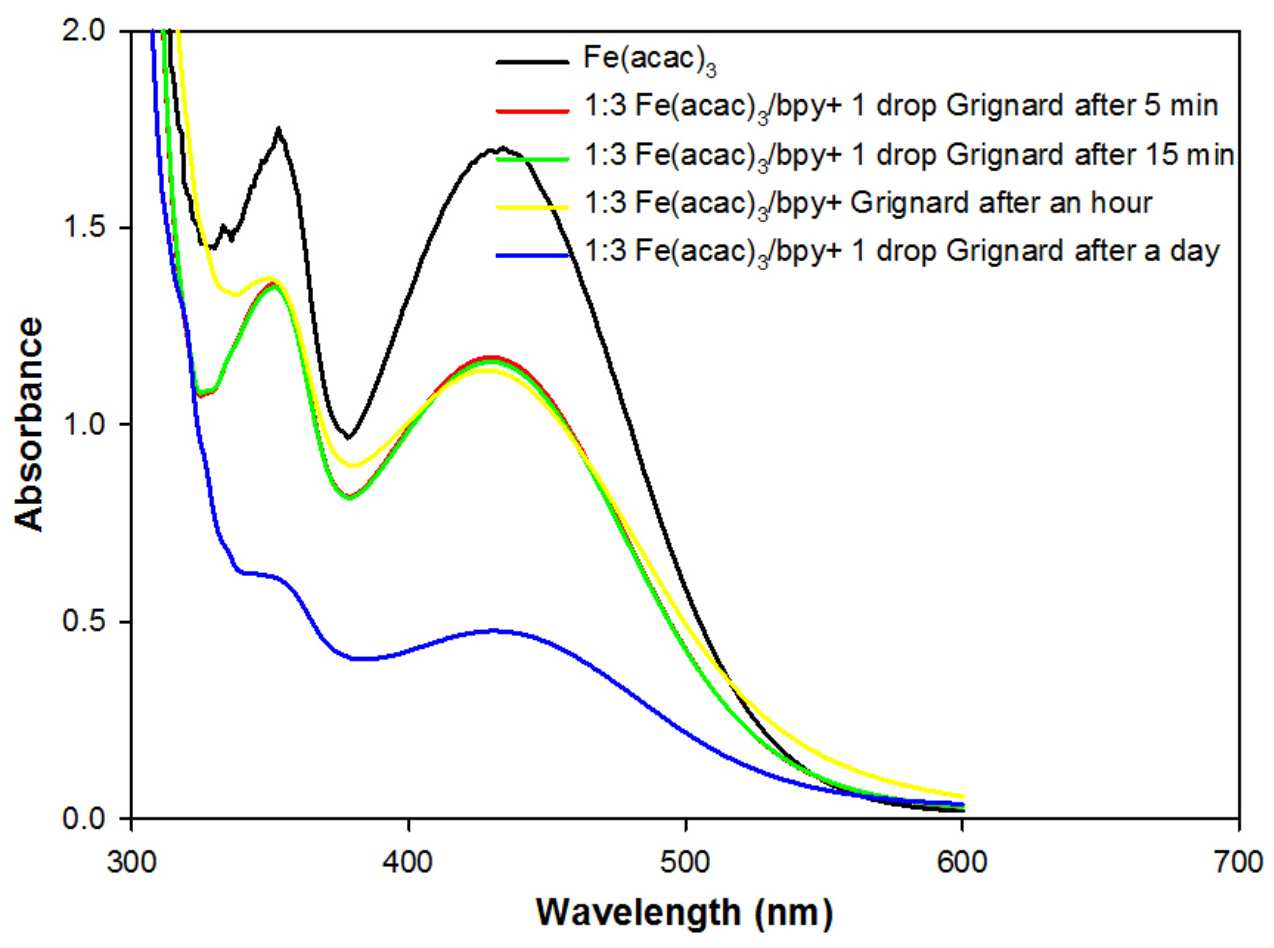


Appendix K: Absorbance Spectra of $\mathrm{Fe}\left(\mathrm{acac}_{3}\right) \mathrm{Vs}$. Fe( $\left(\mathrm{acac}_{3}\right) / \mathrm{bpy}$ and Grignard Reagent.

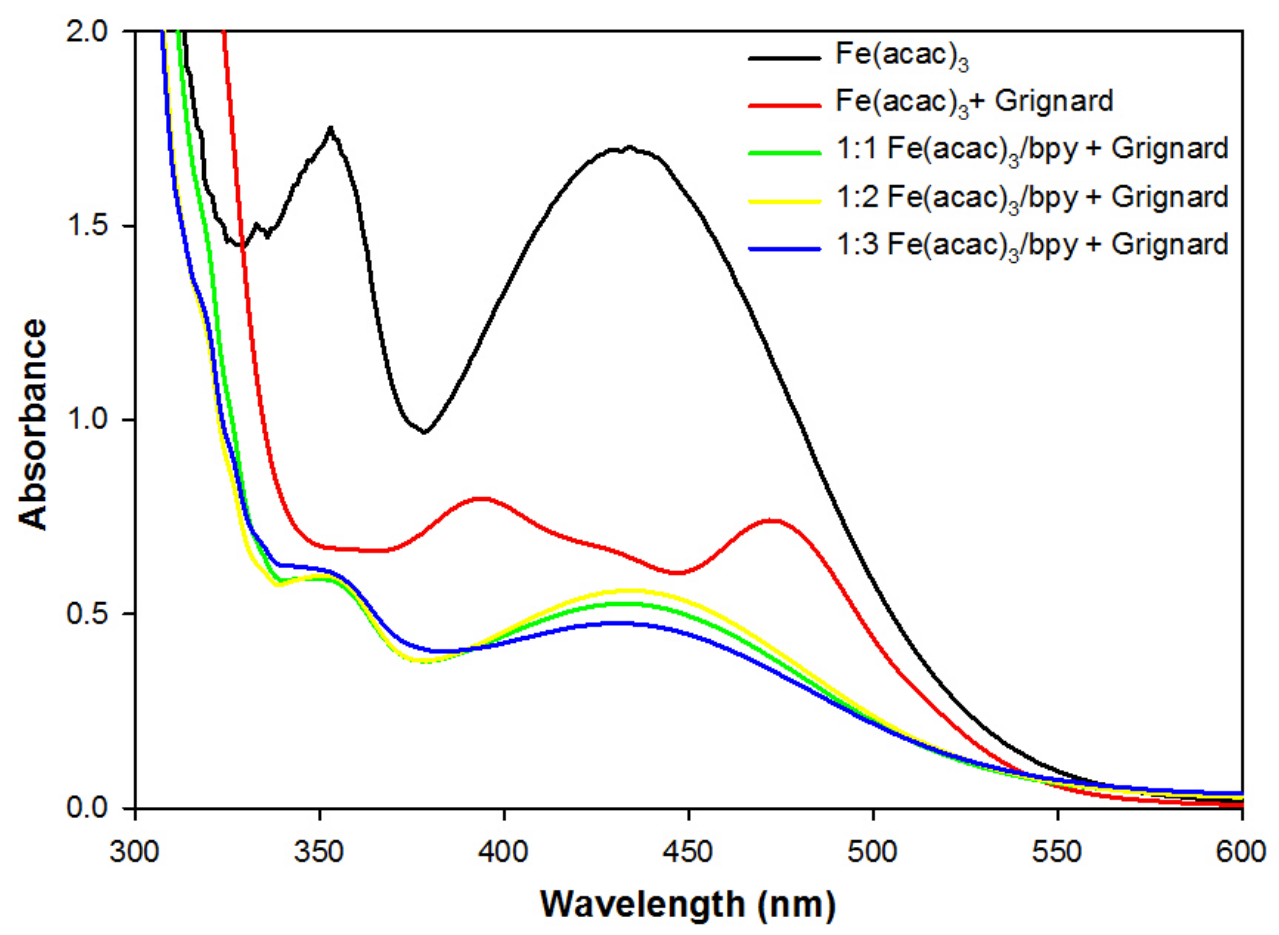

Appendix L: Reflection Spectrum of $\mathrm{Fe}\left(\mathrm{acac}_{3}\right) / \mathrm{bpy}$ and Grignard.

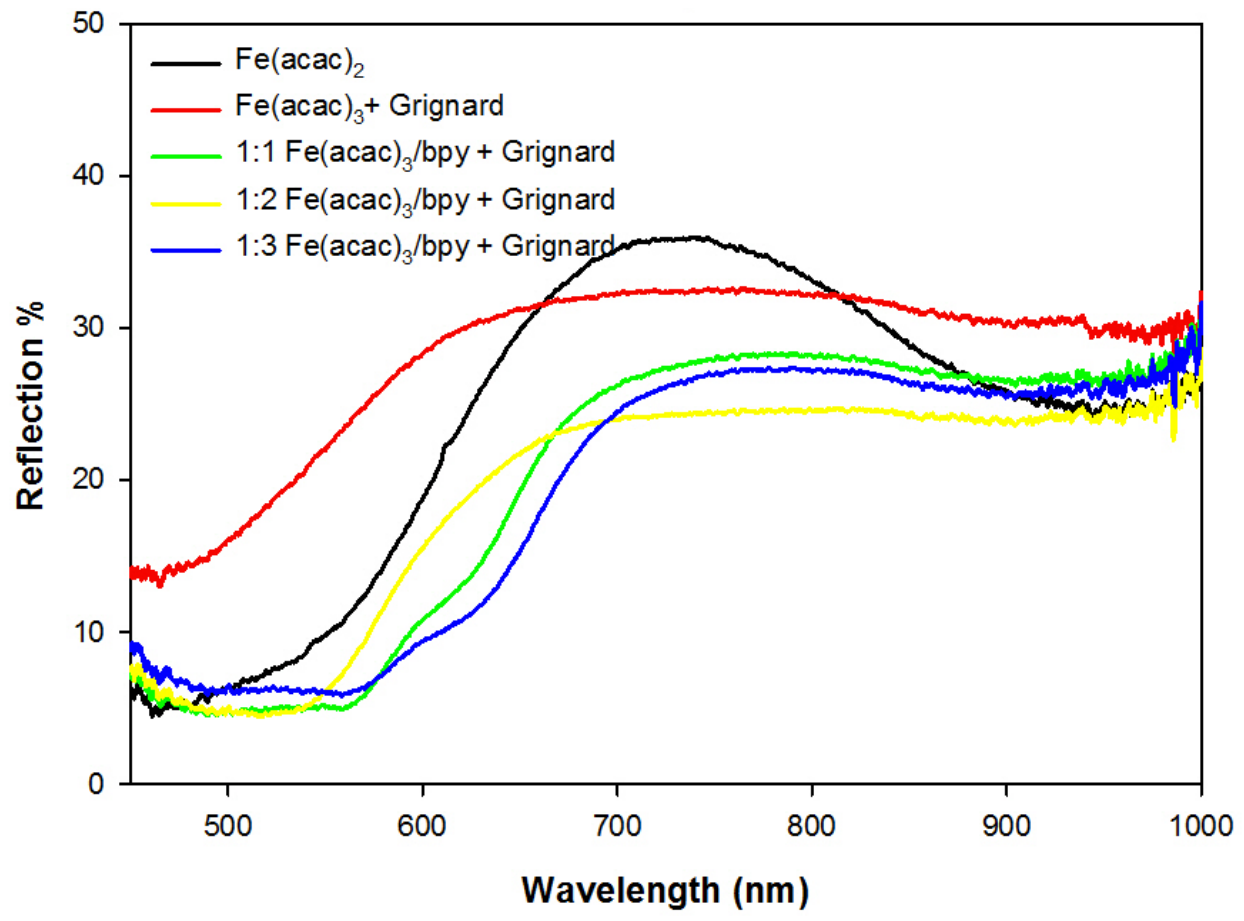

